\title{
AD-A261 728
}

PL-TR-92-2296

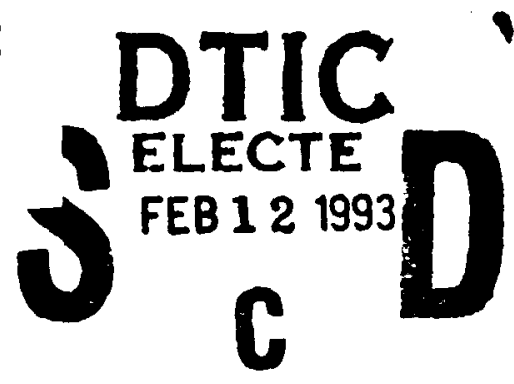

\section{PROGRESS IN IMPULSE APPROACH RESEARCH}

H. Dothe

J. M. Sindoni

Yap Analytics, Inc.

594 Marrett Road

Lexington, MA 02173

30 October 1992

Scientific Report No. 1

Approved for public release; distribution unlimited

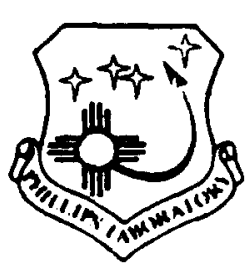

PHILLIPS LABORATORY

Directorate of Geophysics

AIR FORCE MATERIEL COMMAND

HANSCOM AIR FORCE BASE, MA 01731-5000

$982 ?$ 
This technical report has been reviewed and is approved for publication.

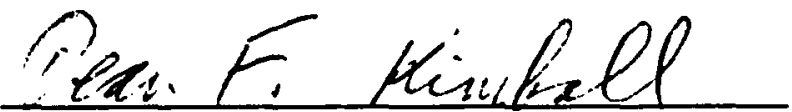

DEAN F. KIMBALL Contract Manager Simulation Branch

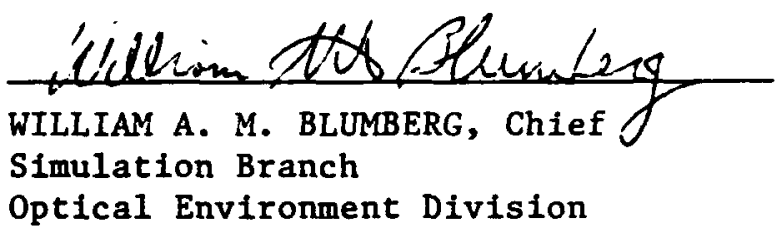

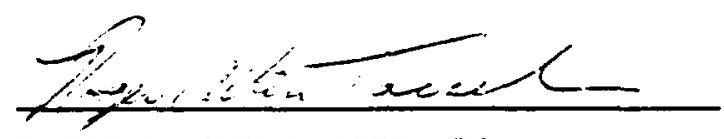

ROGER A. VAN TASSEL, Director

Optical Environment Division

This document has been reviewed by the ESC Public Affairs Office (PA) and is releasable to the National Technical Information Service (NTIS).

Qualified requestors may obtain additional copies from the Defense Technical Information Center. All others should apply to the National Technical

Information Service.

If your address has changed, or if you wish to be removed from the mailing 11st, or if the addressee is no longer employed by your organization, please notify PL/TSI, Hanscom AFB, MA 01731-5000. This will assist us in maintaining a current mailing list.

Do not return coples of this report unless contractual obligations or notices on a specific document requires that it be returned. 
Publle reponting burden for this collection of information is estimated to average 1 hour per response including the time for reviewing instructions. searching existing oata soufces

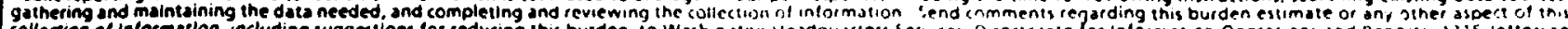
Collection ort mer

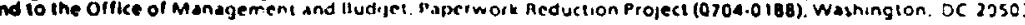

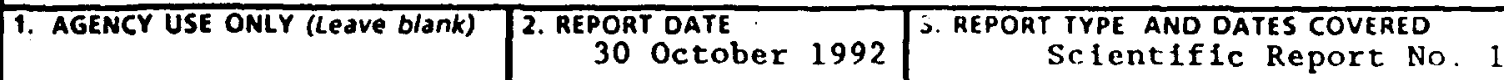

\section{TITLE AND SUBTITLE}

Progress in Impulse Approach Research 5. FUNDING NUMBERS

\section{PE $63215 \mathrm{C}$}

PR S 322

TA 03

WU AP

Contract No

H, Dothe

J.M. Sindoni

7. PERFORMING ORGANIZATION NAME(S) AND ADORESS(ES)

Yap Analytics, Inc.

594 Marrett Road

Lexington, MA 02173

F19628-91-C-0142

8. PERFORMING ORGANIZATION REPORT NUMBER

YAI - TR - 107

9. SPONSORING/MONITORING AGENCY NAME(S) AND ADDRESS(ES)

Phillips Laboratory

Hanscom AFB, MA 01731-5000

Contract Manager : Dean Kimball/GPOS

11. SUPPLEMENTARY NOTES

\begin{tabular}{|l|l|}
\hline 12a. DISTRIBUTION/AVAILABILITY STATEMENT & 12b. DISTRIBUTION CODE \\
Approved for public release; \\
distribution unlimited
\end{tabular}

\section{ABSTRACT (Maximum 200 words)}

This report entails the progress made during the first contract year in the research of Impulse Approach (IA) modeling. The IA technique was successfully applied to the Ar-CsF scattering system with results that compared favorably with observations. Limitations and discrepancies were also found in the treatment of other scattering systems which were attributed to the confinement of the IA method to the single collision $T$ matrix. Work is at present progres sing on extending the approach to the double collision $T$ Matrix. The Distorted Wave Impulse Approach (DWIA) is also being developed to model observed distributions of rotational transitional probabilities of the $\mathrm{Li}^{+}-\mathrm{N}_{2}$ scattering system.

\begin{tabular}{|c|c|c|c|}
\hline $\begin{array}{l}\text { 14. SUEJEC IERMS } \\
\text { BaII tic Collision } \\
\text { Impulse Approach }\end{array}$ & \multicolumn{2}{|c|}{$\begin{array}{l}\text { Three-Body } T \text { Matrix } \\
\text { Distorted Wave Impulse Approach }\end{array}$} & $\begin{array}{l}\text { 15. NUMBEA OF PAGES } \\
108 \\
\text { 16. PRICE CODE }\end{array}$ \\
\hline $\begin{array}{l}\text { 17. SECURITYCLASSIFICATION } \\
\text { Of REPORT } \\
\text { Unclassifled }\end{array}$ & $\begin{array}{l}\text { 18. SECUATYY CLASSIFICATION } \\
\text { OF THIS PAGE } \\
\text { Unclass ifled }\end{array}$ & $\begin{array}{l}\text { 19. SECUAITY CLASSIFICATION } \\
\text { OF ADSYRACT } \\
\text { Unclassifled }\end{array}$ & $\begin{array}{l}\text { 20. LIMITATION OF ABSTRACT } \\
\text { SAR }\end{array}$ \\
\hline
\end{tabular}


THIS PAGE INTENTIONALLY

LEFT BLANK

ii 
TABLE OF CONTENTS

SECTION

PAGE

TABLE of CONTENTS ..................... i i

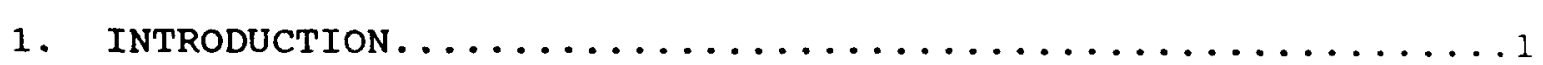

2. DOUBLE COLLISION T MATRIX...................

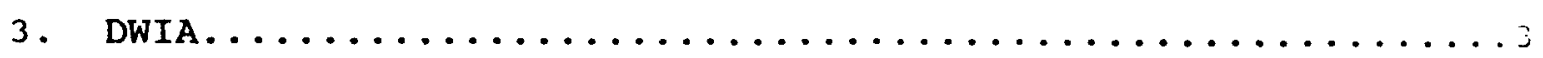

4. FUTURE WORK..........................

REFERENCES..........................

APPENDIX A

INELASTIC AND BALLISTIC PROCESSES RESULTING FROM

CsF-Ar COLLISIONS ......................

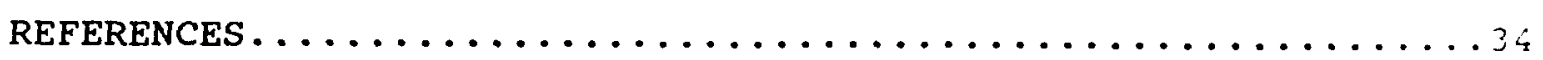

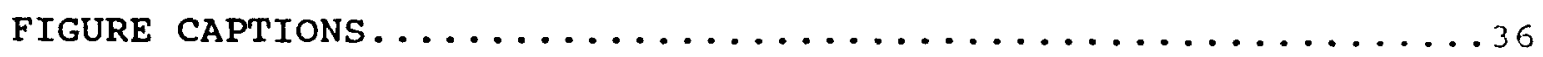

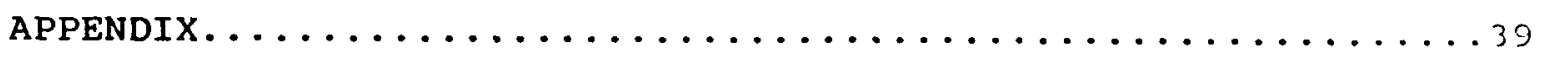

FIGURES $\ldots \ldots \ldots \ldots \ldots \ldots \ldots \ldots \ldots \ldots \ldots \ldots \ldots \ldots \ldots \ldots \ldots$

APPENDIX B

A DISTORTED WAVE IMPULSE APPROACH FROM ATOM-DIATOM

COLLISIONS.........................65

FIGURE CAPTIONS. ........................

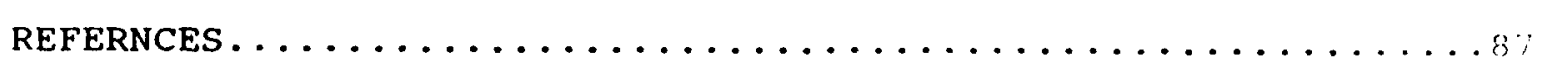

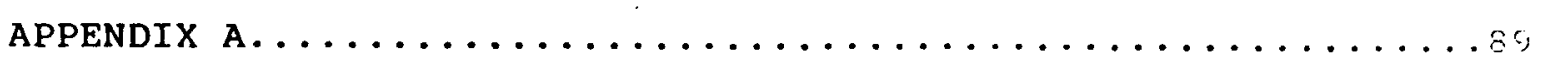

FIGURES $\ldots \ldots \ldots \ldots \ldots \ldots \ldots \ldots \ldots \ldots \ldots \ldots \ldots \ldots \ldots \ldots \ldots \ldots \ldots \ldots$

TABLE $1 \ldots \ldots \ldots \ldots \ldots \ldots \ldots \ldots \ldots \ldots \ldots \ldots \ldots \ldots \ldots \ldots \ldots \ldots \ldots$

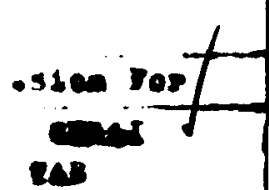

iomeec

i i i

Juib 1 esent len

DTIC QUALTYY INBPECTED 3

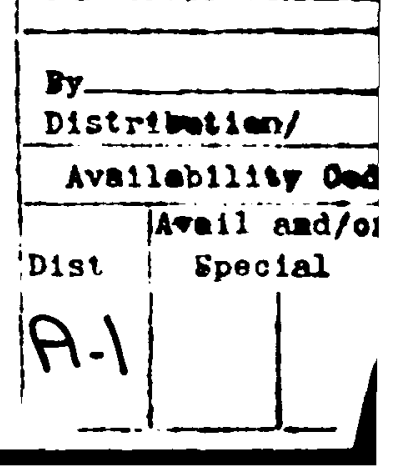




\subsection{INTRODUCTION}

During the first year, we have successfully applied the Impulse Approach (IA) to describe the Ar-CSF scattering system and have recently presented a comprehensive analysis of our findings ${ }^{l}$ reproduced in Appendix A. A major portion of our work has focused on the so-called ballistic effect, in which almost all of the relative translational energy of the atom-diatom system is converted into vibrational/rotational energy of the diatom. Several major findings have been reported. One major finding is that the ballistic effect found in the Ar-CsF system arises from two distinct kinematic mechanisms. The first mechanism giving rise to the ballistic effect is dominant when the laboratory scattering angle is very close to the laboratory angle of the centroid velocity. This mechanism involves the transfer of almost all of the relative translational energy into internal energy of the diatom. The second mechanism producing the ballistic effect is the rainbow singularity that arises when the recoil velocity of the alkali halide in the center of mass frame is perpendicular to its recoil velocity in the laboratory frame. Both mechanisms result from the kinematics, not the dynamics, of the collision process. We thus conclude that the ballistic effect should be observable for any two collision partners.

Further comparison of computed differential cross sections using the present IA method with experimental measured data had provided reasonable agreement in some cases and discrepancies in others. The present method limits the atom-diatom phenomenon to a sum of two atom-atom interactions. These atom-atom interactions have been, in all of the calculations for vibrationalrotational excitation of the diatoms approximated by hard core potentials. To arrive at an understanding of the influence of hard core potentials on the calculated differential cross sections, we carried out the IA calculations using exponential repulsive atom-atom interactions as well. We found that for the $\mathrm{Li}^{+}-\mathrm{N}_{2}$ scattering system, the resulting distributions of 
rotational probabilities are remarkably similar to those obtained with the hard core potentials. While the sum of the two atom-atom interactions appeared to be an adequate description of the atom-diatom potential at small distances, this model failed to describe its long range attractive part. To remedy this, we proposed to add to the sum of pair-interactions a centrally symmetric attractive potential located at the center of mass (c.m.) of the diatom, that "distorts" the incoming and outgoing waves in the scattering process. This Distorted Wave Impulse Approach (DWIA) is reported in a paper ${ }^{2}$ reproduced in Appendix $B$. An improved agreement between the DWIA calculated distributions of rotational transition probabilities and the experimental data suggest that the long range attractive part of the potential must be included in a model for small angle, high energy, atomdiatom collisions involving small amounts of energy transfer.

\subsection{DOUBLE COLLISION T MATRIX}

Research has continued into the further development of the existing atom-diatom impulse scattering theory. One basic premise of the impulse theory is that, during the collision process, the incident atom collides only with one end of the diatom, the other remaining a spectator. The operator which describes the energy transfer process that occurs during the collision of the incident atom with the diatom is known as the three-body $T$ matrix. The impulse theory reduces the three-body $T$ matrix to include only those terms representing collisions with only one end of the diatom. The result, called the single collision $T$ matrix, has been used in all of our calculations to date. The single collision $\mathrm{T}$ matrix ignores the possibility of collisions in which the incident atom collides with one end of the diatom, and then collides with the other end. We believe that this reduction may be responsible for the theory's inability to predict the ballistic effect for the Ar-CsI system, as well as its inability to reproduce the correct final rotational 
distributions of $\mathrm{N}_{2}$ after colliding with $\mathrm{Li}^{+}$at a relative translational energy of $4-7 \mathrm{eV}$. The two ends of CsI and of $\mathrm{N}_{2}$ are similar in mass, making the possibility of multiple collisions seem greater.

We have begun calculations employing the double-collision terms in the multiple-collision expansion of the three-body $T$ matrix. The inclusion of double-collision terms in a calculation using the IA applied to atom-diatom scattering is unprecedented. All previous calculations using the impulse method have neglected all but single-collision terms in the multiplecollision expansion, or Watson expansion, of the three-body $T$ matrix. We have suggested ${ }^{3}$ that some of the shortcomings of the Impulse Approach may be linked to this deficiency. For instance, the Impulse Approach has not been able to explain the ballistic effect for the Ar-CsI system studied by Herschbach. 4 Additionally, impulse calculations for the $\mathrm{Li}^{+}-\mathrm{N}_{2}$ system have yielded cross sections favoring too high a rotational excitation. 5 It is our belief that the inclusion of higher order terms in the Watson expansion will ameliorate some of these discrepancies.

We have developed several methods to evaluate the doublecollision terms, only one of which appears to be computationally feasible. The viable method is presently being applied to the $\mathrm{Li}^{+}-\mathrm{N}_{2}$ system. These formulations use the "part-classical" approximation to the impulse formalism, greatly reducing the amount of computation required while sacrificing an acceptable amount of accuracy.

\subsection{DWIA}

Applications of the IA model have produced results in reasonable agreement with experimental data, notably in studies of the relaxation of highly excited $\mathrm{KBr}$ by $\mathrm{Ar},{ }^{6}$ and in investigations of the "ballistic" effects for the CsF-Ar system. ${ }^{3}$ Meanwhile, IA calculations have not been able to reproduce the ob- 
served distributions of rotational transition probabilities of $\mathrm{N}_{2}$ in excitations caused by collisions with $\mathrm{Li}^{+} .5$ The computed distributions are too narrow and peak at values of the final rotational level $j$ ' that are too large. Interestingly, distributions obtained from classical trajectory calculations are also narrow but peak at values of $j$ ' that are too small. 5

To improve the computed distribution of rotational transition probabilities, we seek to employ more realistic potentials. We recall that in the IA, the total atom-diatom interaction potential is described by a sum of two atom-atom interactions. These atom-atom interactions are, in all of the IA calculations on vibro-rotational transitions previously published, approximated by hard core potentials, i.e., $V(r)=\infty$ for $r \leq d$ and $V(r)=0$ for $r>d$, $d$ being the suitably chosen hard core radius. A more realistic potential may be obtained by using atom-atom interactions expressed by exponential repulsive functions, instead of hard core functions. We have applied the IA model, using exponential repulsive atom-atom interactions, to compute differential cross sections for the rotationally inelastic processes $\mathrm{Li}^{+}$ $+\mathrm{N}_{2}(\mathrm{v}=0, j=2) \rightarrow \mathrm{Li}^{+}+\mathrm{N}_{2}\left(\mathrm{v}^{\prime}=0, j^{\prime}\right)$, as a function of $\mathrm{j}^{\prime}$, at a relative kinetic energy of $4.23 \mathrm{eV}$ and $\mathrm{cm}$. scattering angles of $49.2^{\circ}$ and $37.1^{\circ}$. The resulting distributions of rotational transition probabilities are remarkably similar to the ones obtained using hard core atom-atom interactions, being again narrow and peaking at values of $j$ ' that are too large when compared to the observed distributions. 6 These results suggest that IA results are insensitive to the shape (steepness) of the repulsive portion of the atom-atom potential.

While the simple sum of pair-interactions (either hard core or exponential repulsive) appears to be an adequate description of the total interaction potential at small distances between atom and diatom, it yields a poor representation of the long range attractive part. A much more realistic potential is obtained by adding to the sum of atom-atom interactions a central- 
ly symmetic potential, located at the center of mass (c.m.) of the diatom. In the newly derived Distorted Wave Impulse Approach (DWIA), 2 the added central potential "distorts" the incoming and outgoing waves in the collision process. The DWIA thus incorporates effects of the long range attractive part of the interaction potential into the calculation of atom-diatom collision cross sections using the Impulse Approach. The expression for the transition matrix $T$ in the DWIA formalism describes an overall collision process where the incoming particle is first scattered by the distorting potential located at the c.m. of the diatom, then scattered of one of its atoms, and, to maintain microscopic reversibility, finally scattered again by the distorting portential. The DWIA formalism was used to calculate differential cross sections for the rotationally inelastic processes $\mathrm{Li}^{+}+\mathrm{N}_{2}\left(\mathrm{v}=0, \mathrm{j}^{\prime} 2\right) \rightarrow \mathrm{Li}^{+}+\mathrm{N}_{2}\left(\mathrm{~V}^{\prime}=0, \mathrm{j}^{\prime}\right)$, as a function of $j '$, at a relative kinetic energy of $4.23 \mathrm{ev}$ and c.m.scattering angles of $49.2^{\circ}$ and $37.1^{\circ}$. It was found that the resulting distribution of rotational transition probabilities are in much better agreement with the experimental observations. 6 The DWIA distributions are broader and peak at values of $j$ ' that are smaller, reflecting the smaller momentum transfer found in the experiments. The more realistic potential, with its centrally symmetric component representing the long range attractive part, has produced a better agreement of the calculated differential cross sections with the measured ones.

The results of the investigations just described will be submitted shortly for publication in the Journal of Chemical Physics. 2

The derivation of the DWIA collision amplitude required a formulation of the IA collision amplitude in terms of the incoming and outgoing momenta $p_{3}$ and $p^{\prime}{ }_{3}$. This formulation is also crucial in the derivation of expressions for amplitudes corresponding to the higher collision terms in the multiple collision series expansion of the ' $\mathrm{T}$ matrix, 5 


$$
T=T^{(1)}+T^{(2)}+T^{(1)} G_{3} T^{(2)}+T^{(2)} G_{3} T^{(1)}+\ldots
$$

The Impulse Approach (IA) retains only the lowest order (i.e., single collision) terms. We have derived an expression for the transition amplitude corresponding to the double collision term $T^{(1)} G_{3} T(2)$. Computations are currently under way to evaluate the contributions of these double collision terms to the differential cross sections for the $\mathrm{Li}^{+}-\mathrm{N}_{2}$ system. These efforts represent the first attempts to go beyond the single collision terms in IA calculations, and to investigate the convergence of the multiple collision series.

\subsection{FUTURE WORK}

We intend to further the development of the three body $T$ matrix and DWIA methods and develop feasible techniques to evaluate the double-collision terms. Subsequent comparison of predicted results with experimental data should serve as a basis upon which the success of the technique may be judged.

\section{REFERENCES}

1. R.D. Sharma and J.M. Sindoni, "Inelastic and Ballistic Processes Resulting from SCF-Ar Collisions", I. Chem Phys (to be published).

2. H.Dothe and R.D. Sharma, "A Distorted Wave Impulse Approach for Atom-Diatom Collisions", J. Chem. Phys. (to be published).

3. J.M. Sindoni and R.D. Sharma, "Mechanism of Ballistic Collisions", Phys. Rev. A 45: R2659 (1992).

4. H.J. Leosch and D.R. Herschbach, "Ballistic Mechanisms for Vibrational and Roatational Energy Transfer in Ar + CsI Collisions", J._Chem. Phys. 57:2038. 
5. R.D. Sharma, P.M. Bakshii and J.M. Sindoni, "Impulse Formalism for Atom-Diatom Collisions", Phys Rev. A 43:189, (1991).

6. R.D. Sharma and J.M. Sindoni, Phys Rev. A, "Relaxation of Highly Vibrationally Excited KBr by Ar", 45:531 (1992).

7. R. Bittner, U. Ross and J. Peter Toennies, "Measurements of rotational and vibrational quantum transition probablities in the scattering of $\mathrm{Li}^{+}$from $\mathrm{N}_{2}$ and $\mathrm{CO}$ at center of mass energies of 4.23 and 7.07 eV", Chem. Phys., 65:733 (1976). 


\section{INELASTIC AND BALLISTIC PROCESSES RESULTING FROM CSF-Ar COLLISIONS *}

Ramesh D. Sharma, Phillips Laboratory, Optical Environment Division (GPOS), Hanscom Air Force Base, Massachusetts 01731.50)00.

Joseph M. Sindoni, Yap Analytics Inc., Lexington, Massachusetts 02173

\section{ABSTRACT}

This paper continues the study of inelastic and ballistic collisions for the CsF Ar system using the impulse approximation (IA). The IA expresses the atom-diatom potential as the sum of the two atom-atom potentials. The atom-atom interaction is approximated by a hard core potential and the laboratory differential cross sections are calculated for an initial relative translational encrgy of $1.0 \mathrm{eV}$ as a function of the laboratory recoil velocity of CsF. The calculated differential cross sections are in excellent agreement with the experimental measurements for all eight laboratory scattering angles for which the data are available. While the calculated results show no significant dependence on the initial relative velocity or on the initial vibrational quantum number of $\mathrm{CsF}$, they do show a systematic variation with the initial rotational quantum number--the ballistic effect is more pronounced than that observed experimentally for initial quantum rotational numbers less than 30 and is not pronounced enough for rotational quantum numbers more than 100. Two mechanisms give rise to the ballistic peak. The first one is dominant when the laboratory scattering angle is equal, or nearly equal, to the laboratory angle of the centroid velocity. This mechanism transfers almost all of the relative translational energy into the internal energy of the diatom and magnifies the center-of-mass (c.m.) differential cross section almost a million times. This is due to a singularity in the Jacobian at very small c.m. recoil $\because$ Joumal Cnem. Phos. (" ") be publishoul) 
velocities, which physically means that a small solid angle in the laboratory frame can collect the signal from all $4 \pi$ steradians in the c.m. frame. The second mechanism producing the ballistic peak, also determining the smallest and the largest laboratory scattering angles, is the rainbow-like singularity called edge effect. This mechanism becomes operative when the recoil velocity of the alkali halide in the c.m. frame is perpendicular to its recoil velocity in the laboratory frame. While the dynamics of the collision leads to a conversion of the proper amount of relative translational energy into internal energy of the diatom, the kinematic singularities mentioned above magnify the relevant c.m. differential cross sections leading to the observed ballistic effect. The ballistic effect, therefore, should be observable for any two collision partners under appropriate circumstances. The simple alom-diatom potential reproduces the experimental results very well, because: (i) for inelastic scattering, the experimental observations correspond to large center of mass scattering angles for which the attractive part of the potential makes little contribution to the scattering process, (ii) for ballistic scattering, only the repulsive portion of the potential can cause a large amount of energy exchange between the relative translational and the internal degrees of freedom and, (iii) the calculated cross sections are insensitive to the details of the repulsive portion of the potential. A number of consequences of the theory, including the conclusion that the alkali halide beam in the experiments is rotationally unrelaxed, are discussed.

\section{INTRODUCTION}

In a series of experiments, Herschbach and co-workers [1-3] have measured the differential cross sections for the scattering of $\operatorname{CsX}(X=F, I)$ by $\operatorname{Ar}$ as a function of the laboratory recoil velocity of $\mathrm{CsX}$ by crossing the two beams, at a right angle to each other, at a relative translational energy of about $1.0 \mathrm{eV}$. In addition to a peak observed around the elastically scattered CsX (pscudoclastic peak, formerly called the elastic peak [4]), another peak, almost as strong as the pscudoelastic peak and named the ballistic 
peak, was observed in the vicinity of the recoil velocity corresponding to the motion of the center of mass. Obviously, those molecules that constitute the ballistic peak have a substantial fraction of their relative translational energy converted into internal energy during the collisions. A theory of the ballistic effect is thus a theory of collisions during which a large fraction of the relative translational energy is converted into internal energy. The pseudoelastic peak observed in the experiments $[1-3]$ corresponds to large angle scattering in the center of mass (c.m.) frame. The theory of the collisions comprising the pseudoelastic peak, also under consideration here, is therefore a theory of large angle elastic and inelastic scattering.

A model for the inelastic and ballistic transitions for the CsF-Ar system constructed earlier [4] produces excellent agreement with the experimentally measured differential cross section as a function of the laboratory recoil velocity of $\mathrm{CsF}$ at the laboratory scattering angles of $30^{\circ}$ and $60^{\circ}$. According to this model, the inelastic (ballistic) collisions result when Ar strikes the Cs (F) end of CsF. The inelastic collisions involve modest change in the rotational quantum number $(\Delta j=50)$; the ballistic collisions, on the other hand, to conserve angular momentum and energy, must involve large changes in the rotational quantum number $(\Delta \mathrm{j}$ up to about 200$)$. Our model for the ballistic and large angle inelastic collisions has been able to give a quantitative explanation of the experimental observations of the CsF-Ar system, but it has not been able to explain the ballistic effect observed in the CsI-Ar system. Our model, however, has pointed out that the ballistic effect should not be observed in the $I_{2}$ - Ar system at about $1 \mathrm{eV}$ relative translational energy because the $\mathrm{I}_{2}$ beam is rotationally cold (rotational temperature $\sim 250^{\circ}$ for the $\mathrm{I}_{2}$ beam vs. $\sim 1000^{\circ}$ for the CsI beam), a prediction in agreement with the experimental results. The present theory has, in addition, predicted a ballistic peak for the $I_{2}$-Ar system at relative translational energy of about $0.12 \mathrm{eV}$ [4]. This prediction of our model is in contrast to the prediction of an earlier model [5] of atom-diatom collisions, which concludes that only about $25 \%$ of the initial relative 
translational energy should be converted into internal energy during an $\mathrm{Ar}-\mathrm{I}_{2}$ encounter. independent of the initial internal energy of the diatom or the atom-diatom relative translational energy. The same model [5] predicts a transfer of about $96 \%$ of the relative translational energy into internal degrees of freedom during an Ar-FCs encounter, again independent of the initial internal energy of the diatom or the atom-diatom relative translational energy. It was pointed out earlier [4] that when the laboratory scattering angle is the same, or nearly the same, as the laboratory angle of the c.m. velocity, a transfer of more than $96 \%$ of the relative translational energy into internal degrees of freedom is required to observe a ballistic effect. It will be shown later, in this article, that when the laboratory scattering angle is much larger than, or much smaller than, the laboratory angle of the c.m. velocity, a transfer of a mere $75 \%$ of the relative translational energy into internal degrees of freedom may lead to a ballistic effect. Again this is in conflict with the earlier model [5] of impulsive collisions. Our model, since it has not explained all the pertinent experimental observations, is only the first step in fully understanding the mystery of ballistic collisions. Nevertheless, by virtue of the excellent agreement between the calculated and the measured results for the CsF-Ar system, the predictions it has made, and the experiments it has suggested, our model represents a valuable first step. For this reason it is considered appropriate to present a more complete theory for the CsF-Ar system, expanding on the previous results as well as presenting new ones.

Before presenting the detailed theory of the ballistic effect, it is necessary to discuss the transformation of the differential cross sections from the c.m. frame to the laboratory coordinates. This transformation is an important link bridging the results calculated in the c.m. frame to the experimental results measured, of course, in the laboratory frame. Section 2 discusses this transformation, which is derived in the Appendix. Section 3 gives a brief account of the impulse approach (IA) for the atomdiatom collisions with a special emphasis on the part-classical ( previously called "semi- 
classical") formalism. The calculation is still fully quantum, but by treating the two-body dynamics classically it is possible to save a great deal of computing time without appreciable loss of accuracy. Section 4 briefly describes the computational procedures used. Section 5 discusses the two mechanisms leading to the ballistic peak. The results of the calculation are compared with the experimental measurements in Section 6. Our state-resolved three dimensional fully quantum calculation is examined in greater detail in Section 7 and some of its more interesting consequences are pointed out. Concluding remarks and lessons learned comprise the last section, Section 8.

\section{TRANSFORMATION OF THE DIFFERENTIAL CROSS SECTION FROM THE CENTER OF MASS TO THE LABORATORY SYSTEM}

The transformation between the $c . m$. and the laboratory coordinate systems has been the subject of several studies [6-8]. We give a particularly simple derivation of the Jacobian for inelastic scattering. We then use this derivation as the starting point for the discussion of singularities and connect it with the previous work on the ballistic collisions.

The differential cross section in the laboratory coordinate system $\sigma_{L}\left(\theta_{L}, \phi_{L}\right)$ is related to the differential cross section in the center of mass coordinate system $\sigma(\theta, \phi)$ by the relation

$$
\sigma_{\mathrm{L}}\left(\theta_{\mathrm{L}}, \phi_{\mathrm{L}}\right)=\lim _{\Delta \Omega_{\mathrm{L}}, 0} \frac{\int_{\Omega_{m}}^{s_{\mathrm{S}}} \sigma(\theta, \phi) \mathrm{d} \Omega}{\Delta \Omega_{\mathrm{L}}},
$$

where $\theta, \phi$ are the polar and the azimuthal angles and $\Omega$ is the solid angle in the c.m. coordinate system; the subscript $L$ indicates that the angles are measured in the laborittory cuordinate system. $\Omega_{\mathrm{M}(\mathrm{m})}$ denotes the maximum (minimum) c.m. solid angle corresponding to laboratory solid angles $\Omega_{\mathrm{l}} \pm \Delta \Omega_{\mathrm{l}}$. Defining 


$$
\left(\frac{v_{2}^{\prime}}{\omega_{2}^{\prime}}\right)^{2} \equiv \gamma_{1}^{2}
$$

and taking the limit as $\Delta \Omega_{\mathrm{L}}$ approaches zero, the expression for the Jacobian is writur: as

$$
\sigma_{L}\left(\theta_{1}, \phi_{L}\right)=\left|\frac{d \Omega_{1}\left(\theta_{1}, \phi_{1}\right)}{d \Omega(\theta, \phi)}\right|^{-1} \sigma(\theta, \phi)
$$

where $\theta, \phi$ and $\theta_{\mathrm{L}}, \phi_{\mathrm{L}}$ are polar and azimuthal scattering angles in the c.m. frame and the laboratory frame, respectively. Defining $\gamma \equiv\left(\frac{v_{c, n}}{\omega_{2}^{\prime}}\right)$ as the ratio of the velocity of the centroid in the laboratory frame to the recoil velocity of the observed particle in the c.m. frame, we show, in the appendix ,

$$
\begin{aligned}
\left|\frac{d \Omega_{L}\left(\theta_{L}, \phi_{L}\right)}{d \Omega(\theta, \phi)}\right|^{\prime} & =\frac{2 \gamma_{1}^{3}}{\left|1+\gamma_{1}^{2}-\gamma^{2}\right|} \\
& =\frac{\gamma_{1}^{2}}{\left|\left(\hat{\omega}_{2}^{\prime} \cdot \hat{v}_{2}^{\prime}\right)\right|}
\end{aligned}
$$

where $\hat{v}_{2}^{\prime} \cdot \hat{\omega}_{2}^{\prime}$ is the cosine of the angle between the recoil velocities of the species detected in the laboratory and the c.m. frames. It is shown in the appendix that equation (4) is identical to the one given by Schiff [9] when one of the particles is initially stationary. This expression becomes infinite when $\omega_{2}^{\prime}$ approaches zero, i.e., when the c.m. recoil velocity becomes very small, and also when the $\mathrm{c} . \mathrm{m}$. and the laboratory recoil velocities are perpendicular.

When $\omega_{2}^{\prime}$ approaches zero, the direction of $v_{2}^{\prime}$, the laboratory recoil velocity. coincides with that of the centroid velocity, $v_{c . m}$. It is pointed out in the appendix that in 
this case the Jacobian becomes a constant and is simply equal to $\frac{4 \pi}{\Delta \Omega_{L}}, \Delta \Omega_{L}$ being the laboratory solid angle seen by the detector. For a $0.25^{\circ}$ wide detector, the Jacobian is equal to $8.4 \times 10^{5}$. Herein lies part of the mystery of the ballistic effect! A million fold enhancement of the cross sections for the processes that lead to the conversion of almost all of the relative translational energy into internal energy causes very small cross sections in the c.m. frame to stand out in the laboratory frame. This hypothesis is the basis of the proposal for the production of the state-selected and velocity-selected molecular beams [4].

When the laboratory and c.m. recoil velocities are perpendicular, the Jacobian given above becomes infinite. It is shown in the appendix that for a given final vibrational-rotational state, i.e., for constant magnitude of $\omega_{2}^{\prime}$, the laboratory scattering angle as a function of c.m. scattering angle goes through a maximum (minimum) when the laboratory scattering angle is greater (simaller) than the centroid velocity angle $\theta_{2}$. An increase (decrease) in the c.m. scattering angle leads to a decrease (increase) in the laboratory scattering angle. The situation encountered here is the same as that encountered in the study of rainbows [10] where the extremum in the deflection function as a function of impact parameter leads to infinite classically-calculated differential cross section. These two rainbows-like singularities, called the edge effect [11], correspond to a lower and an upper bound for the laboratory scattering angle for a given final vibrational-rotational state. The rainbow-like singularities encountered here are due to kinematic effects and are distinct from the rainbows due to the dynamical effects discussed previously [12].

In the appendix the Jacobian for the edge effect is shown to be equal to 


$$
\begin{aligned}
\left|\begin{array}{c}
d \Omega_{1} \\
d \Omega
\end{array}\right|^{\prime} & =\left|\begin{array}{c}
\sin \theta_{1} \Delta \theta_{1} d \phi_{1} \\
\left.\sin \left(\theta+\theta_{1}-\theta_{2}\right) \Delta \theta d \phi\right|^{\prime}
\end{array}\right|^{\prime} \\
& \equiv\left|\frac{8 \gamma_{1}^{3}}{\Delta \theta_{L}}\right|^{2}
\end{aligned}
$$

where the angle $\Delta \theta_{\mathrm{L}}$ is the width of the polar angle of the detector. The Jacobian given by equation (5) for $\gamma_{1} \approx 5$ and $\Delta \theta_{\mathrm{L}}=0.250$ degrees can have a value roughly between 100) and 1000 depending upon the laboratory scattering angle, i.e., it can have a value between 4 and 40 times that given by $\gamma_{1}^{2}$ alone. This effect is not as dramatic as that due to resonant transfer of energy from relative translational motion into rotational and vibrational motion of the diatom. However, in this case the combined contributions from different transitions can lead to an equally strong ballistic peak.

\section{ATOM-DIATOM IMPULSE FORMALISM}

The complete formulation of the impulse approach to atom-diatom collisions was given earlier [13]. Here we give a brief outline for completeness with an emphasis on the part-classical approximation to the exact equations. In this formulation the dynamics of two-body collisions is treated classically while the remainder of the calculation is carried out using quantum mechanics. It has been found that this procedure leads to substantially reduced computing time while the error introduced has never been found to exceed $10 \%$. In the present calculation more than one hundred thousand differential cross sections had to be computed; the use of this approximation has kept the computation time manageable.

The interaction potential of the atom-diatom system in the impulse formulation is the sum of the spherically symmetric atom-atom potentials, i.e.,

$$
V\left(y_{1}, y_{2}\right)=V_{1}\left(y_{1}\right)+V_{2}\left(y_{2}\right) \text {, }
$$

where $V_{i}$ and $y_{i}$ are, respectively, the interaction potential and the distance between the incident atom and atom $\mathrm{j}$ of the diatom. The distance between the incident atom 3 and atoms 1 and 2 of the diatom is written as 


$$
y_{0}=\left[r^{2}+2 \alpha_{0} r y_{3} \cos \gamma_{0}+\left(\alpha_{0} y_{3}\right)^{2}\right]^{\prime}
$$

where $s=1,2$ and $r$ is the distance between the atom and the center of mass (c.m.) of the diatom; $y_{3}$ is the internuclear distance of diatom $1-2$,

$$
\alpha_{s}=(-1)^{s} m_{s} /\left(m_{1}+m_{2}\right),
$$

$\mathrm{m}_{\mathrm{S}}$ is the mass of spectator atom, and $\gamma_{\mathrm{a}}$ is the angle between the internuclear axis $\mathrm{y}_{3}$ and the line $\mathbf{r}$ joining the atom to the c.m. of the diatom. Eigenfunctions $\psi_{v, j, m}$ of the Hamiltonian for the diatomic molecule

$$
\mathrm{H}_{\text {DIATOM }}=\frac{\mathrm{q}_{3}^{2}}{2 \mu_{12}}+\mathrm{V}_{3}\left(\mathrm{y}_{3}\right)
$$

are the wave functions for the vibrational and rotational motion of the diatom. The momenta are written in the Jacobi notation; $\mathbf{p}_{\mathrm{a}}$ is the momentum of particle a with respect to the c.m. of bc and $q_{a}$ is the relative momentum of particles bc. Similarly, $\mathbf{r}_{\mathrm{a}}$ is the distance of atom a from the c.m. of $b c, y_{a}$ is the distance between $b$ and $c$, and $\mathrm{V}_{3}$ is the intramolecular potential of the diatom 1-2. $\mu_{\mathrm{ij}}$ is the reduced mass of the atoms $i$ and $j$, while the reduced mass of $a$ and $b c$ is written as $\mu_{a}$.

The Watson expansion, a multiple-collision expansion of the three-body $T$ matrix, is written as [14]

$$
T=T^{(1)}+T^{(2)}+T^{(1)} G_{3} T^{(2)}+T^{(2)} G_{3} T^{(1)}+\ldots
$$

where $\mathrm{T}^{(\mathrm{i})}$ is the three-body transition matrix describing the collision of the incident atom with atom $\mathrm{j}$ of the diatom, $\mathrm{i}$ being the spectator atom. $\mathrm{G}_{3}$ is the propagator corresponding to the unperturbed Hamiltonian $\mathrm{H}_{0}$, viz,

$$
H_{0}=\frac{p_{3}^{2}}{2 \mu_{3}^{2}}+H_{\text {DIATMM }}
$$

and,

$$
G_{3}(z)=\left(z-H_{0}+i \eta\right)^{\prime}
$$


The first two terms on the right hand side of equation (10) are the single-collision terms. These terms are obtained by summing all the diagrams involving the collision of the incident atom with atom 1 or atom 2. The graphs representing the collision of the incident atom with atom 1 (2) followed by further collisions of the incident atom with atom 1 (2) are contained in these terms [14]. The next two terms are the doublecollision terms and are obtained by summing all the diagrams involving the collisions of the incident atom first with atom 1 (2) followed by collision with atom 2 (1) [14]. In the impulse calculation only the first two terms, i.e., only the single collision terms, are retained.

We can write the differential cross section for scattering from initial state $i$ to final state $f$ as

$$
\frac{d \sigma}{d \Omega}\left(i \equiv v j p_{3} \rightarrow f \equiv v^{\prime}, j^{\prime}, p_{3}^{\prime} ; q\right)=\frac{p_{3}^{\prime}}{p_{3}}(2 j+1)^{-1} \mu_{3}^{2}\left(\frac{2 \pi}{\hbar}\right)^{4}\left|T_{i f}\right|^{2}
$$

where the scattering angle $\theta$ and momentum transfer vector $q$ are related by

$$
q^{2}=\left(p_{3}^{\prime}\right)^{2}+\left(p_{3}\right)^{2}-2 p_{3} p_{3}^{\prime} \cos \theta
$$

and,

$$
\left|T_{i f}\right|^{2}=\sum_{m, m^{\prime}}\left|\left\langle\phi_{3}^{\prime}|T| \phi_{3}\right\rangle\right|^{2}
$$

$\phi_{3}$ and $\phi_{3}^{\prime}$ being the eigenfunctions of $H_{0}$ in the initial and final states, respectively, and,

$$
\mathrm{T}_{\text {if }}(\mathbf{q})=\left(\sum_{\mathbf{s}=1,2} \mathrm{~T}_{\mathrm{if}}^{(\mathbf{x})}(\mathbf{q})\right)
$$

The two terms on the right hand side of equation (16) are the single collision terms. When the incident atom collides with one of the atoms of the diatom the other atom, the one not participating in the collision process, is termed the spectator. For this reason 
the impulse calculation is also called the quantum mechanical spectator model. We recall that

$$
\left\langle\phi_{3}^{\prime}\left|T^{(s)}(q)\right| \phi_{3}\right\rangle=\int d y_{3} \phi_{3}^{\prime *}\left(y_{3}\right) \exp \left(-i \alpha_{s} q \cdot y_{3}\right) \Psi^{(s)}\left(y_{3}\right),
$$

where,

$$
\psi^{(s)}\left(\mathrm{y}_{3}\right) \equiv(2 \pi)^{-\frac{3}{2}} \int \mathrm{d} \mathrm{q}_{3} \exp \left(-\mathrm{iq}_{3} \cdot \mathrm{y}_{3}\right)\left\langle\left.\mathrm{q}_{\mathrm{s}}^{\prime}\right|^{(\mathrm{s})} \mid \mathrm{q}_{\mathrm{s}}\right\rangle \phi\left(\mathrm{q}_{3}\right)
$$

is the wave function of the diatom modified by the two-body scattering process. To develop a feeling for the nature of $\psi^{(0)}\left(y_{3}\right)$, it is useful to look at the two-body t-matrix as an operator in the momentum space which modifies the diatom wave function $\phi\left(\mathbf{q}_{3}\right)$. Equation (18) transforms the modified wave function back to the coordinate space. If one recalls that the center of mass acquires a momentum equal to $\alpha_{s} q$ during the collision when $\mathrm{s}$ is the spectator atom, equation (17) may be looked upon as the overlap integral of the final-state wave function with the initial-state wave function that is modified by the collision. This view also connects the impulse approach discussed here with the theory of transitions due to sudden perturbations, e.g., atomic transitions accompanying beta ray emission[15].

It was mentioned earlier that we will evaluate the two-body t-matrix in equation (18) using classical mechanics. In classical mechanics, a collision between two hard spheres leads to the reversal of the component of the momentum normal to the surfaces of the two spheres at the point of contact, while the other two components of the momentum remain unchanged [16]. In other words, if a momentum change $q$ occurs during the collision, the initial momentum $-q / 2$ becomes the final momentum $+q / 2$, while the components of momentum perpendicular to $q$ remain unchanged. Further, the transverse components of the momentum, which in classical mechanics do not enter the equations of motion, may have any value. It was shown earlier [13] that this result also holds for scattering using the impulse approach, provided the scattering angle is larger 
than about $15^{\circ}$. This amounts to evaluating equation (18) by setting the component of $\mathbf{q}_{3}$ along $\mathbf{q}$ equal to

$$
\mathbf{q}_{3} \cdot \hat{\mathbf{q}}=-\left(\alpha_{\mathbf{g}} \mathrm{q} / 2\right)+\mu_{12} \Delta \varepsilon /(\alpha, q)
$$

where $\hat{\mathbf{q}}$ is a unit vector along the direction of the transferred momentum and $\Delta \varepsilon$ is the difference in internal energy of the diatom between the final and the initial states. The components of $\mathbf{q}_{3}$ perpendicular to $\mathbf{q}$ are set equal to zero. It was also pointed out carlier [13] that to evaluate the two-body t-matrix for a fixed value of $q_{3}$, the momentum due to the vibrational and rotational motion of the diatom, is to approximate it by its spherically symmetric component, i.e.,

$$
\left\langle q_{.}^{k}\left|t^{(s)}\right| q_{1}\right\rangle \approx t_{00}\left(q_{3}, p_{3}, q\right) Y_{00}\left(\hat{q}_{3}\right)=(4 \pi)^{-\frac{1}{2}} t_{00}\left(q_{3}, p_{3}, q\right)
$$

Equation (20) has the same structure as the peaking approximation, which evaluates the two-body t-matrix setting $q_{3}=-\alpha_{s} q / 2$, i.e., using only the first term on the right hand side of equation (19). Use of equation (19), however, gives results which, unlike the results obtained by using the peaking approximation [17], are time reversal invariant[13]. This approach is called the part-classical approximation, previously called semi-classical (new name is given to avoid confusion with other usage of the term semi-classical in scattering theory [10]), because it has one foot in classical mechanics. Equation (20) has been extensively used by us for calculating differential cross sections and has provided answers always within $10 \%$ of those given by a spot-check of the exact calculation $[4,18]$.

Recalling that $\phi\left(q_{3}\right)$ is the wave function of the initial state in the momentum representation, i.e.,

$$
\phi\left(\mathbf{q}_{3}\right)=\left(\frac{2}{\pi}\right)^{\frac{1}{2}} \mathrm{i}^{\mathrm{j}} \mathrm{I}_{\mathrm{vj}}\left(\mathrm{q}_{3}\right) \mathrm{Y}_{\mathrm{jm}}\left(\hat{\mathbf{q}}_{3}\right)
$$

where $v, j, m$ are the initial vibrational, rotational, and magnetic quantum numbers. respectively, and, 


$$
I_{v, j}\left(q_{3}\right)=\int_{0}^{\infty} d y_{3} y_{3}^{2} \chi_{v, j}\left(y_{3}\right) j_{j}\left(q_{3} y_{3}\right)
$$

where $\chi_{v j}$ is the vibration-rotation wave function in the coordinate space, we can integrate equation (18) over $q_{3}$. Using the Raleigh expansion of the plane wave, i.e.,

$$
\exp \left(-i q_{3} \cdot y_{3}\right)=4 \pi \sum_{1, m}(-i)^{l} Y_{l m}\left(\hat{\mathbf{y}}_{3}\right) Y_{l m}^{*}\left(\hat{q}_{3}\right) j_{1}\left(q_{3} y_{3}\right)
$$

we obtain,

$$
\psi^{(s)}\left(\mathbf{y}_{3}, \mathbf{q}, \mathbf{p}_{3}\right)=(4 \pi)^{-\frac{1}{2}} K_{\mathrm{vj}}^{(s)}\left(\mathrm{y}_{3}, \mathbf{q}, \mathbf{p}_{3}\right) \mathrm{Y}_{\mathrm{jm}}\left(\hat{\mathbf{y}}_{3}\right)
$$

where,

$$
K_{v j}^{(0)}\left(y_{3}, p_{3}, q\right)=\frac{2}{\pi} \int_{0}^{\infty} d q_{3} q_{3}^{2} j_{j}\left(q_{3} y_{3}\right) t_{00}^{(0)}\left(q_{3}, p_{3}, q\right) !_{v, j}\left(q_{3}\right)
$$

A comparison of equations (24) and (25) with equations (21) and (22) shows the similarity of their structure and provides further basis for the statement that $\psi^{(0)}$ is the modified wave function for the internal motion of the diatom. Substituting equations (18) through (25) into equation (17), we get

$$
\left\langle\phi_{3}^{\prime}\left|T^{(s)}(q)\right| \phi_{3}\right\rangle=\left(\frac{1}{4 \pi}\right)^{\frac{1}{2}} \int d y_{3} \phi_{3}^{\prime *}\left(y_{3}\right) Y_{j m}\left(\hat{y}_{3}\right) \exp \left(-i \alpha_{s} q \cdot y_{3}\right) K_{v j}^{(s)}\left(q, p_{3}, y_{3}\right)
$$

Again using the Raleigh expansion of the plane wave and writing $\phi_{3}^{\prime}\left(y_{3}\right)=\chi_{v^{\prime}, j}\left(y_{3}\right) Y_{j m^{\prime}}\left(\hat{y}_{3}\right)$, where primes denote the vibrational and rotational levels $v^{\prime}, j^{\prime}$ of the final state, we get

$$
\left\langle\phi_{3}^{\prime}\left|T^{(0)}(q)\right| \phi_{3}\right\rangle=\sum_{l}( \pm i)^{\ell}\left(\frac{|\ell \| j| j \mid}{\left[j^{\prime} \mid\right.}\right)^{\frac{1}{2}} N_{v \ell^{\prime \prime j^{\prime}}}^{(0)}\left(\mathbf{q}, p_{3}\right) C\left(j / j^{\prime} ; m, m^{\prime}-m\right) C\left(j \ell j^{\prime} ; 00\right) Y_{\ell, m^{\prime} \cdot m}^{*}(\hat{q}),
$$


where C's are the Clebsch-Gordan coefficients, $[j] \equiv(2 j+1)$ and,

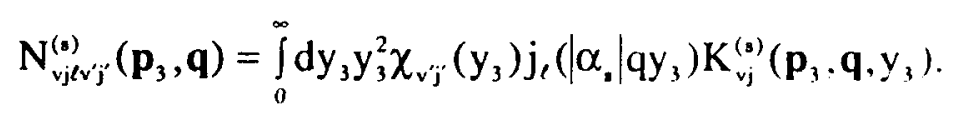

Taking our space fixed $z$-axis along $\mathbf{q}$, and using the relation

$$
Y_{\ell . m}^{*}(0,0)=\left(\frac{[\ell]}{4 \pi}\right)^{\frac{1}{2}} \delta_{m .0}
$$

we get,

$\left\langle\phi_{3}^{\prime}\left|T^{(\mathbf{s})}(\mathbf{q})\right| \phi_{3}\right\rangle=(4 \pi)^{-\frac{1}{2}} \sum_{\ell}( \pm \mathrm{i})^{\ell}\left(\frac{[\mathrm{j}]}{\left[\mathrm{j}^{\prime}\right]}\right)^{\frac{1}{2}}[\ell] \mathrm{N}_{\mathrm{vj}^{\prime} / v^{\prime \prime} \mathrm{j}^{\prime}}\left(\mathbf{q}, \mathbf{p}_{3}\right) \mathrm{C}\left(\mathrm{j} \ell \mathrm{j}^{\prime} ; \mathrm{m}, 0\right) \mathrm{C}\left(\mathrm{j} \ell \mathrm{j}^{\prime} ; 00\right)$.

Using the relation [19]

$$
\sum_{m} C\left(j \ell j^{\prime} ; m 0\right) C\left(j \ell^{\prime} j^{\prime} ; m 0\right)=\left(\frac{\left[j^{\prime}\right]}{[\ell]}\right)^{\frac{1}{2}} \delta_{\ell, \ell^{\prime}}
$$

we can write, using equations (13) and (16),

$$
\begin{aligned}
\frac{d \sigma}{d \Omega}\left(i \equiv v_{3} p_{3} \rightarrow f \equiv v^{\prime}, j^{\prime}, p_{3}^{\prime} ; q\right)= & \frac{p_{3}^{\prime}}{p_{3}}(2 j+1)^{-1} \mu_{3}^{2}\left(\frac{2 \pi}{\hbar}\right)^{4} \\
& \times\left(\frac{1}{4 \pi}\right) \sum_{\ell=\left|j^{\prime}-j\right|}^{j^{\prime}+j}[\ell] C^{2}\left(j \ell j^{\prime} ; 00\right)\left|N_{v j \ell v^{\prime} j^{\prime}}^{(1)}+(-)^{\ell} N_{v j \ell v^{\prime} j^{\prime}}^{(2)}\right|^{2} .
\end{aligned}
$$

Equation (32) is our final result and is obtained by adding the amplitudes for scattering from the two scattering centers. 


\section{COMPUTATIONS}

In this section we briefly describe the computational procedures followed. These procedures are the same as in the previous work on the collision of alkali halide molecules with argon $[4,18]$. Briefly, since the ground state of $\mathrm{CsF}$ is ionic, the Ar-CsF potential is taken to be the sum of $\mathrm{Ar}-\mathrm{Cs}^{+}$and $\mathrm{Ar}^{-} \mathrm{F}^{-}$potentials. Only the repulsive portion of the Ar-ion potential, which is approximated by a hard core potential, is used in the present calculations. The hard core radii for the $\mathrm{Ar}-\mathrm{Cs}^{+}$and $\mathrm{Ar}^{-} \mathrm{F}^{-}$potentials are assumed to be given by the corresponding parameters for the Ar-Xe and Ar-Ne potentials [20]. The parameters for the diatom potential are taken from Huber and Herzberg [21]. The potential function thus obtained is extrapolated to larger internuclear distances using a Padé $[2,2]$ approximant. Wave functions for the internal motion of the diatom are obtained by solving the one dimensional Schrödinger equation, containing the centrifugal term for the rotational motion, using Numerov's method.

\section{MECHANISMS GIVING RISE TO THE BALLISTIC PEAK}

Before we compare the calculated differential cross sections with the experimental values, it is desirable that we discuss the physical basis for the ballistic peak. Figure 1, plotted using the points taken from the earlier work [2], gives a plot of the laboratory differential cross section as a function of $\mathrm{CsF}$ recoil velocity and illustrates the experimental data we are trying to model. The ballistic peak on the left, near the centroid velocity, represents the signal from those molecules that have small c.m. recoil velocities and which carry large amounts of internal excitation [1]. The ballistic peak was shown [4] to arise when Ar strikes the F end of CsF. The peak on the right, called the pseudoelastic peak [4], centered at the recoil velocity corresponding to elastic scattering, arises [4] when Ar strikes the Cs end of CsF and consists of inelastic 
transitions involving modest changes $\left(\frac{|\Delta E|}{E} \leq 0.35\right)$ of collision energy. An earlier model of impulsive atom-diatom collisions predicts [5] a transfer of only $14 \%$ of the initual relative translational energy into internal energy, i.e., $\left(\frac{|\Delta E|}{E}=0.14\right)$, when Ar hits the $C$ s end of $\mathrm{CsF}$. The results of our calculation and those of the earlier model are in disagreement. Comparison of our calculation with the experimentally observed pseudoelastic peak will decide if our theory is correct.

It was shown earlier [4] that when one is looking along or close to the direction of the centroid velocity, most of the contribution to the ballistic peak comes from the transitions which convert more than $97 \%$ of the relative translational energy into internal energy. Further, the signal from a transition which converts more than $99 \%$ of the relative translational energy into internal motion (resonant transition) may be larger than the signal from any other transition by a factor of about 2-3. It was also pointed out that the c.m. differential cross section for the resonant transitions is within a few percent of the neighboring non-resonant transitions. What sets the resonant transitions apart from the nearby transitions is the large Jacobian of transformation from the c.m. to the laboratory coordinate system, which is very sensitive to the fraction of relative translational energy converted into internal energy. The expression for the Jacobian is derived in the appendix. Figure 2 is a plot of the Jacobian for the transformation from the c.m. to the laboratory coordinates, for the laboratory scattering angle equal to the angle of the centroid velocity, as a function of the fraction of the relative translational energy converted into internal energy. It is readily seen from this figure why the differential cross section for the resonant transitions in the laboratory frame is so much larger than the neighboring nonresonant transitions.

When the recoil velocities of the alkali halide molecules in the c.m. frame and the laboratory frame are perpendicular, the laboratory scattering angle for two values, a 
maximum and a minimum, displays an extremum as a function of the c.m. scattering angle. The laboratory scattering cross section, for these two values of laboratory scattering angles, exhibits a rainbow-like singularity. Figure 3 gives a plot of the differential cross section, obtained by summing over the two branches in figure $4 \mathrm{~A}$, in the laboratory (c.m.) frame as a of function laboratory (c.m.) scattering angle. The collision parameters are given in figure $3 \mathrm{~A}$. The minimum (maximum) laboratory scattering angle of $30^{\circ}\left(75^{\circ}\right)$ correspond to c.m. scattering angles of $18^{\circ}\left(117^{\circ}\right)$. An order of magnitude enhancement of the differential cross section due to the kinematic rainbow-like singularity (edge effect) is seen. The values of the minimum and maximum laboratory angles are dependent upon the transition under consideration. These kinematic rainbows, called the edge effect [11], are the cause of the ballistic peak when the laboratory scattering angle is much different from the direction of the centroid velocity.

\section{COMPARISON OF THE CALCULATION WITH THE EXPERIMENTAL}

\section{RESULTS}

The calculated results are compared with the experimental results after they are scanned over by a normalized Gaussian function with the resolution ratio [1] $\mathrm{R} \equiv$ $\left(\Delta v_{1} / v_{1}\right)=\left(19.3+0.034 v_{1}\right)$, where $R$ is expressed in $\%, \Delta v_{1}$ is the full width at half maximum of the Gaussian, and $v_{1}$ is in units of $\mathrm{m} / \mathrm{s}$. To understand the role of the resolution ratio in the experimental results, we plot in figure 4 the output signal (dashed line) in arbitrary units as a function of the velocity of Ar when the input (solid line) is given by $P\left(v_{1}\right)=v_{1}{ }^{3} \exp \left(-\left[\left(v_{1}-<v_{1}>\right) / \delta v_{1}\right]^{2}\right)$ with $\left\langle v_{1}>=2380 \mathrm{~m} / \mathrm{s}\right.$ and $\delta v_{1}=350 \mathrm{~m} / \mathrm{s}$. It is seen that while the input velocity peaks at $2450 \mathrm{~m} / \mathrm{s}(1.0 \mathrm{eV}$ translational energy) [20], the output velocity peaks at about $2050 \mathrm{~m} / \mathrm{s}$. This shift due to the velocity dependent resolution function leads to the actual energy heing about $50 \%$ larger than the nominal energy. Because the resolution function severely distorts the calculated results, we will, 
after comparing the calculated results with the experimental measurements and establishing their credibility, present the undistorted calculated results. This will permit us to examine the calculation more closely and to see if any other lessons can be learned from it.

To determine the dependence of the calculated results on the initial translational velocity of Ar, we plot in figure 5 the laboratory differential scattering cross section as a function of the laboratory recoil velocity of $\mathrm{CsF}$ at the laboratory scattering angle of $60^{\circ}$. The dotted line is our impulse calculation for the Ar velocity $v_{1}=2450 \mathrm{~m} / \mathrm{s}$ and the CsF velocity $v_{2}=500 \mathrm{~m} / \mathrm{s}$ with the initial state of $C s F$ given by $v=1$ and $j=70$. The dashed line is the impulse calculation using the probability of the Ar velocity given by the formula $\mathrm{P}\left(\mathrm{v}_{1}\right)=\mathrm{v}_{1}{ }^{3} \exp \left(-\left[\left(\mathrm{v}_{1}-<\mathrm{v}_{1}>\right) / \delta \mathrm{v}_{1}\right]^{2}\right)$, while keeping the CsF velocity at $500 \mathrm{~m} / \mathrm{s}$. The experimental points are the same as in figure 1, taken from reference 3, and are normalized to match the calculation at the largest cross section. It is seen that the calculation is insensitive to averaging over the initial velocity distribution of Ar. Since most of the initial translational energy is supplied by Ar, it appears reasonable to assume that the calculation is also insensitive to averaging over the initial CsF velocity distribution. In the rest of the paper we will present the calculations for fixed velocities of the Ar and the CsF beams.

To investigate the variation of the calculated results with the initial vibrational quantum number of $\mathrm{CsF}$, we plot in figure 6 the laboratory differential scattering cross section as a function of the laboratory recoil velocity of $\mathrm{CsF}$ at the laboratory scattering angle of $60^{\circ}$. The velocity of the Ar beam is fixed at $v_{1}=2450 \mathrm{~m} / \mathrm{s}$ while that of the CsF beam is fixed at $v_{2}=500 \mathrm{~m} / \mathrm{s}$. The initial rotational level of $C s F$ is fixed at $j=60$. Solid curve, dotted and dashed curves are the results of our calculation for initial vibrational levels $v=1,2$, and 3 , respectively. The experimental points are the same as in figure 1 , taken from reference 3, and are normalized to match the calculation at the largest cross section. It is seen that the calculation is insensitive to the initial vibrational level of CsF. 
In the remainder of the paper we will assume that initial vibrational level of $\mathrm{CsF}$ is $\mathrm{v}=3$ because this is the average vibrational level at $1000^{\circ} \mathrm{K}$, the temperature of the $\mathrm{CsF}$ beam.

To investigate the dependence of the calculated differential cross sections on the initial rotational quantum number of $\mathrm{CsF}$ and to compare the calculated results with the experimentally measured ones, we plot in figure 7 the laboratory differential scattering cross section as a function of the laboratory recoil velocity of $\mathrm{CsF}$ at eight laboratory scattering angles: $25^{\circ}, 30^{\circ}, \ldots, 60^{\circ}$. The velocity of the Ar beam is fixed at $v_{1}=2450 \mathrm{~m} / \mathrm{s}$ while that of the $C s F$ beam is fixed at $v_{2}=500 \mathrm{~m} / \mathrm{s}$. The initial vibrational level of $C s F$ is fixed at $v=3$. Dotted, solid and dashed curves correspond to CsF initial rotational levels $j=30,60$, and 100 , respectively. The experimental points are taken from reference 3 , and are normalized at each angle separately, to match the calculation for $j=60$ at the largest cross section. It is seen that the calculation is sensitive to the initial rotational level of $\mathrm{CsF}$, and the best agreement for all eight laboratory scattering angles is given by the solid line, i.e., $\mathrm{j}=60$.

The calculated results are in excellent agreement with the measured ones at all of the eight available laboratory scattering angles. The calculated results are not very sensitive to the initial velocity distribution of the Ar beam or to the initial vibrational quantum number of the CsF beam. They are, however, sensitive to the initial rotational distribution of the $\mathrm{CsF}$ beam. The calculated results imply that the rotational distribution of the CsF beam peaks around $\mathrm{j}=60$, rather than $\mathrm{j}=30$ or $\mathrm{j}=100$. In other words, the rotational temperature of the beam, if our calculation is to be believed, is close to 1000 ${ }^{\circ} \mathrm{K}$, the temperature of the oven.

A word of caution here is, perhaps, in order. We are not saying that the magnitude of the ballistic peak in CsF-Ar system is independent of the initial relative translational energy, but that it is insensitive to whether one performs the calculation at the average initial relative translational energy of $1.0 \mathrm{eV}$ or averages the calculated differential cross sections over the experimental [1] Maxwell distribution centered at $1.0 \mathrm{eV}$. The 
magnitude of the ballistic peak does depend upon the initial relative velocity as is shoun by the fact that our model predicts a ballistic peak for the $I_{2}$ - $\operatorname{Ar}\left(I_{2}\right.$ beam temperature 250 ${ }^{\circ} \mathrm{K}$ ) at the initial relative translational energy of $0.12 \mathrm{eV}$ but not at $1.0 \mathrm{eV}$. Similarly, it is being stated that the magnitude of the ballistic peak is independent of the initial vibrational levc: of CsF only if that vibrational level is one of the first four levels

\section{DETAILED EXAMINATION OF THE CALCULATED RESULTS}

Because the resolution of the instrument distorts the calculated signal so severely, we present, in figure 8 , the calculated discrete spectra for $55^{\circ}$ and $25^{\circ}$ laboratory scattering angles. The initial rotational level of $\mathrm{CsF}$ for these calculations is $\mathrm{j}=60$; the remaining beam parameters are the same as in figure 7 , and the angle of the centroid velocity vector in the laboratory frame is $52.2^{\circ}$. The contribution to the ballistic peak at the laboratory scattering angle of $55^{\circ}$ is dominated by the resonant transfer of the relative translational energy into the internal motion, while at the laboratory scattering angle of $25^{\circ}$, it is determined by the kinematic rainbow, or the edge effect [11]. This is also the conclusion arrived at from figure 8 where, for the $55^{\circ}$ scattering the ballistic peak is centered at the recoil velocity equal to the velocity of the c.m. while, for the $25^{\circ}$ scattering the center of the ballistic peak is at a lower value of recoil velocity. The ballistic peak for the laboratory scattering of $55^{\circ}$ is centered at the c.m. velocity of 646 $\mathrm{m} / \mathrm{s}$ because, when all of the energy of relative translational motion has been resonantly transferred into internal energy, the molecule is stationary in the c.m. frame and it moves at the velocity of the c.m. in the laboratory frame. The molecules contributing to the ballistic peak at the $25^{\circ}$ scattering angle have a substantial c.m. recoil velocity, and here the ballistic peak arises because of the kinematic rainbow, or edge effect [11]. For this case the recoil velocity of the molecule in the c.m. frame is perpendicular to its recoil velocity in the laboratory frame, leading to the relationship $\left(v_{2}^{\prime}\right)^{2}=\left(v_{c m}\right)^{2}-\left(\omega_{2}^{\prime}\right)^{2}$. The 
laboratory recoil velocity, around which the ballistic peak is centered, is now smaller (about $575 \mathrm{~m} / \mathrm{s}$ ) than the velocity of the c.m.

It is worth noting that the ballistic peak for the $55^{\circ}$ laboratory scattering angle displays a profile similar to a Lorentzian. This is because the c.m. differential cross sections for the dominant transitions are, because of the density factor, proportional to the c.m. recoil velocity. The Jacobian varies inversely as the square of the c.m. recoil velocity. The laboratory differential cross sections are therefore inversely proportional to the c.m. recoil velocity (the Lorentzian profile would be inversly proportional to the square of the c.m. recoil velocity.)

The ballistic peak at the $25^{\circ}$ scattering angle has a more complicated structure than that at $55^{\circ}$. There are more transitions comprising the $25^{\circ}$ peak that have noticeably large cross sections. In addition, the transitions are not symmetrically placed around the central recoil velocity of $575 \mathrm{~m} / \mathrm{s}$. These observations can be understood by referring to figure 9 , which gives a plot of the absolute value of the Jacobian, for several laboratory scattering angles, versus the laboratory recoil velocity of $\mathrm{CsF}$ for a $0.25^{\circ}$ wide detector. When the laboratory scattering angle is equal to the laboratory angle of the centroid velocity $\left(52.2^{\circ}\right)$, the Jacobian is extremely large and very sharply peaked. As we move away from the direction of the centroid velocity, i.e., increase or decrease the laboratory scattering angle, the value of the Jacobian becomes smaller, the peak gets flatter, and the center of the peak moves towards smaller recoil velocities. This is because the farther we look from the direction of the c.m. velocity, the larger $\omega_{2}^{\prime}$ must be, and therefore the smaller $v_{2}^{\prime}$ must be so that the square of these two recoil velocities can be equal to the square of the centroid velocity. Because the Jacobian is much flatter at the $25^{\circ}$ scattering angle, the laboratory differential cross sections resemble the c.m. differential cross sections. These points are apparent in figure 10, which gives a plot of the c.m. differential cross section as a function of the laboratory recoil velocity of $\mathrm{CsF}^{F}$ at the laboratory scattering angles of $25^{\circ}$ and $50^{\circ}$. Since we are assuming an idcalized beam without lateral dimensions, 
each stick in the figure corresponds to a c.m. differential cross section at a definite c.m. scattering angle. Thefore, the c.m. differential cross sections in figure 10, upon multiplication with the appropriate Jacobian (figure 9) give the laboratory differential cross sections, plotted in figure 8. The c.m. differential cross sections resemble the laboratory differential cross sections for the $25^{\circ}$ scattering angle; for the $55^{\circ}$ scattering angle, on the other hand, the transitions most prominent in laboratory frame (figure8) are barely noticeable in the c.m. frame. It should also be noticed that the pseudoelastic peak shifts to smaller recoil velocities as the laboratory scattering angle moves away from the direction of the centroid velocity; the maximum intensity occurs at the recoil velocity of $960 \mathrm{~m} / \mathrm{s}$ for $25^{\circ}$ and at $1100 \mathrm{~m} / \mathrm{s}$ for $55^{\circ}$. This happens because, when the laboratory scattering angle is farther from the direction of the centroid velocity the angle between $w^{\prime} 2$ and $\mathrm{v}_{\mathrm{cm}}$ becomes closer to $90^{\circ}$, resulting in smaller value of $\mathrm{v}_{2}^{\prime}=\left[\left(\omega_{2}^{\prime}\right)^{2}+\left(\mathrm{v}_{\mathrm{cm}}\right)^{2}-2 \omega_{2}^{\prime} \cdot \mathrm{v}_{\mathrm{cm}}\right]^{\frac{1}{2}}$

A closer look at the details of the scattering at $25^{\circ}$ is provided by figure 11 , which is a plot, again for an idealized beam, of the c.m. differential cross section as a function of the laboratory recoil velocity of $\mathrm{CsF}$. The initial state of $\mathrm{CsF}$ is $v=3, j=60$; the Ar-CsF initial relative translational energy is $1.0 \mathrm{eV}$; the c.m. velocity is $646 \mathrm{~m} / \mathrm{s}$ at a laboratory angle of $52.2^{\circ}$. The three upper frames are the rotational transitions comprising the ballistic peak (Ar-F encounter) for final vibrational levels $v^{\prime}=2,3$ and 4; the three lower frames are the rotational transitions comprising the pseudoelastic peak (Ar-Cs encounter) for the same final vibrational levels. The rotational transitions comprising the ballistic peak have almost the same structure and appear to be independent of the final vibrational level. Between the recoil velocities of 200 and $400 \mathrm{~m} / \mathrm{s}$ there are supernumerary rainbows and a primary rainbow in each frame, and a secondary rainbow in the $v^{\prime}=4$ frame, the rotational quantum number increasing with increasing recoil velocity. On the right side of each frame, after a large gap in the center, the rotational 
quantum number decreases with increasing recoil velocity and the transitions appearing first, i.e., closest to $575 \mathrm{~m} / \mathrm{s}$ recoil velocity, are constrained by the kinematic rainbow-like singularity or the edge effect. The rotational transitions $j^{\prime}=194,189$, and 185 are the first to appear for the final vibrational quantum numbers 2,3 , and 4 , respectively. The highest final rotational level attainable is greater for smaller vibrational quantum numbers because more energy is available when the final vibrational quantum number is smaller. One can regard these rotational transitions as dynamical rainbows and state that kinematic rainbows lead to dynamic rainbows. As the recoil velocity increases and the final rotational quantum number decreases, the supernumerary, primary, and secondary rainbows are again seen. The differential cross sections comprising the ballistic peak exhibits a rich and complicated structure.

For the case of pseudoelastic scattering (Ar-Cs encounter) depicted in the lower three frames of figure 11 , there are at least two rotational rainbows in each frame. The frame corresponding to $v^{\prime}=2$ exhibits a secondary rainbow on the left while that for $v^{\prime}=4$ exhibits a secondary rainbow on the right. The smaller recoil velocities correspond to larger final rotational quantum numbers, while the larger recoil velocities correspond to smaller final rotational quantum numbers. The inelastic transitions move towards larger recoil velocities as more energy becomes available, i.e., as the final vibrational quantum number decreases. The limit of the change in the rotational quantum number is dictated by the conservation of angular momentum.

Figure 12 is a plot of the c.m. differential cross section as a function of the c.m. scattering angle. It is seen that when almost all of the initial relative translational energy has been converted into the internal energy, the c.m. differential cross section is independent of the c.m. scattering angle. This effect, which was predicted earlier [4], arises because, for the resonant energy transfer from translation to vibration-rotation, the final orbital motion has very small relative velocity and is therefore an isotropic s-wave. 
This is one of the rare instances in atom-diatoms collisions when one encounters partial waves with very low quantum numbers.

\section{CONCLUSIONS}

The basic question is, why do the experimental results agree with the calculated results, which are obtained by using a simplified atom-diatom potential'? Not only has the atom-diatom potential been approximated by the sum of two atom-atom potentials, the atom-atom potentials have in addition to this approximation, been replaced by two hard core potentials. A clue to the answer is provided by earlier work [18] which studied the deactivation of highly vibrationally excited $\mathrm{KBr}$ by $\mathrm{Ar}$ using exactly the same calculation. For the largest c.m. scattering angle $\left(75^{\circ}\right)$ for which the experimental measurements were available, the calculated c.m. differential cross section plotted as a function of the $\mathrm{KBr}$ c.m. recoil velocity agreed with the experimental measurements for all recoil velocities $(0-1000 \mathrm{~m} / \mathrm{s})$. For the smallest c.m. scattering angle $\left(45^{\circ}\right)$ for which the experimental measurements were available, on the other hand, the calculated c.m. differential cross section plotted as a function of the $\mathrm{KBr}$ c.m. recoil velocity agreed with the experimental measurements only for recoil velocities larger than about $700 \mathrm{~m} / \mathrm{s}$. The answer, then, lies in noting that: (i) the pseudoelastic peak, involving transfers of small amounts of energy between translation and rotation-vibration, corresponds to large c.m. scattering a.igles for which the attractive portion of the potential, in analogy with the $\mathrm{KBr}-\mathrm{Ar}$ results, makes no contribution, (ii) the ballistic peak involves transfer of a large fraction of the initial relative translational energy into internal energy, which can be caused, again in analogy with the $\mathrm{KBr}$-Ar results, only by the repulsive portion of the potential independent of the c.m. scattering angle and, (iii) the calculation is insensitive to the steepness of the repulsive portion of the potential. Preliminary work [23] with the exponential repulsive potential supports this conclusion. We have a situation where the two ends of the diatom scatter, independent of each other, from the repulsive part of the atom-atom interaction 
potential. Further, the scattering from the repulsive portion of the potential does not appear to strongly depend upon the steepness of the repulsive potential. This, we believe, is why this calculation works so well.

It was pointed out earlier that the Jacobian for the transformation from the c.m. to the laboratory frame may lead to the enhancement of the c.m. differential cross section by a factor of a million. This result is derived assuming an idealized beam of infinitesimal extent. For a beam with finite width and a detector of finite dimensions, the ballistic peak along the direction of the $\mathrm{c} . \mathrm{m}$. velocity will contain contributions from transitions which are not exactly resonant $T \rightarrow(V, R)$ processes. This may lead to an average value of the Jacobian which is considerably less than a million. The actual value will depend not only upon the extent of the two beams and the spread of their velocities, but also upon the molecule being studied. If the molecule has a large rotational constant and the energy spread of the beams is smaller than the spacing between the final rotational levels, the average Jacobian may still be close to a million. The parameters of the beams, the molecule sludied, and the dimensions of the detector will all have to be carefully considered for a more detailed answer.

Our results require that the $\mathrm{CsF}$ beam in the experiments [1-3] must not be rotationally relaxed. In fact, the observation of a ballistic peak for the CsI-Ar system and the non-observation of one for the $\mathrm{I}_{2}-\mathrm{Ar}$ system at the same relative translational energy $(\approx 1 \mathrm{eV})$ is explained by our model by postulating that transitions from higher rotational levels populated in the CsI beam (oven temperature $\approx 1000{ }^{\circ} \mathrm{K}$ ) lead to conversion of a much larger fraction of relative translational energy into internal energy than the low rotational levels available in the cooler (oven temperature $\approx 250^{\circ} \mathrm{K}$ ) $\mathrm{I}_{2}$ beam. This provides another test of our model.

Our calculations have been performed assuming idealized beams with infinitesimal extent. Still they agree remarkably well the experimental measurements. 
Perhaps it is because the broadening of the calculated results by the resolution function, discussed earlier, is much larger than that due to the finite extent of the colliding beams. It is also useful to point out that, since our calculation agrees so well with the experimental results for both the pseudoelastic peak and the ballistic peak, the earlier model of impulsive collisions [5], which certainly gives a much different value for the energy transferred during both the pseudoelastic and the ballistic collisions, may need to be reexamined.

Perhaps the most important result of this study is that the ballistic effect should be observable for all collision systems under appropriate circumstances.

This work was in part funded by AFOSR under task 2303EP and Phillips Laboratory project 007 . The authors are grateful to Dudley Herschbach for many interesting discussions and making available to them the relevant portions of the Ph.D. theses of Drs. Entemann, King, and Zare. 


\section{REFERENCES}

1. H.J. Loesch and D.R. Herschbach, "Ballistic Mechanisms for Vibrational and Rotational Theory in Ar + CsI Collisions," J. Chem. Phys. 57: 2038 (1972).

2. D.L. King, H.J. Loesch and D.R. Herschenbach, Discussions Faraday Soc. 55: II-34 (1973).

3. D.L. King, "Facile Four-Center and Six-Center Reactions," Ph.D. Thesis, Chemistry Department, Harvard University, 1974.

The scattering angles in this reference are measured from the direction of the Ar beam, whereas in references 1 and 2 they are measured from the direction of the cesium Halide beam. We, in this work, will follow references $i$ and 2 and measure the angles from the cesium Halide beam.

4. J.M. Sindoni and R.D. Sharma, "Mechanism of Ballistic Collisions," Phys. Rev. A 45: R2659 (1992).

J.M. Sindoni and R.D. Sharma, "Classical Model for Vibrational and Rotational Excitation of Diatomic Molecules by Collision," J. Chem. Phys. 97: 7377 (1992).

5. S.W. Benson, G.C. Bernard and James C. Wu, "Classical Model for Vibrational and Rotational Excitation of Diatomic Molecules by Collision," J. Chem. Phys. 38: 25 (1963).

See also S.W. Benson, The Foundations of Chemical Kinetics, McGraw-Hill Book Company, New York, NY, 1960.

6. R.D. Levine and R. B. Bernstein, Molecular Reaction Dynamics and Chemical Reactivity, Second Edition, oxford University Press, New York, 1987.

7. E. A. Entemann, "Reactive Scattering in Molecular Beams," Ph.D. Thesis, Chemistry Department, Harvard University, 1967.

8. T.T. Warnock and R.B. Bernstein, "Transformation Relationships from Center-of-Mass cross section and Excitation Functions to Observable Angular and Velocity Distributions of Scattered Flux," J. Chem. Phys. 49: 1878 (1968).

9. L.I. Schiff, Quantum Mechanics, McGraw-Hill Book Company, New York, 1968.

10. M.S. Child, Molecular Collision Theory, Academic Press, London, 1974. 
11. R.N. Zare, "Molecular Fluorescence and Photodissociation," Ph.D. Thesis, Chemistry Department, Harvard University, 1964 .

12. Molecular Collision Dynamics, Edited by J.M. Bowman, Springer-Verlag, New York, 1983, Chapter 4.

13. R.D. Sharma, P.M. Bakshi and J.M. Sindoni, "Impulse Formalism for Atom-Diatom Collisions," Phys. Rrv. A 43: 189 (1991).

14. J.M. Eisenberg and D.S. Kolton, Theory of Meson Interactions. with Nuclei, Wiley-Interscience Publication, John Wiley and Sons, New York, 1980.

15. A.S. Davydov, Quantum Mechanics, Addison Wesley Publishing Company, Reading, Massachusetts, U.S.A. (1964).

16. L.D. Landau and E.M. Lifshitz, Mechanics, Pergamon press, New York, 1969.

17. R.D. Sharma, P.M. Bakshi and J.M. Sindoni, "Criteria for Applicability of the Impulse Approach to Collisions," Phys. Rev. A (Rapid Communications;, 41: 6540 (1990).

18. R.D. Sharma and J.M. Sindoni, "Relaxation of Highly Vibrationally Excited KBr by Ar," Phys, Rev. A (Brief Reports) 45: 531 (1992).

19. M.E. Rose, Elementary Theory of Angular Momentum, John wiley and Sons, New York, 1957.

20. J.0. Hirschfelder, C.F. Curtiss and R. B. Bird, Molecular Theory of Gases and Liquids, John Wiley and Sons, New Yort: 1954 .

The hard core radii obtained were: $r\left(A r-\mathrm{Cs}^{+}\right)=3.8 \dot{A}$ and $r\left(A r-F^{-}\right)=3.1 A$. However, to conform with the earlier wort: [1] $\mathrm{r}\left(\mathrm{Ar}-\mathrm{CS}^{+}\right)$was changed to $3.5 \AA$.

21. K.P. Huber and G. Herzberg, Constants of Diatomic Molecules, Van Nostrand Reinhold Company, New York, 1979.

22. In reference [4], $v_{1}$, the velocity of Ar was taken equal to $2550 \mathrm{~m} / \mathrm{s}$; the relative translational energy corresponding to this velocity is $1.1 \mathrm{eV}$. It was since deemed more likely, based on the parameters in reference [3], that the actual beam velocity peaked at $2450 \mathrm{~m} / \mathrm{s}$, corresponding to relative translational energy of $1.0 \mathrm{eV}$.

23. H. Dothe and R. D. Sharma, "A Distorted Wave Impulse Approach for Atom-Diatom Collisions," (to be published). 


\section{FIGURE CAPTIONS}

Figure 1. Laboratory differential cross section as a function of the laboratory recoil velocity of $\mathrm{CsF}$ at the laboratory scattering angle of $60^{\circ}$. This figure is reproduced from reference 3 . The pseudoelastic peak, centered at the recoil velocity of about $1100 \mathrm{~m} / \mathrm{s}$, is where clastic scattering at large c.m. scattering angles (about $150^{\circ}$ ) should be observed. The peak at about $500 \mathrm{~m} / \mathrm{s}$ is the ballistic peak and is centered near the centroid velocity. The CsF molecules contributing to this peak are moving slowly in the c.m. frame.

Figure 2. Jacobian for the transformation of the differential cross section from the c.m. frame to the laboratory frame as a function of the final relative translational energy of CsF (measured as a fraction of the initial relative translational energy) when the scattering is viewed along the direction of the centroid velocity, i.e., $\theta_{2}=$ $\theta_{\mathrm{L}}$.

Figure 3. Calculated laboratory (c.m.) differential cross section $\left(\mathrm{cm}^{2} / \mathrm{sr}\right)$ as a function of laboratory (c.m.) scattering angle. Increased differential cross section at the minimum and maximum laboratory scattering angles is due to the kinematic rainbows, also called edge effect [11], and not due to any peculiarities in the c.m. differential cross section. The collision parameters are the same as in figure $3 \mathrm{~A}$.

Figure 4. Flux of the Ar parent beam, in arbitrary units, as a function of the velocity of Ar. The input function (solid line) represents the actual velocity distribution and is given by $P\left(v_{1}\right)=v_{1}{ }^{3} \exp \left(-\left[\left(v_{1}-<v_{1}>\right) / \delta v_{1}\right]^{2}\right)$ with $<v_{1}>=2380 \mathrm{~m} / \mathrm{s}$ and $\delta v_{1}=350 \mathrm{~m} / \mathrm{s}$. The output velocity distribution (dotted line), represents the measured Ar parent beam flux and is obtained by modifying the input distribution by 
a normalized Gaussian function with full width at half height given by the resolution function $R$. The actual velocity distribution peaks at $2450 \mathrm{~m} / \mathrm{s}$ but the measured peak is at about $2050 \mathrm{~m} / \mathrm{s}$. The energy associated with the output distribution peak is about $71 \%$ of the energy corresponding to the maximum of the input distribution.

Figure 5. Laboratory differential cross section as a function of the laboratory recoil velocity of $\mathrm{CsF}$ at the laboratory scattering angle of $60^{\circ}$. Dotted line is the result of the impulse calculation for Ar velocity $v_{1}=2450 \mathrm{~m} / \mathrm{s}$ and CsF velocity $v_{2}=500 \mathrm{~m}$ 's with the initial state of $\mathrm{CsF}$ given by $v=1$ and $j=70$. The dashed line is the result of the impulse calculation obtained by averaging over the Ar velocity according to the distribution given in reference 1; the velocity of the CsF beam is still $500 \mathrm{~m} / \mathrm{s}$. The experimental points are the same as in figure 1 , taken from reference 3 , and are normalized to match the calculation for discrete velocity at the largest cross section.

Figure 6. Laboratory differential cross section as a function of the laboratory recoil velocity of $\mathrm{CsF}$ at the laboratory scattering angle of $60^{\circ}$. The velocity of the $\mathrm{Ar}$ and the CsF beams are $v_{1}=2450 \mathrm{~m} / \mathrm{s}$ and $v_{2}=500 \mathrm{~m} / \mathrm{s}$ with the initial rotational state of CsF fixed at $j=60$. The impulse calculations for initial vibrational levels $v=1,2$ and 3 are represented by solid, dotted and dashed lines, respectively.

Figure 7. Laboratory differential cross section as a function of the laboratory recoil velocity of $\mathrm{CsF}$ at eight laboratory scattering angles $25^{\circ}-60^{\circ}$. The velocity of the $\mathrm{Ar}$ and the CsF beams are $v_{1}=2450 \mathrm{~m} / \mathrm{s}$ and $v_{2}=500 \mathrm{~m} / \mathrm{s}$ with the initial vibrational state of $\mathrm{CsF}$ fixed at $\mathrm{v}=3$. The impulse calculations for initial rotational levels $\mathrm{j}=60,100$ and 30 are represented by solid, broken and dotted lines, respectively. 
Figure 8. Calculated laboratory differential cross section as a function of the laboratory recoil velocity of $\mathrm{CsF}$ for the laboratory scattering angles of $25^{\circ}$ and $55^{\circ}$ for initial vibrational-rotational level $(v=3, j=60)$ of $\mathrm{CsF}$. The remainder of the beam parameters are the same as in figure 7.

Figure 9. Absolute value of the Jacobian for the transformation of the differential cross section from the c.m. coordinate system to the laboratory coordinate system as a function of the laboratory recoil velocity of $\mathrm{CsF}$ for various laboratory scattering angles.

Figure 10. Center-of-mass differential cross section $\left(\mathrm{cm}^{2} / \mathrm{sr}\right)$ as a function of the laboratory recoil velocity $(\mathrm{m} / \mathrm{s})$ of $\mathrm{CsF}$ at the laboratory scattering angles of $25^{\circ}$ (top frame) and $55^{\circ}$ (bottom frame). The beam parameters are the same as in figure 8 .

Figure 11. Center-of-mass differential cross section $\left(\mathrm{cm}^{2} / \mathrm{sr}\right)$ as a function of the laboratory recoil velocity $(\mathrm{m} / \mathrm{s})$ of $\mathrm{CsF}$ at the laboratory scattering angle of $25^{\circ}$, for the most prominent transitions making up the ballistic peak (top three frames), and the most prominent transitions making up the pseudoelastic peak (bottom three frames). The beam parameters are same as in figure 8 .

Figure 12. Calculated c.m. differential cross section as a function of the c.m. scattering angle. When almost all of the initial relative translational energy has been converted into internal motion, the final relative translational energy carries no angular momentum, leading to isotropic scattering. 


\section{APPENDIX}

To derive the expression for the Jacobian of transformation between the laboratory and the center of mass coordinates as shown in figure $1 \mathrm{~A}$, we pick the following notation:

(i) $\mathrm{v}_{1}$, the initial velocity of Ar atoms, is along the X-axis in the laboratory coordinate system

(ii) $v_{2}$, the initial velocity of the alkali halide, is along the $\mathrm{Z}$-axis in the laboratory coordinate system

(iii) The coordinate system fixed in the laboratory frame is denoted by XYZ while that fixed in the c.m. frame is denoted by xyz.

The initial relative velocity and the velocity of the c.m. are, obviously, in the $\mathrm{XZ}$ plane in the laboratory coordinate system. We also take this plane to be the $\mathrm{xz}$ plane of the c.m. coordinate system. In addition, we assume that the detector is also centered in this plane. $\theta_{\mathrm{L}}$ and $\theta_{2}$ are the polar angles of the alkali halide recoil velocity $v_{2} 2$ and of the centroid velocity $v_{c . m}$, respectively, in the laboratory coordinate system. $\theta_{1}$ is the angle between the initial velocity $\omega_{2}$ of the alkali halide in the c.m. frame and the centroid velocity $\mathbf{v}_{\text {c.m. }}$. $\theta$ (the scattering angle) is the angle between the c.m. initial $\left(\omega_{2}\right)$ and c.m. final (recoil, $\left.\omega_{2}^{\prime}\right)$ velocities of the alkali halide and $\phi$ is the azimuthal angle of $\omega_{2}^{\prime}$, measured from the $x z$ plane. It should be pointed out that while the differential solid angle in the laboratory fixed axis is $d \Omega_{L}=\sin \theta_{L} d \theta_{L} d \phi_{L}$, the element of solid angle in the c.m. frame is $d \Omega=\sin \left(\theta-\theta_{1}+\theta_{2}\right) d \theta d \phi$. The vector equation

$$
\mathbf{v}_{2}^{\prime}=v_{\text {c.m. }}+\omega_{2}^{\prime}
$$

can be rewritten as 
$\gamma_{1} \cos \theta_{L}=\gamma \cos \theta_{2}+\cos \left(\theta-\theta_{1}+\theta_{2}\right)$

$\gamma_{1} \sin \theta_{L} \cos \phi_{L}=\gamma \sin \theta_{2}+\sin \left(\theta-\theta_{1}+\theta_{2}\right) \cos \phi$

$\gamma_{1} \sin \theta_{L} \sin \phi_{L}=\sin \left(\theta-\theta_{1}+\theta_{2}\right) \sin \phi$,

where $\gamma \equiv \frac{\mathrm{v}_{\mathrm{cm}}}{\omega_{2}^{\prime}}$ and $\gamma_{1} \equiv \frac{\mathrm{v}_{2}^{\prime}}{\omega_{2}^{\prime}}$. From equations (2A) we get

$$
\tan \phi_{L}=\frac{\sin \phi}{\left(\frac{\gamma \sin \theta_{2}}{\sin \left(\theta-\theta_{1}+\theta_{2}\right)}+\cos \phi\right)},
$$

yielding

$$
\sin \phi_{\mathrm{L}}=\frac{\sin \phi}{\left(1+\gamma^{2} \frac{\sin ^{2} \theta_{2}}{\sin ^{2}\left(\theta-\theta_{1}+\theta_{2}\right)}+2 \gamma \frac{\sin \theta_{2} \cos \phi}{\sin \left(\theta-\theta_{1}+\theta_{2}\right)}\right)^{\frac{1}{2}}} .
$$

Differentiating this equation, we get

$$
\left|\frac{d \phi_{L}}{d \phi}\right|=\left|1+\frac{\gamma \sin \theta_{2} \cos \phi}{\sin \left(\theta-\theta_{1}+\theta_{2}\right)}\right| .
$$

We now recall that our detector is centered in the $X Z$ plane. This enables us to put $\phi=0$ in the above equation obtaining

$$
\begin{aligned}
\left|\frac{d \phi_{\mathrm{L}}}{\mathrm{d} \phi}\right|^{-1} & =\left|1+\gamma \frac{\sin \theta_{2}}{\sin \left(\theta-\theta_{1}+\theta_{2}\right)}\right| \\
& =\left|\frac{\gamma_{1} \sin \theta_{1}}{\sin \left(\theta-\theta_{1}+\theta_{2}\right)}\right| .
\end{aligned}
$$


To obtain the relation between $d \theta$ and $d \theta_{l}$, we rewritc equations ( $\left.2 A\right)$ for $\phi=0$,

$$
\begin{aligned}
& \gamma_{1} \cos \theta_{L}=\gamma \cos \theta_{2}+\cos \left(\theta-\theta_{1}+\theta_{2}\right) \\
& \gamma_{1} \sin \theta_{L}=\gamma \sin \theta_{2}+\sin \left(\theta-\theta_{1}+\theta_{2}\right)
\end{aligned}
$$

Squaring the above equations and adding gives

$$
\gamma_{1}^{2}=\gamma^{2}+1+2 \gamma \cos \left(\theta-\theta_{1}\right)
$$

Dividing the second equation $(5 \mathrm{~A})$ by the first one, we get

$$
\tan \theta_{L}=\frac{\gamma \sin \theta_{2}+\sin \left(\theta-\theta_{1}+\theta_{2}\right)}{\gamma \cos \theta_{2}+\cos \left(\theta-\theta_{1}+\theta_{2}\right)},
$$

resulting in

$$
\cos \theta_{L}=\frac{\gamma \cos \theta_{2}+\cos \left(\theta-\theta_{1}+\theta_{2}\right)}{\left[1+\gamma^{2}+2 \gamma \cos \left(\theta-\theta_{1}\right)\right]^{\frac{1}{2}}}
$$

Differentiating,

$$
\begin{aligned}
\left|\frac{d \theta_{L}}{d \theta}\right|^{-1} & =\left|\sin \theta_{1}\right|\left(1+\gamma^{2}+2 \gamma \cos \left(\theta-\theta_{1}\right)\right)^{\frac{3}{2}} \\
& \times\left|\left\{\gamma^{2} \cos \left(\theta-\theta_{1}\right) \sin \theta_{2}+\sin \left(\theta-\theta_{1}+\theta_{2}\right)+\gamma \sin \theta_{2}+\gamma \sin \left(\theta-\theta_{1}+\theta_{2}\right) \cos \left(\theta-\theta_{1}\right)\right\}\right|^{\prime} \\
& =\frac{\gamma_{1}^{2}}{\left|1+\gamma \cos \left(\theta-\theta_{1}\right)\right|}=\frac{2 \gamma_{1}^{2}}{\left|1+\gamma_{1}^{2}-\gamma^{2}\right|}=\frac{\gamma_{1}}{\left|\hat{\omega}_{2}^{\prime} \cdot \hat{v}_{2}^{\prime}\right|},
\end{aligned}
$$

giving us

$$
\begin{aligned}
\left|\frac{d \Omega_{1}\left(\theta_{1}, \phi_{1}\right)}{d \Omega(\theta, \phi)}\right|^{\prime} & =\frac{2 \gamma_{1}^{3}}{\left|1+\gamma_{1}^{2}-\gamma^{2}\right|} \\
& =\frac{\gamma_{1}^{2}}{\left|\left(\hat{\omega}_{2}^{\prime} \cdot \hat{v}_{2}^{\prime}\right)\right|},
\end{aligned}
$$


where $\left(\hat{\omega}_{2}^{\prime} . \hat{v}_{2}^{\prime}\right)$ is the cosine of the angle between the recoil velocitics of particle $2(C s X)$ in the c.m. and the laboratory frames, and the relation $\gamma^{2}=1+\gamma_{1}^{2}+2 \cos \left(\hat{\omega}_{2}^{\prime} \cdot \hat{v}_{2}^{\prime}\right)$ has been used to obtain the last form. The Jacobian, as was pointed out earlier, becomes infinite when either the recoil velocity of the alkali halide in the center-of mass frame $\omega_{2}^{\prime}$ becomes very small, or when the recoil velocities of the alkali halide in the c.m. and the laboratory frames are perpendicular. Since the Jacobian, a transformation between two finite differential cross sections, cannot physically become infinite, a separate expression for it must be derived for the two cases cited above.

When one of the collision partners is initially stationary in the laboratory frame, i.e., $v_{1}=0$ and $\theta_{1}=\theta_{2}=0$, this expression reduces to the one given $b y$ Sc'iff [9].

$$
\left|\frac{d \Omega_{L}\left(\theta_{L}, \phi_{L}\right)}{d \Omega(\theta, \phi)}\right|^{\prime}=\left(\frac{\left(1+\gamma^{2}+2 \gamma \cos \theta\right)^{2}}{|1+\gamma \cos \theta|}\right)=\frac{2 \gamma_{1}^{3}}{\left|1+\gamma_{1}^{2}-\gamma^{2}\right|}
$$

Figure $2 \mathrm{~A}$ shows that a maximum (top figure) and a minimum (bottom figure) laboratory scattering angle exists when the recoil velocities of the alkali halide in the c.m. and the laboratory frames are perpendicular. This is further clarified in figure $3 \mathrm{~A}$ which gives a plot of the c.m. scattering angle as a function of the laboratory scattering angle. This figure clearly displays the extrema in the laboratory scattering angle as a function of the c.m. scattering angle. At these extrema the expression $8 \mathrm{~A}$ for the Jacobian becomes infinite. Figure $4 \mathrm{~A}$ gives a plot of the laboratory recoil velocity of $\mathrm{CsF}$ as a function of the laboratory scattering angle. The laboratory recoil velocity displays an extremum at the minimum and maximum laboratory scattering angles. This is the reason that the absolute value of the Jacobian stays constant for large changes in the laboratory recoil velocity of CsF (figure 9). To derive an expression for the Jacobian when the recuil 
velocities of the alkali halide in the c.m. and the laboratory frames are perpendicular, one can write, using figure $5 \mathrm{~A}$,

$$
\begin{aligned}
\cos \left(\frac{\Delta \theta}{2}\right) & =\left(\frac{\omega_{2}^{\prime}-v_{2}^{\prime} \Delta \theta_{L}}{\omega_{2}^{\prime}}\right) \\
& \approx\left(1-\gamma_{1} \Delta \theta_{L}\right)
\end{aligned}
$$

or,

$$
\Delta \theta \approx 2 \arccos \left(1-\gamma_{1} \Delta \theta_{2}\right)
$$

Using the relations

$\arcsin (x)+\arccos (x)=\pi / 2$, and,

$\arcsin (1-x) \approx \frac{\pi}{2}-(2 x)^{2}$, we get

$\left|\frac{\Delta \theta_{L}}{\Delta \theta}\right|^{-1} \approx\left|\frac{8 \gamma_{1}}{\Delta \theta_{L}}\right|^{\frac{1}{2}}$

Together with

$$
\left|\frac{d \phi_{L}}{d \phi}\right|^{-1}=\gamma_{1}\left|\frac{\sin \theta_{1}}{\sin \left(\theta-\theta_{1}+\theta_{2}\right)}\right|,
$$

and

$$
d \Omega=\sin \left(\theta-\theta_{1}+\theta_{2}\right) d \theta d \phi,
$$

and

$$
d \Omega_{L}=\sin \theta_{L} d \theta_{L} d \phi_{L}
$$

we get,

$$
\left|\frac{d \Omega_{L}}{d \Omega}\right| \approx\left|\frac{8 \gamma_{1}^{3}}{\Delta \theta_{L}}\right|^{\frac{1}{2}}
$$


When the laboratory recoil velocity is along the centroid velocity, one can write, using figure (1A), $\theta=\theta_{1}$, and $\theta_{2}=\theta_{\mathrm{L}}$, giving

$$
\left|\frac{d \Omega_{L}}{d \Omega}\right|^{-1}=\gamma_{1}^{2}
$$

This expression for the Jacobian is valid only when the laboratory recoil velocity is along the centroid velocity provided $\gamma_{1} \Delta \theta_{\mathrm{L}} \leq 1$ and $\gamma_{1} \Delta \phi_{\mathrm{L}} \leq 1, \Delta \theta_{\mathrm{L}}$ and $\Delta \phi_{\mathrm{L}}$ being the width of the laboratory detector in the polar and azimuthal angles. When this condition does not hold, the Jacobian becomes a constant and is simply equal to $\frac{4 \pi}{\Delta \Omega_{L}}$. This is because the signal from all $4 \pi$ steradians in the $c . m$. frame is now collected by the detector; a wider detector cannot collect any more signal.

To make the connection with the previous work $[2,3]$, we start with equation $(8 \mathrm{~A})$ and note that

$$
\hat{\omega}_{2}^{\prime} \cdot \hat{v}_{2}^{\prime}=\frac{d \omega_{2}^{\prime}}{d v_{2}^{\prime}}
$$

This equation is easy to prove. Using figure (1A), we write

$$
\left(\omega_{2}^{\prime}\right)^{2}=\left(v_{2}^{\prime}\right)^{2}+v_{c m}^{2}-2 v_{2}^{\prime} v_{c m} \cos \left(\theta_{1}-\theta_{2}\right)
$$

Differentiating equation (16A), we get

$$
\omega_{2}^{\prime} d \omega_{2}^{\prime}=v_{2}^{\prime} d v_{2}^{\prime}-v_{c m} d v_{2}^{\prime} \cos \left(\theta_{1}-\theta_{2}\right)
$$

Substituting for $\cos \left(\theta_{\mathrm{L}}-\theta_{2}\right.$ ) from equation (16A) we get equation (15A). Going back to equation (8A), when there is a continuous distribution of energy levels, the Jacobian 
becomes just $\gamma_{1}^{2}$. In our case, since we are dealing with discrete quantized rotationalvibrational levels, the Jacobian is given by equation $(8 \mathrm{~A})$. 


\section{FIGURE CAPTIONS (APPENDIX)}

Figure 1A. Vector diagram for the CsF-Ar scattering. The initial laboratory velocity of the Ar beam $\left(v_{1}\right)$ is measured along the $X$-axis and that of the $\operatorname{CsF}\left(v_{2}\right)$ beam along the $Z$ axis. The laboratory angles are measured from the direction of the CsF beam; $\theta_{2}$ and $\theta_{L}$ are the laboratory angles of the centroid velocity $\mathbf{v}_{c . m}$. and the recoil velocity $\mathbf{v}_{2}^{\prime}$ (laboratory scattering angle), respectively. The c.m. scattering angle is measured from $\mathrm{CB}$, the direction of initial c.m. velocity of $\operatorname{CsF}\left(\omega_{2}\right)$. The pseudoelastic peak discussed in this article, centered at a laboratory recoil velocity of about $1000 \mathrm{~m} / \mathrm{s}$, arises from the larger c.m. scattering angles. The other pseudoelastic peak, centered at a laboratory recoil velocity of about $150 \mathrm{~m} / \mathrm{s}$, arising from smaller c.m. scattering angles, was not experimentally detected and will not be further discussed. The circle in the figure corresponds to one-half of the initial relative translational energy being converted into internal energy.

Figure 2A. Vector diagrams to demonstrate the existence of maximum (top figure) and minimum (bottom figure) laboratory scattering angles for a given value of the c.m. recoil velocity $\left(\omega_{2}^{\prime}\right)$ of CsF. At the maximum and minimum laboratory scattering angles, the c.m. and laboratory recoil velocities are perpendicular. The extrema in the laboratory scattering angle, as a function of the c.m. scattering angle, lead to rainbow like singularities in the Jacobian for the c.m. to laboratory transformation at these points.

Figure 3A. Plot of the laboratory scattering angle $\theta_{\mathrm{L}}$ as a function of the center of mass scattering angle $\theta$ for the $\left(v=3, j=80 \quad->v^{\prime}=20, j^{\prime}=118\right)$ transition of CsF $\left(v_{1}=2450 \mathrm{~m} / \mathrm{s}\right.$, $v_{2}=500 \mathrm{~m} / \mathrm{s}, \Delta E / E=0.7778, \omega_{2}^{\prime}=246 \mathrm{~m} / \mathrm{s}, v_{c . m}=646 \mathrm{~m} / \mathrm{s}$ ). It is seen that the laboratory scattering angle, for these collision parameters, cannot be less than about $30^{\circ}$ and more than about $75^{\circ}$. The laboratory recoil velocity $v_{2}^{\prime}$ and the c.m. recoil velocity $\omega_{2}^{\prime}$ are 
perpendicular for the maximum and minimum laboratory scattering angles and the laboratory scattering angle as a function of c.m. scattering angle displays an extremum. This leads to the display of rainbows in the curve of the laboratory differential cross section as a function of the laboratory recoil velocity at the maximum and minimum laboratory scattering angles. The discontinuities in the curve at c.m. scattering angles of $0^{\circ}$ and $180^{\circ}$ are not real and arise from the fact that the c.m. scattering angle is measured modulo $\pi$ and not modulo $2 \pi$.

Figure 4A. Laboratory recoil velocity of $\mathrm{CsF}$ as a function of the laboratory scattering angle. The collision parameters are the same as in figure $3 \mathrm{~A}$. At the rainbow angles, $v^{2}$ c.m. $=\left(\omega_{2}^{\prime}\right)^{2}+\left(v_{2}^{\prime}\right)^{2}$ and the laboratory recoil velocity of CsF is $597 \mathrm{~m}$ 's, slightly smaller than the velocity of the center of mass. When the laboratory scattering angle is not equal to the rainbow angles, the signal from each transition is observed at two recoil velocities, one lower and one higher than the velocity of the center of mass, corresponding to the cases a and $b$ center of mass scattering angles. Both cases contribute to the ballistic peak.

Figure 5A. Vector diagram illustrating the uncertainty in the c.m. scattering angle due to a small uncertainty in the laboratory scattering angle at the rainbow angle. The angular resolution of the detector, $\Delta \theta_{\mathrm{L}}$, is exaggerated for the purpose of clarity. 


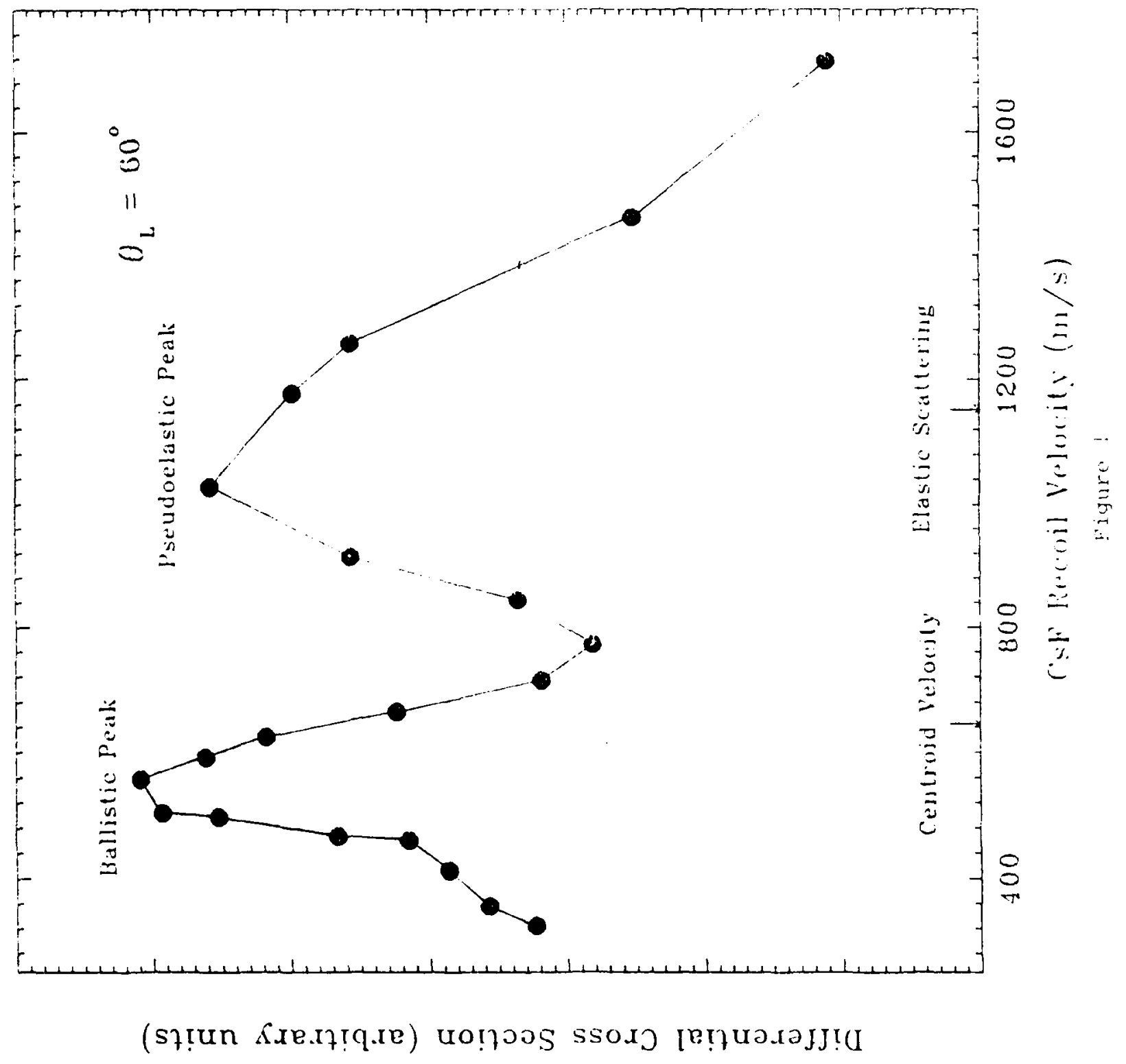




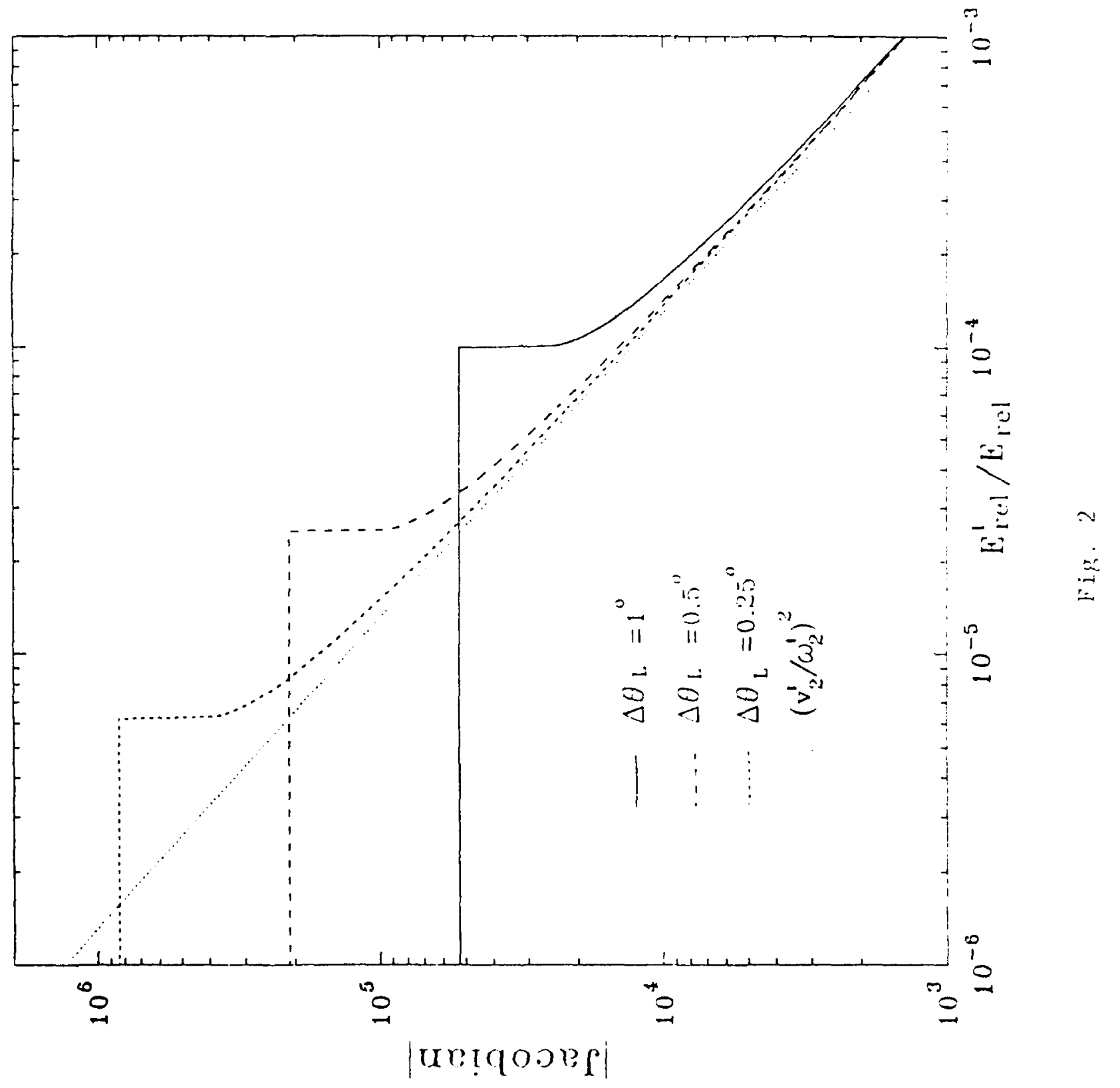



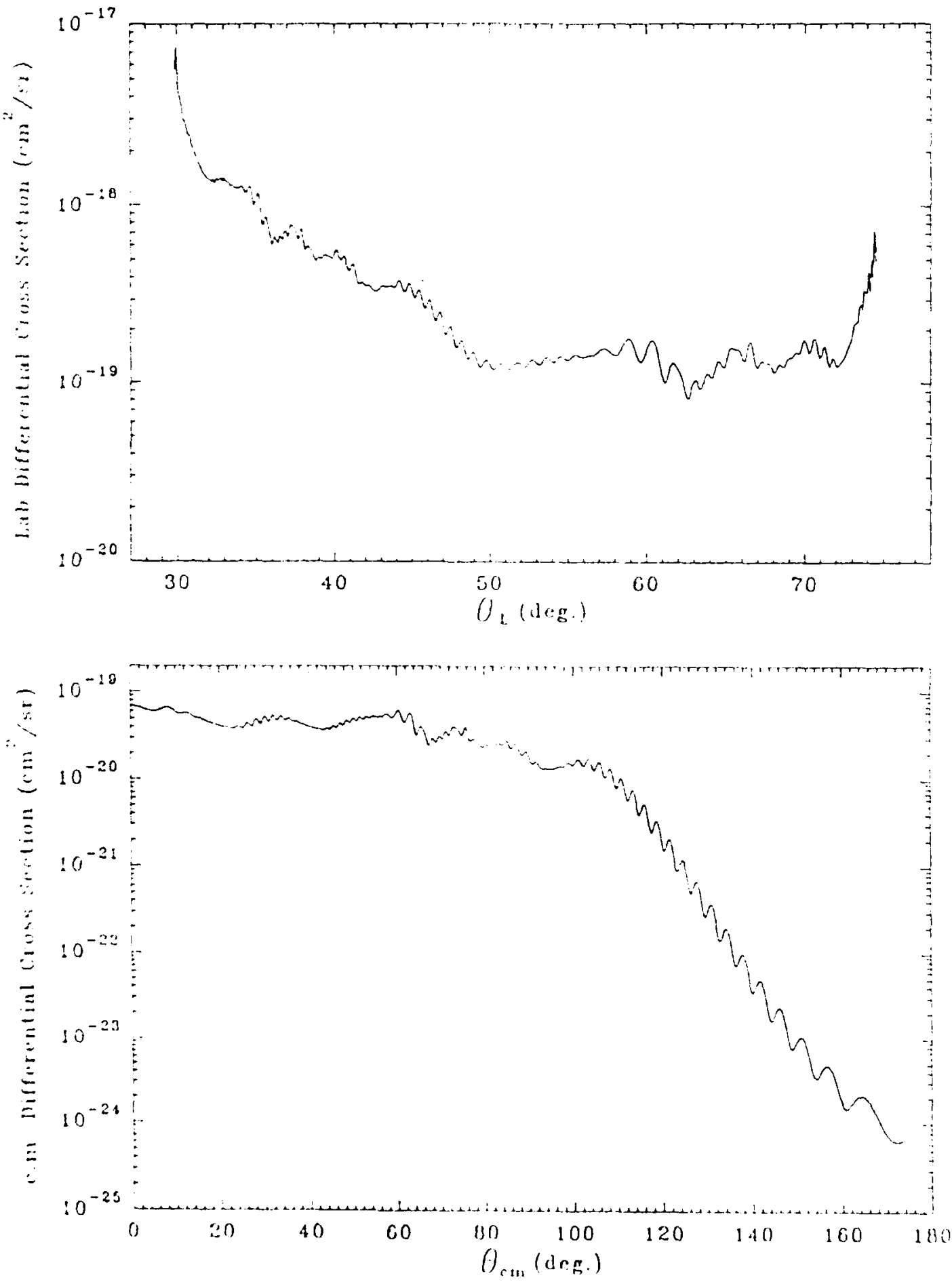


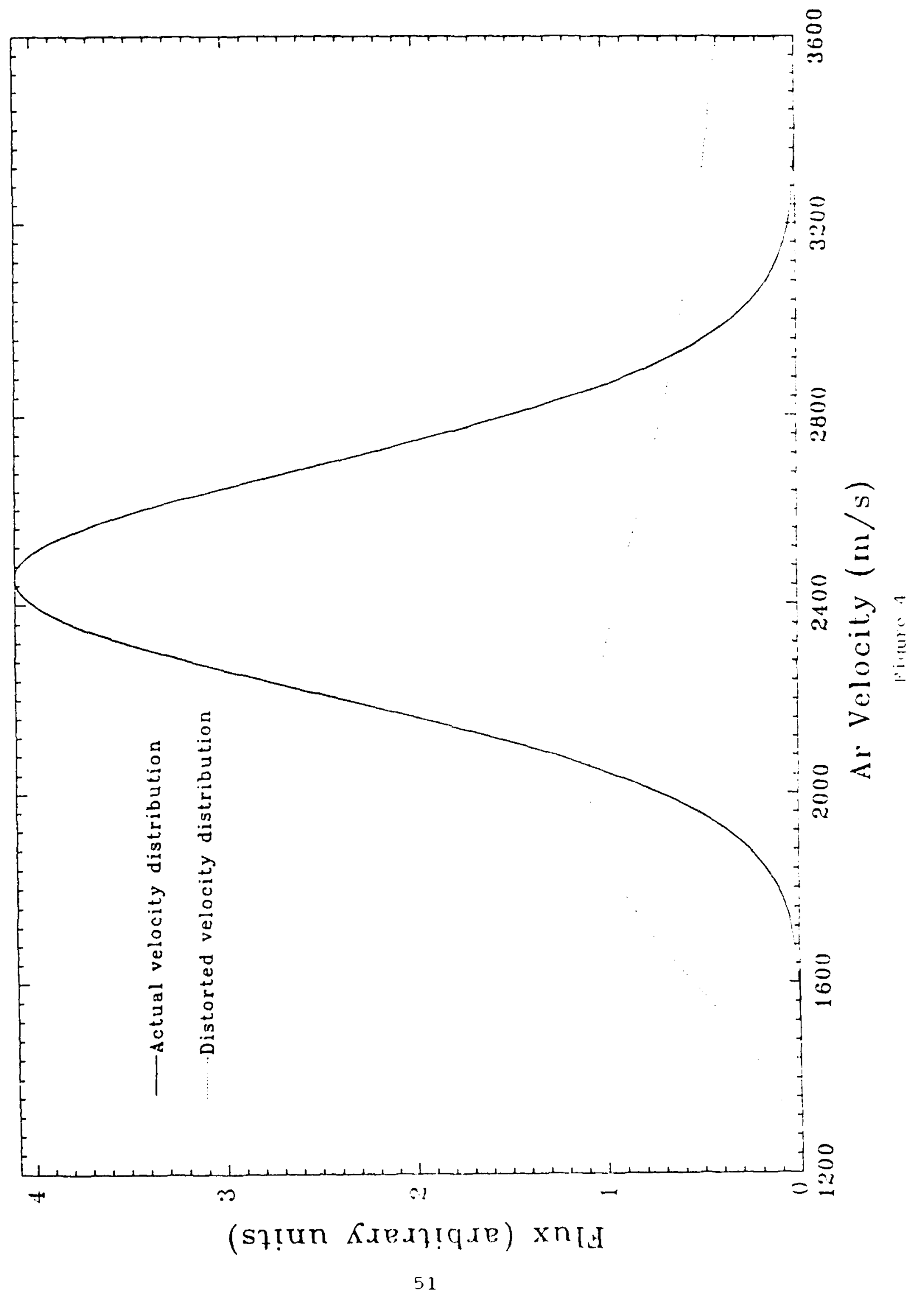




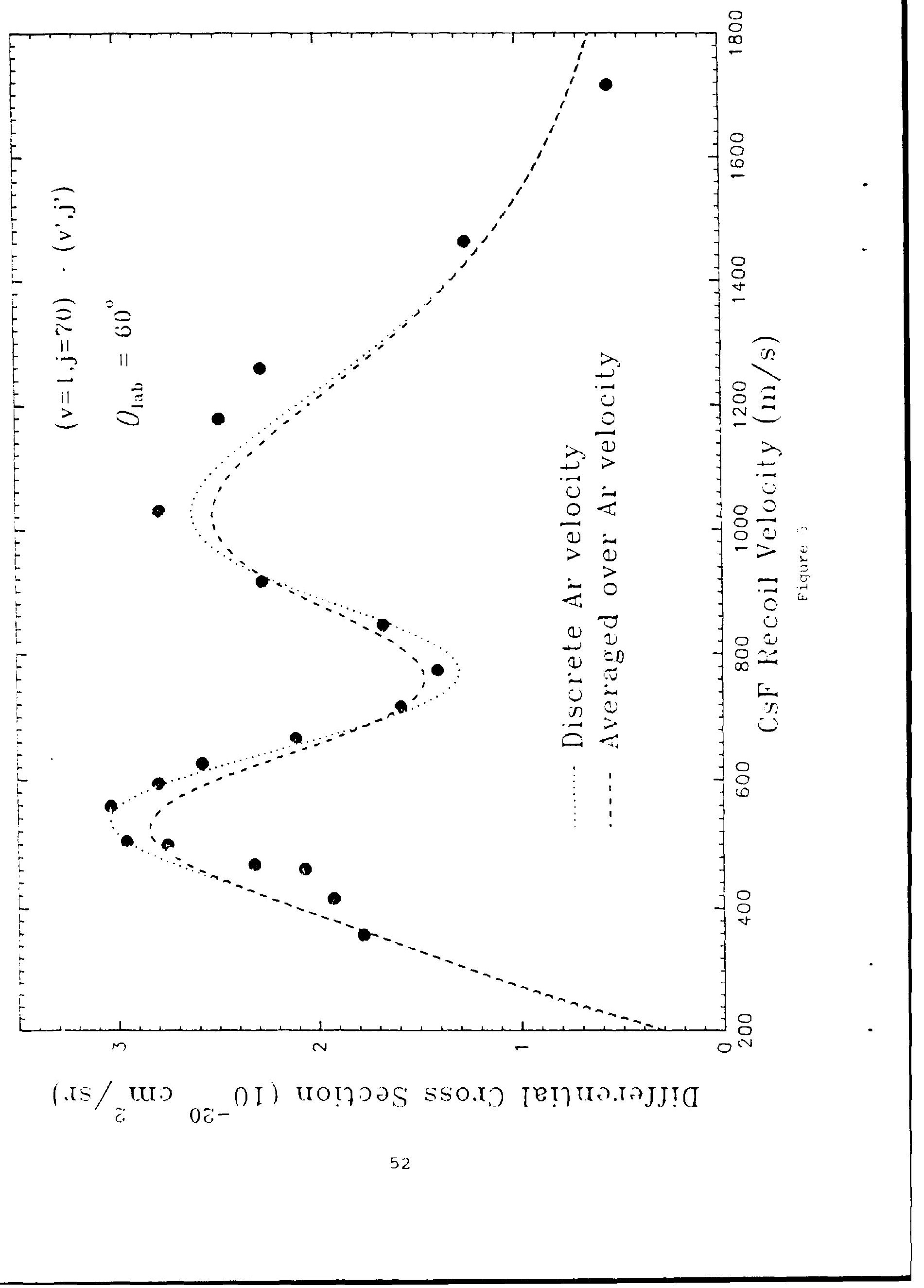




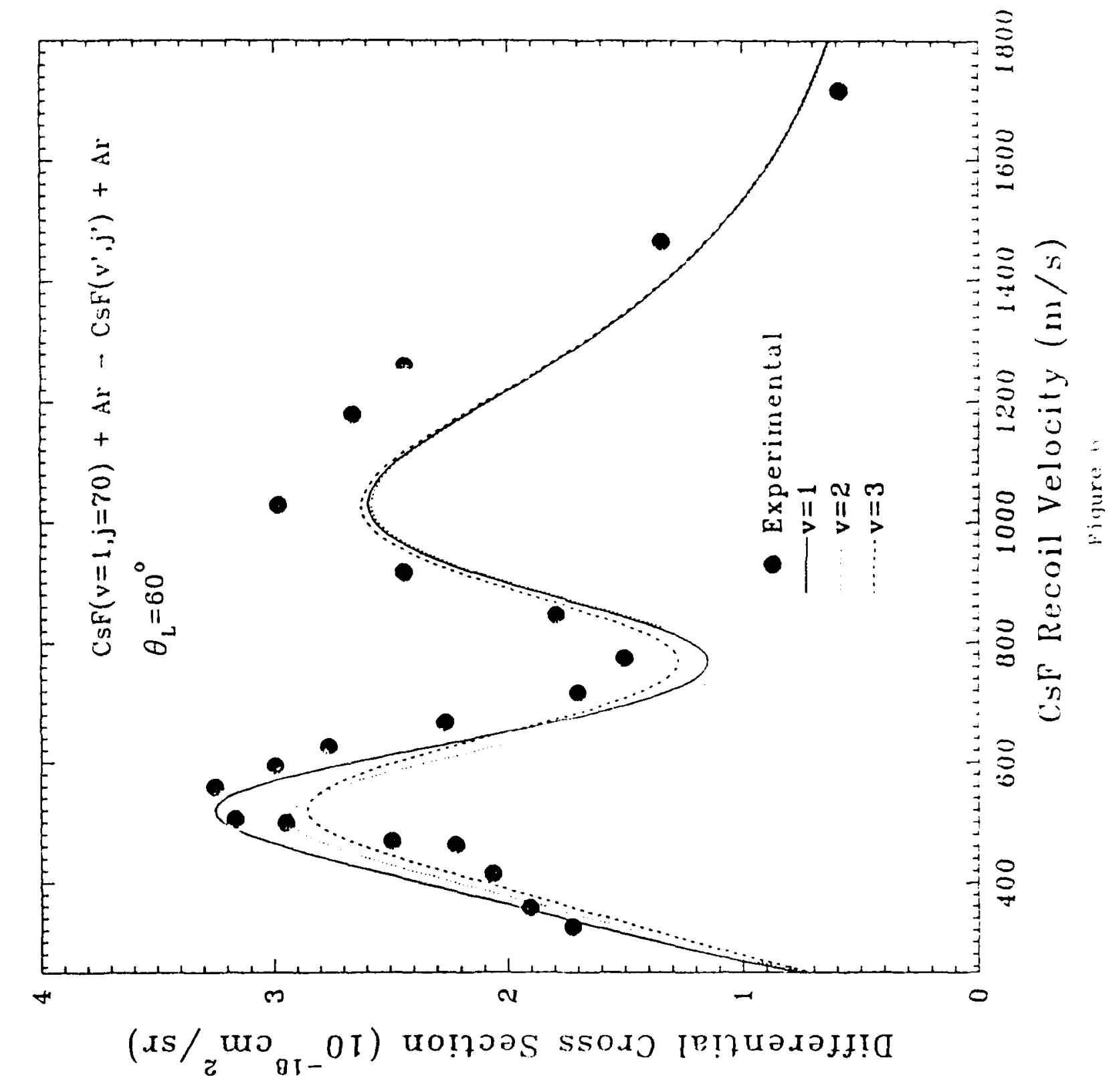




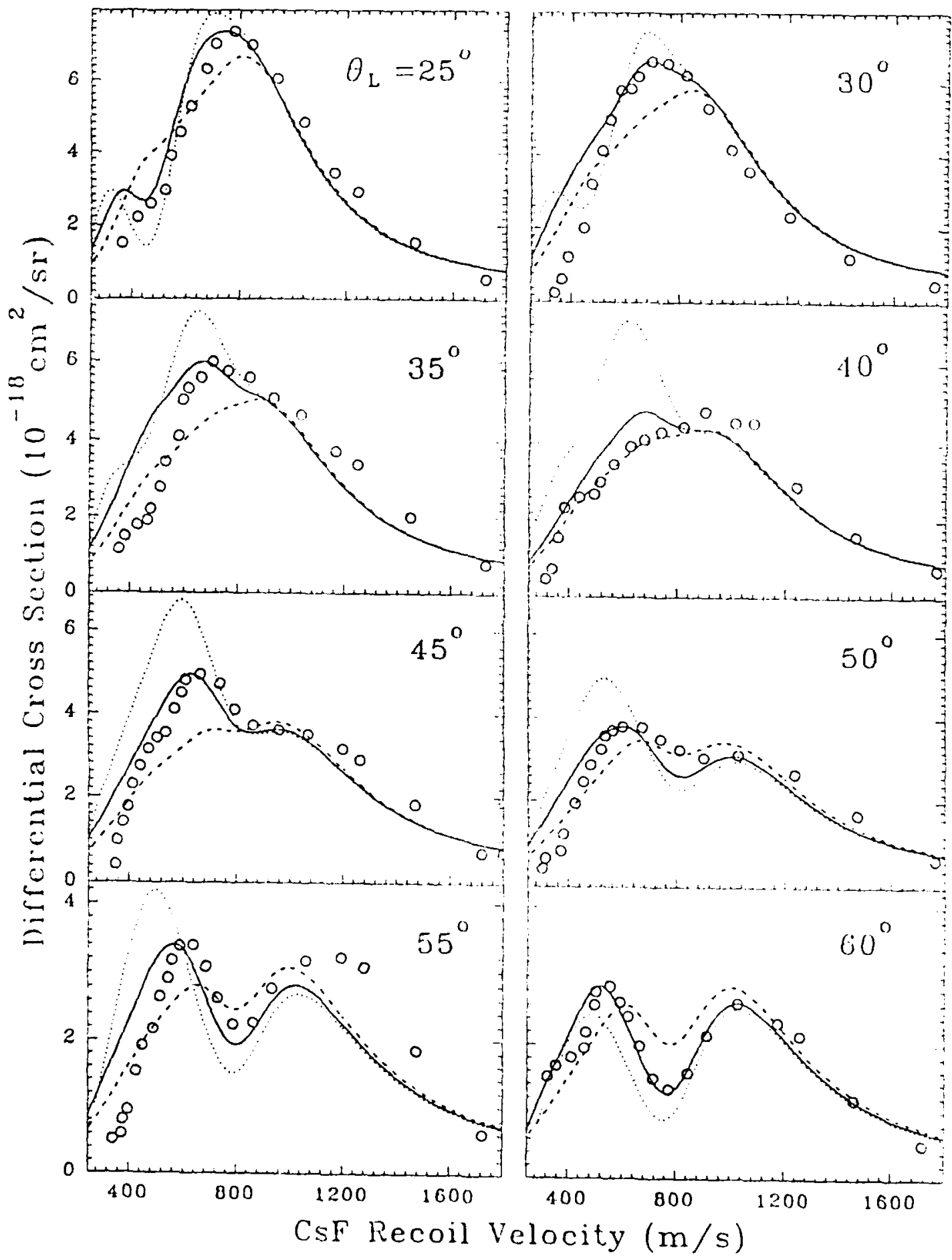




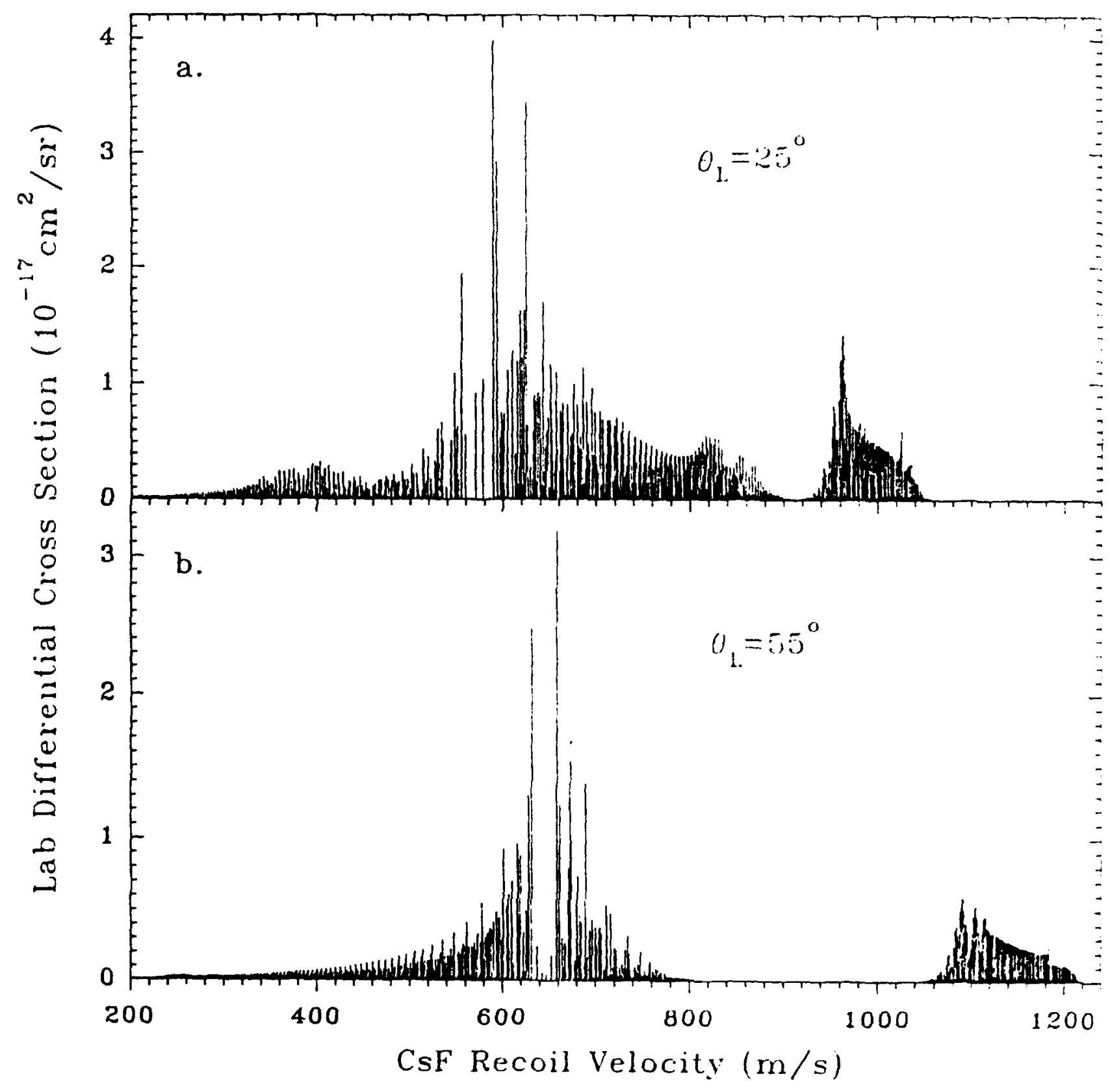

1. I r r : 


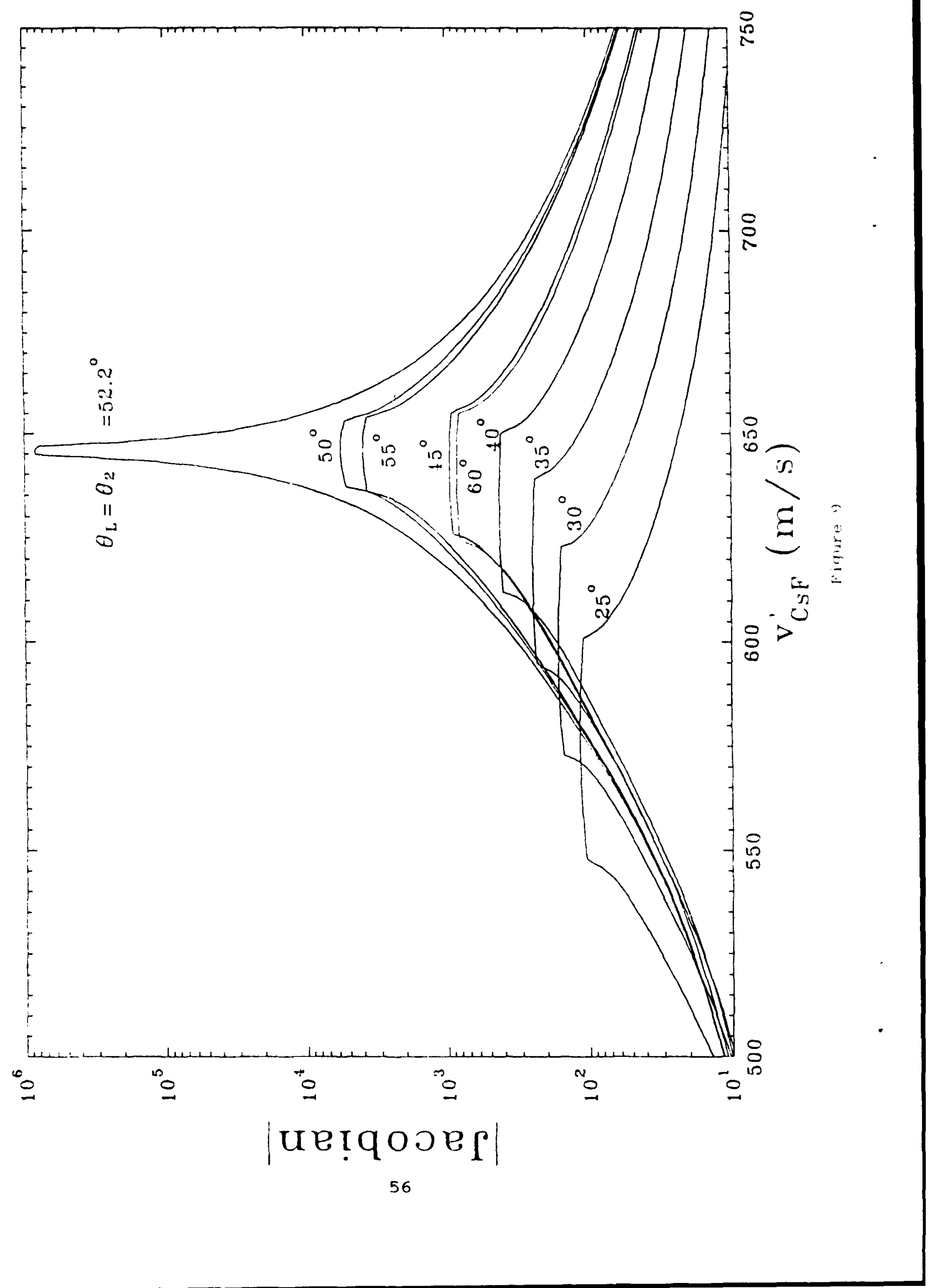




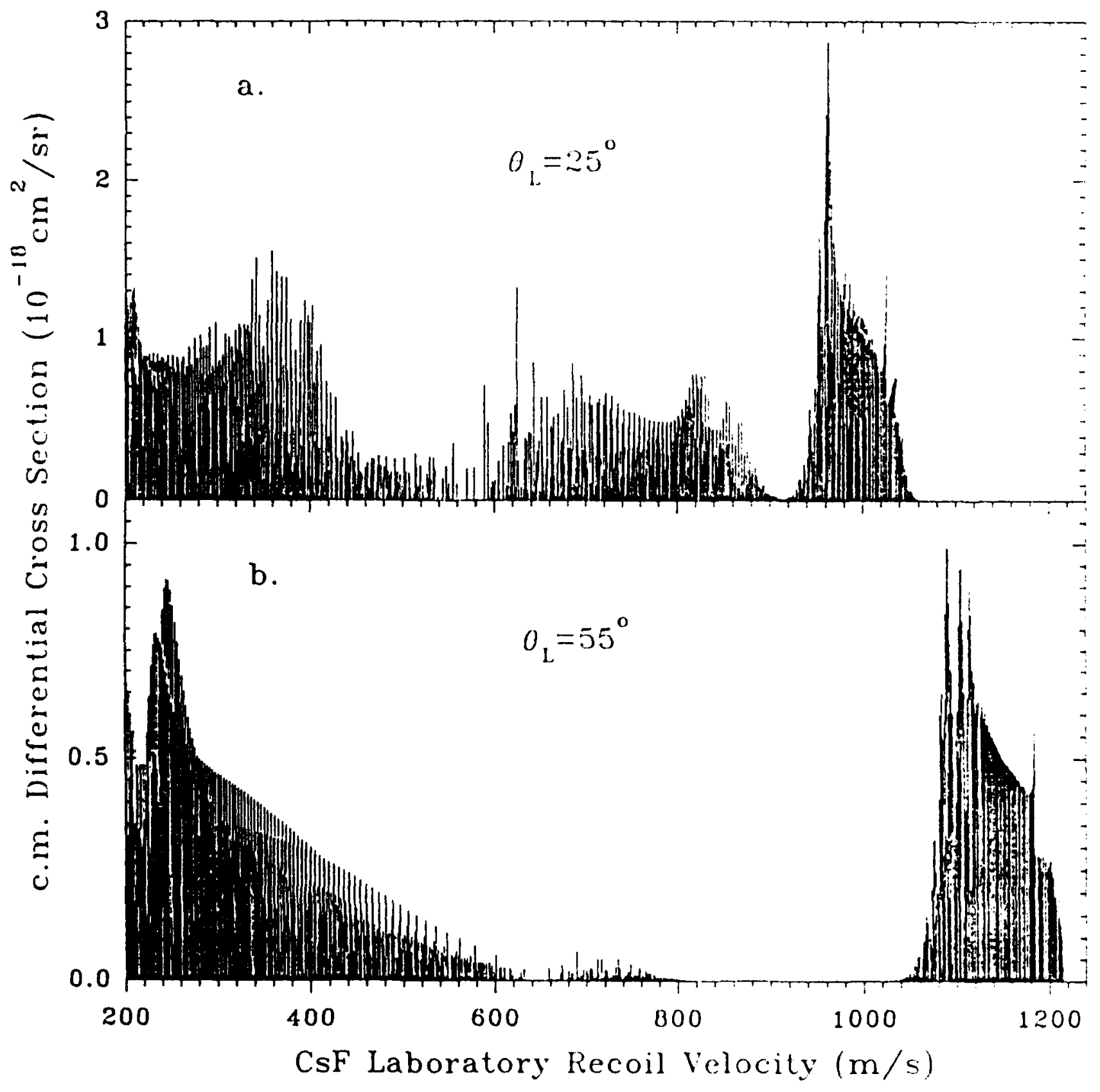



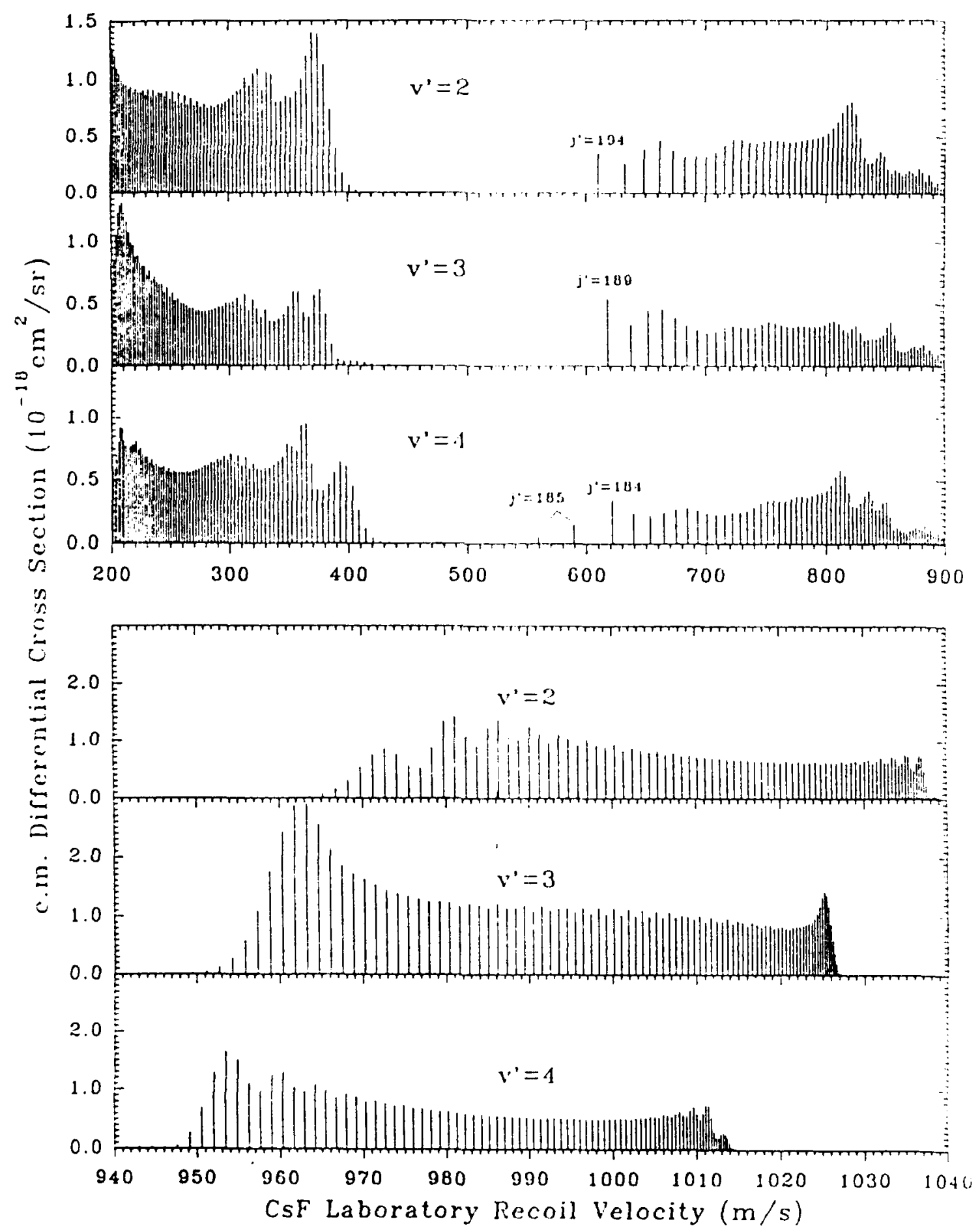


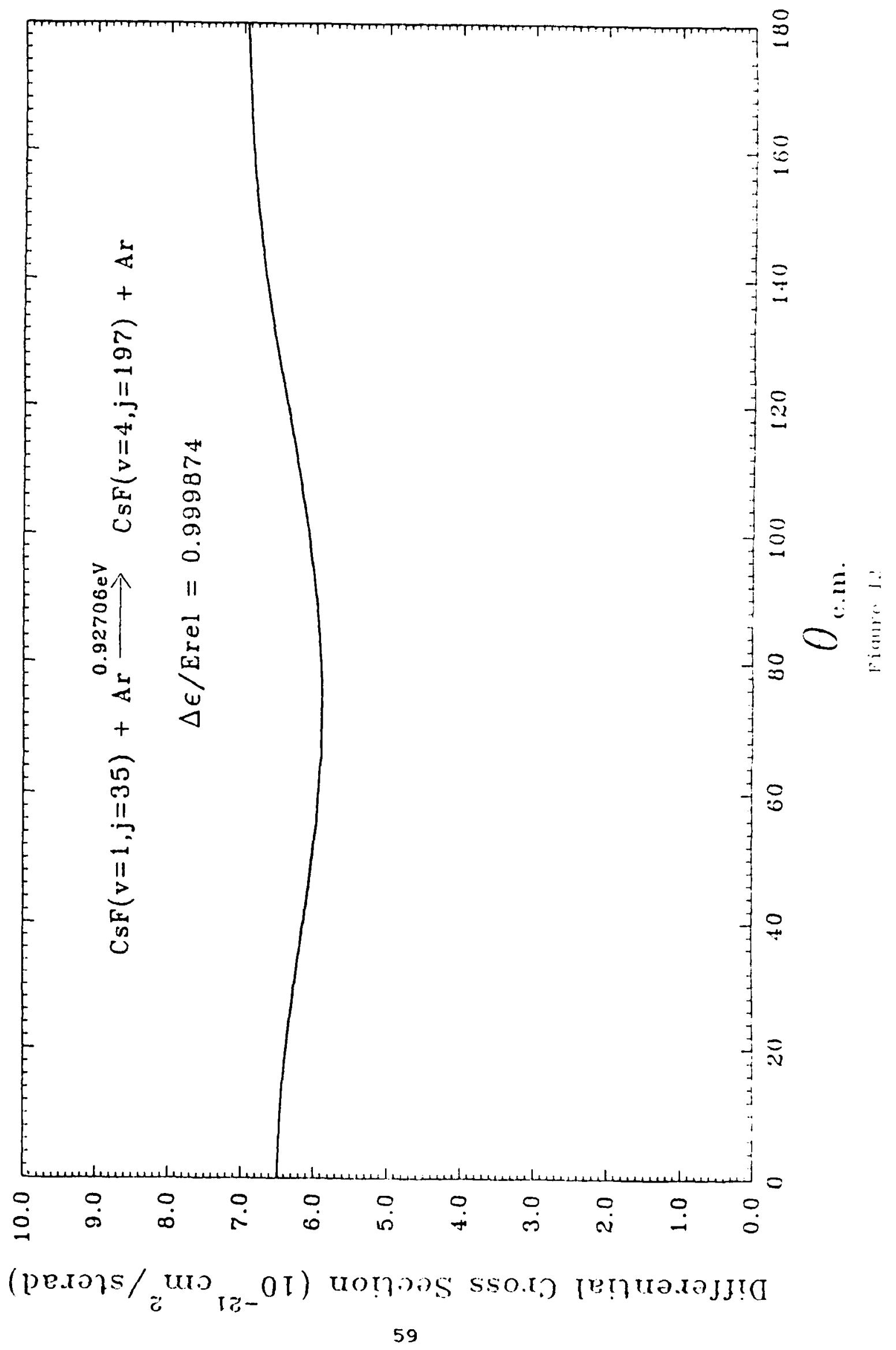




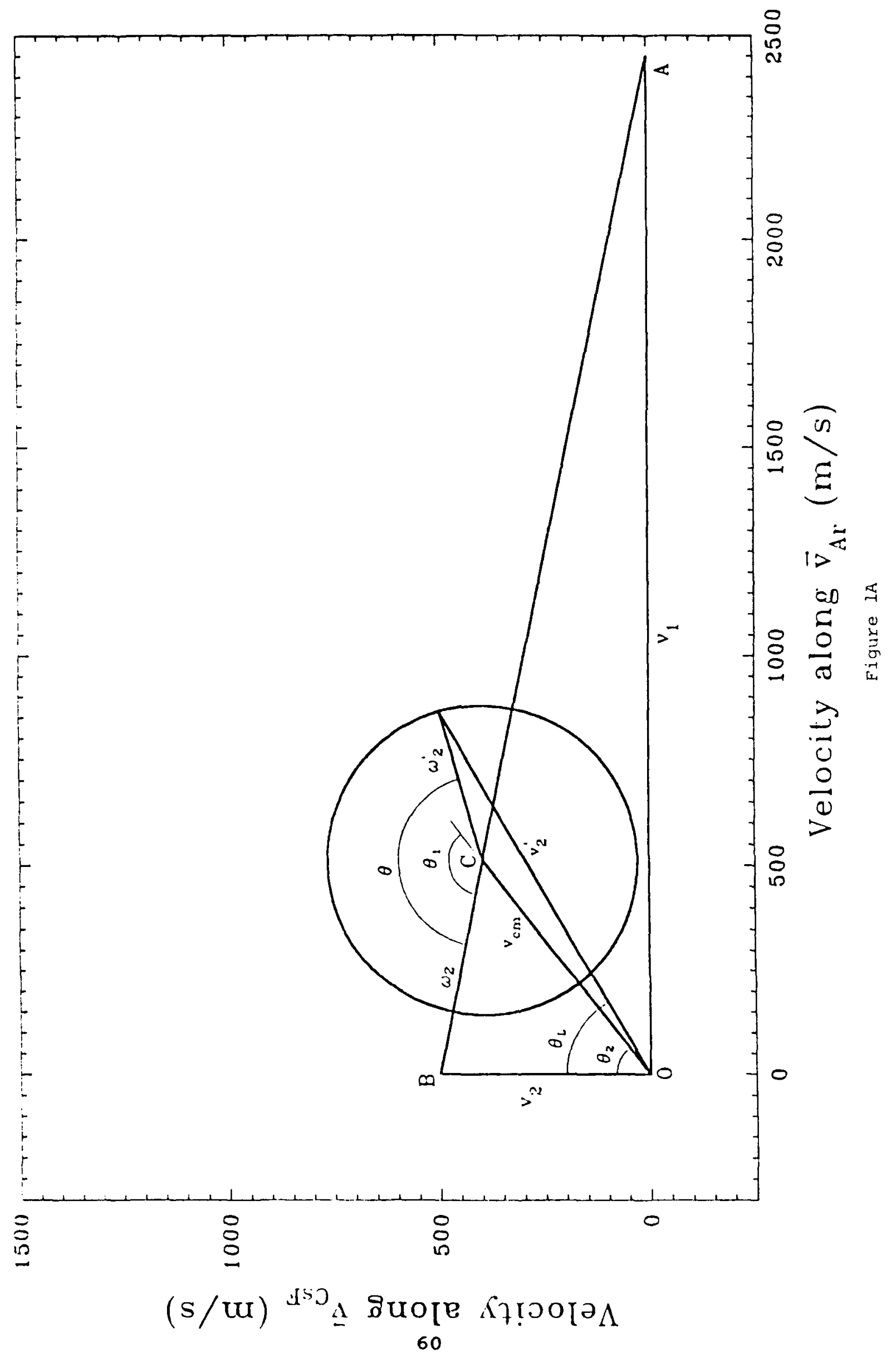




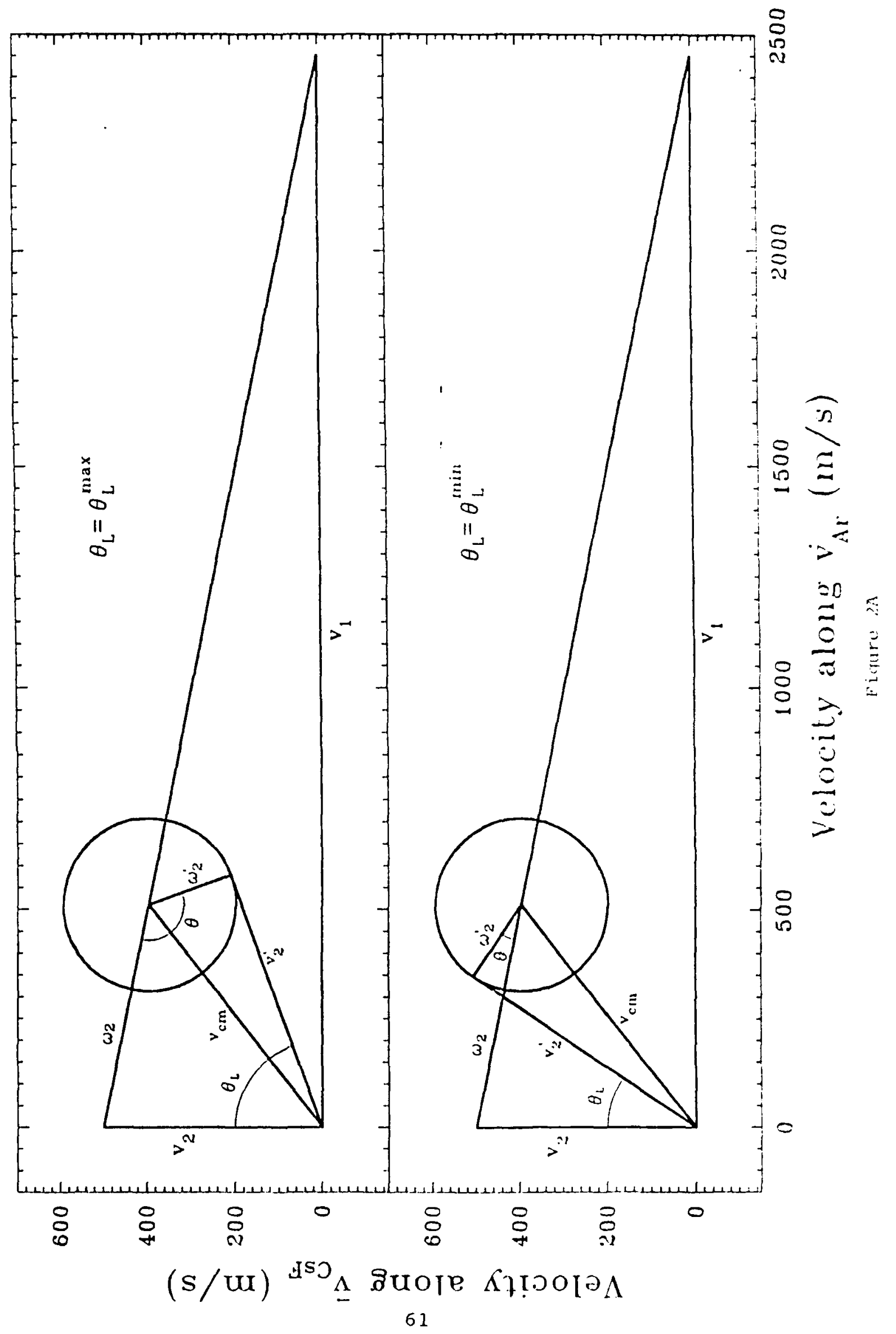




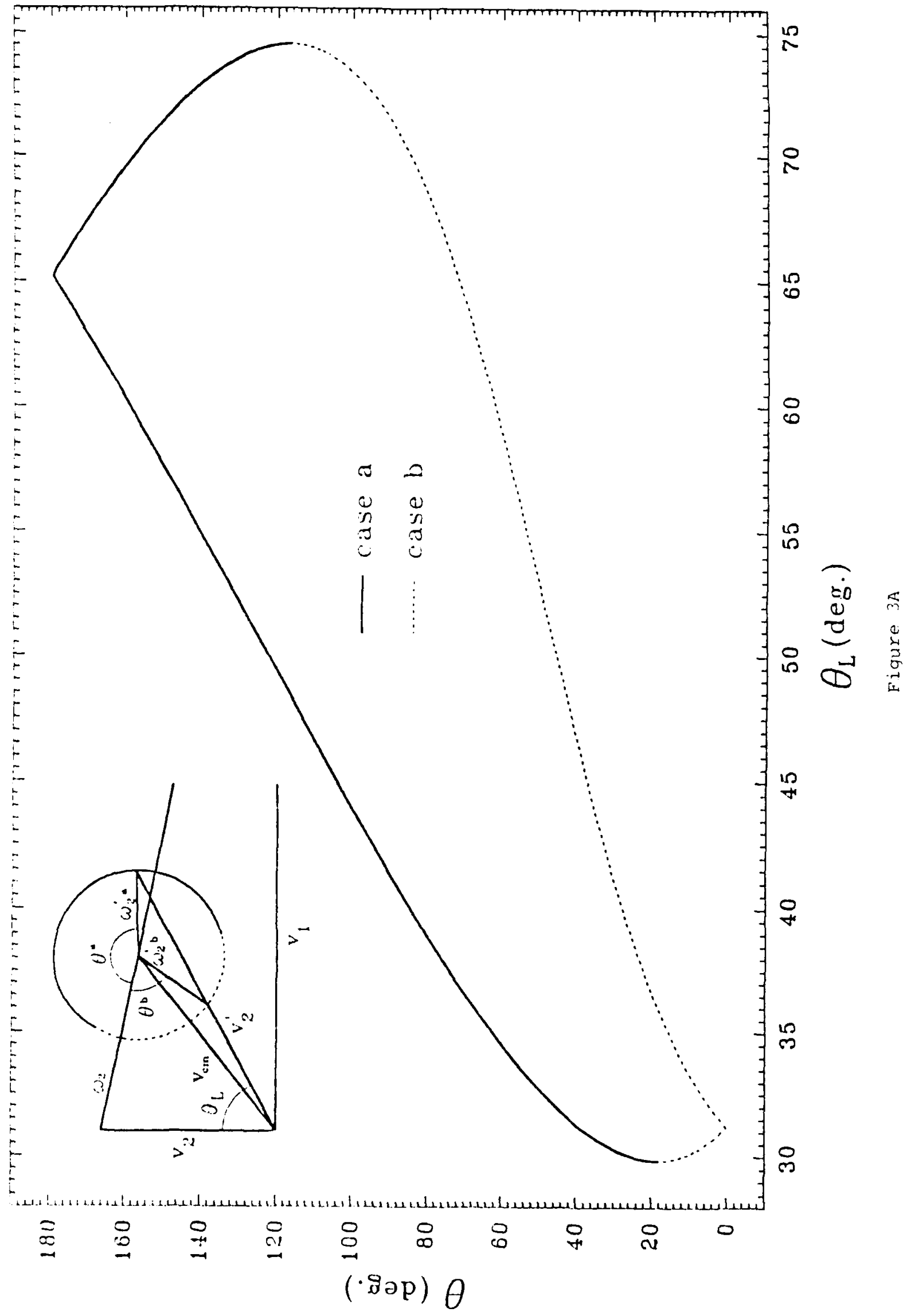




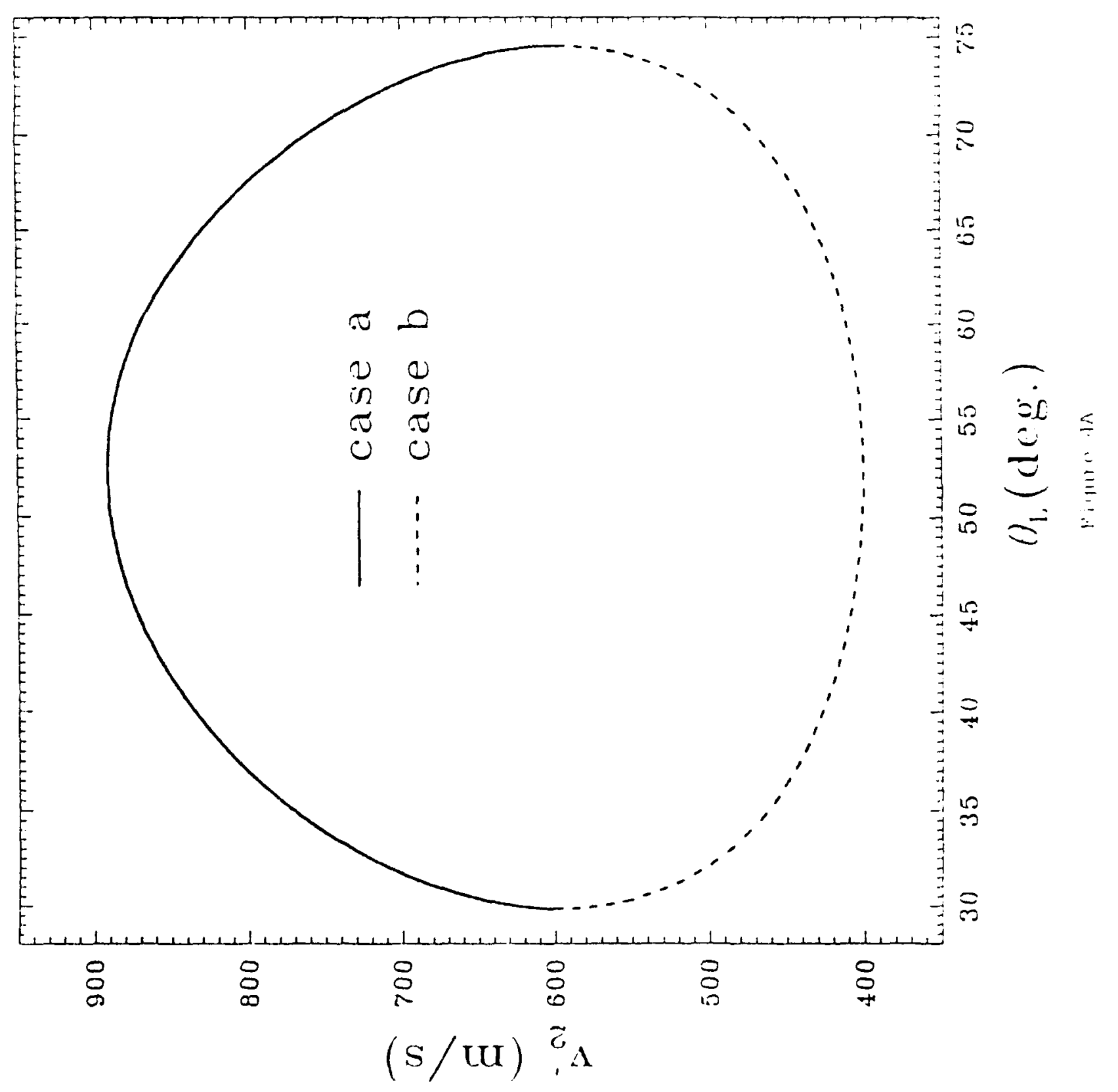




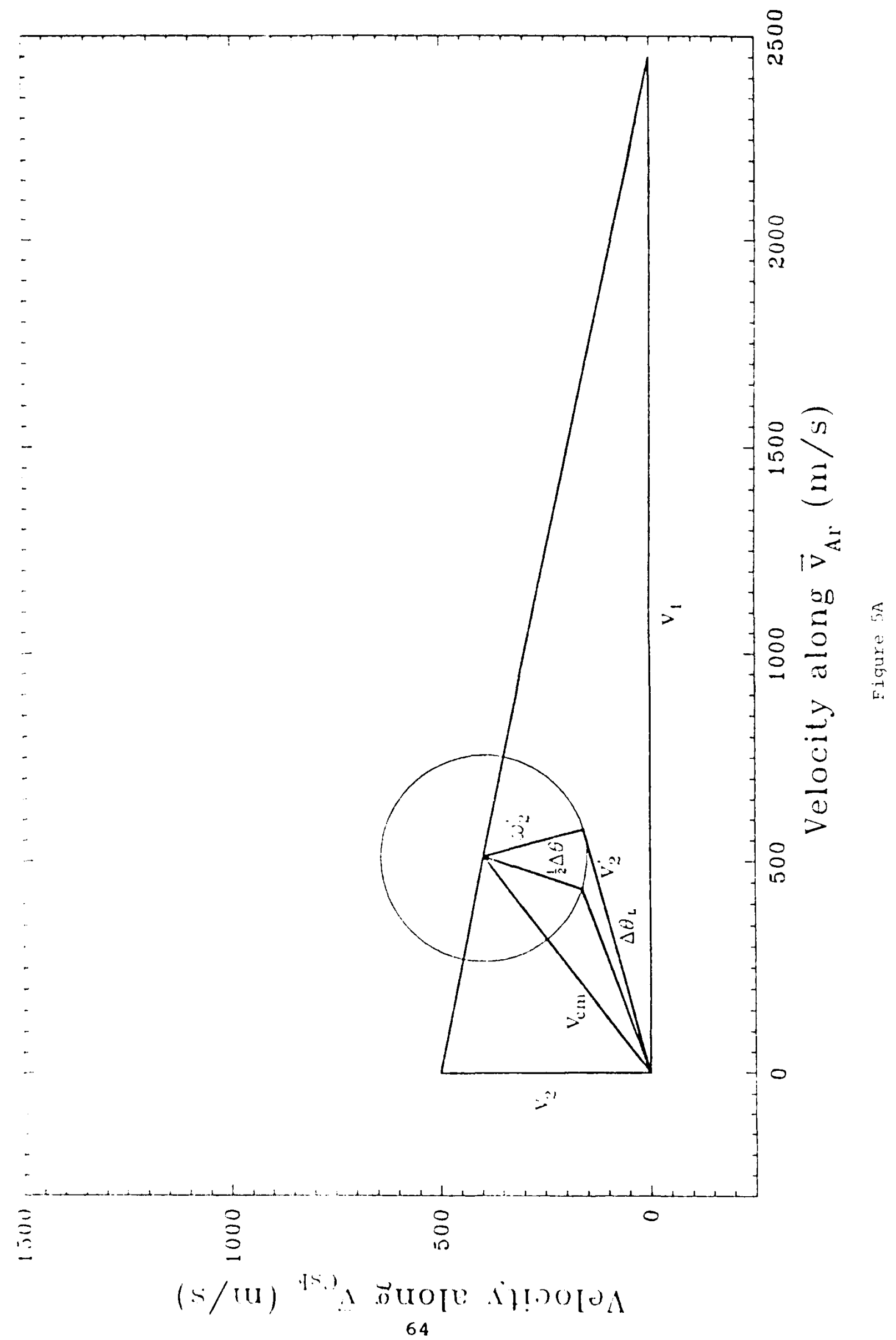


Hoang Dothe

Yap Analytics Inc., Lexington, Massachusetts 02173

$$
\text { Ramesh D. Shama }
$$

Phillips Laboratory, Optical Enviromment Division (GPOS),

Hanscom Air Force Base, Massachuscts $01731-5000$

\section{ABSTRACl}

A formalism is derived to include the effects of the long range attractive part of the interaction potential in the calculation of atom-diatom collision cross sections using the impulse approach (IA). These calculations have, until now, assumed the atom-diatom potential given by a sum of two atom-atom interactions, consequently yielding a poor representation of the long range attractive part. In the Distorted Wave Impulse Approach (DWIA) the long range attractive part, located at the center of mass (c.m.) of the diatom, is a spherically symmetric potential which "distorts" the incoming and outgoing waves. The DWIA formalism is used to calculate differential cross sections for the rotationally inelastic process $\mathrm{Li}^{+}+\mathrm{N}_{2}(v=0, \mathrm{j}=2) \rightarrow \mathrm{Li}^{+}+\mathrm{N}_{2}\left(v^{\prime}=0, j^{\prime}\right)$, as a function of the final rotational level j', at a relative kinetic energy of $4.23 \mathrm{eV}$ and center of mass scattering angles of $49.2^{\circ}$ and $37.1^{\circ}$. It is shown that differential cross sections calculated using the DWIA formalism are in much better agreement with experimentally measured ones than IA differential cross sections using atom-atom interactions expressed by either hard-core, or exponential repulsive, functions.

* Joumal Chem. Phys. (to be publ ished) 


\section{INTRODUCTION}

Recently, an exact formulation of the impulse appioach $(I A)$, or quamimmechanical spectator model, has been developed for atom-diatom collisions [1-3]. Subsequent comparison of computed differential cross sections with experimentally' measured ones has given reasonable results for: (i) the relaxation of highly vibrationally excited $\mathrm{KBr}$ by $\mathrm{Ar}$ [4], (ii) the elastic and inelastic processes at large c.m. scattering angles, and the highly inelastic ballistic processes at all c.m. scattering angles, for the CsF-Ar system [5]. IA calculations [1] have not been able to reproduce the observed differential cross sections for rotational transitions of $\mathrm{N}_{2}$ caused by collisions with $\mathrm{Li}^{+}$in a crossed molecular beam experiment at a relative translational encrgy of $4.23 \mathrm{eV}$ and c.m. scattering angle of about $45^{\circ}$. The calculated distributions of differential cross sections are too narrow and peak at values of the linal rotational quantum number $j$ that are too large. Similar results, for an IA calculation, were obtained earlier by Beard and Micha [6] not only for the $\mathrm{Li}^{+}-\mathrm{N}_{2}$ system but also for the $\mathrm{Li}^{+}-\mathrm{CO}$ system as well. The observed rotational transitions convert only a small fraction of the initial relative translational energy into rotational energy. The situation here is similar to the one encountered earlier [4] in a study of the deactivation of highly excited $\mathrm{KBr}$ by $\mathrm{Ar}$. For nearly elastic scattering at $45^{\circ} \mathrm{cm}$. scattering angle, the IA calculation was not able to describe the experimental results; the calculation, however, agreed well with the experiment when a substantial fraction of nearly $2 \mathrm{eV}$ initial vibrational energy was converted into translational energy. In this paper we seek to improve the calculated distributions of rotationally inelastic differential cross sections.

In the impulse approach, the total atom-diatom interaction potential is described by a sum of two atom-atom interactions. These atum-atom interactions are in all of the calculations on vibrational-rotational excitation of the diatom published thus far, approximated by hard core potentials, i.e., $V(r)=\infty$ for $r \leq d$ and $V(r)=0$ for $r>d, d$ being the suitably chosen hard core radius. To arrive at an understanding of the 
influence of hard core potentials on the calculated differential cross sections, we shall carry out the IA calculation using exponential repulsive atom-atom interactions as well.

While the sum of the two atom-atom interactions appears to be an adequate description of the atom-diatom potential at small distances, this model fails to describe its long range attractive part. To remedy this situation, we propose to add to the sum of pair-interactions a centrally symmetric attractive potential located at the c.m. of the diatom. We shall study the effects of this potential on the differential cross sections of collision-induced rotational transitions involving the conversiun of only a small fraction of the initial relative translational energy into rotational encrgy. The expression for the T matrix in the Distored Wave Impulse Approach (DWIA) will be shown to describe an overall collision process where the incoming particle is first scattered by the central potential located at the c.m. of the diatom (the distorting potential), then scattered by one of its atoms, and, to maintain microscopic reversibility, finally scattered again by the distorting potential. It was pointed out earlier that the impulse calculations [2-5] give reasonable results for large c.m. scattering angles. We will, therefore, also study the difference between the IA and DWIA differential cross sections as a function of c.m. scattering angles.

The paper is organized as follows: in section II, we derive the formal equations of the distorted wave approach; in section III, we derive the necessary mathematical formulae for computing the DWIA scattering amplitude for atom-diatom collisions; in section IV, we present an application of the model to the $\mathrm{Li}^{+}+\mathrm{N}_{2}$ scattering process; finally, in section V, we summarize the results of the calculations. In the Appendix, an expression for the atom-diatom collision amplitude in the impulse approach (l.A) is derived in terms of the incoming and outgoing momenta $\mathbf{p}_{3}$ and $\mathbf{p}_{3}^{\prime}$; this expression is crucial in the formulation of the DWIA scattering amplitude presented in section III. 


\section{THE DISTORTED WAVE FORMALISM}

We follow the notation of ref. I, which we refer to throughout this paper as SBS. The scattering process involves the collision of atom 3 with a molecule consisting of atoms 1 and 2. We make use of the set of Jacobi nomenti, where in a system of 3 particles

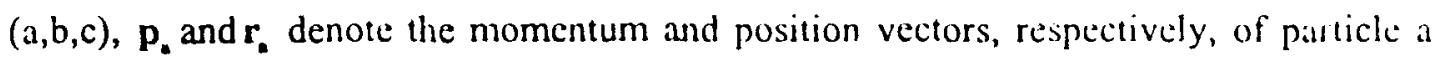
with respect to the center of mass (c.m.) of particles bc; $\mathbf{q}_{\text {. }}$ and $\mathbf{y}_{\text {, }}$, respectively, are the vectors denoting the relative momentum and relative position of particles $b$ and $c ; \mu_{a}, \mu_{b i}$ are the reduced masses of the systems $(a, b c)$ and $(b, c)$, respectively; $V_{n}$ is the potential between particles $b$ and $c$, and the spherically symmetric atom diatom distortion potential located at the c.m. of the molecule is denoted by $V_{0}\left(r_{3}\right)$

The total Hamiltonian of the three particles in the c.m. coordinates of the system is,

$$
H=H_{0}+V_{\text {low }}=H_{0}+V_{3}\left(y_{3}\right)+V
$$

where

$$
\mathrm{H}_{0}=\frac{\mathrm{p}_{3}^{2}}{2 \mu_{3}}+\frac{\mathrm{q}_{3}^{2}}{2 \mu_{12}}
$$

is the kinetic energy operator, $V_{3}\left(y_{3}\right)$ is the intramolecular potential, and $V$, the atomdiatom interaction potential, is the sum of the distortion potential $V_{v}\left(r_{3}\right)$ and impulse potentials $V_{1}\left(y_{1}\right)$ and $V_{2}\left(y_{2}\right)$, i.e.,

$$
V=V_{0}\left(r_{3}\right)+V_{1}=V_{0}\left(r_{3}\right)+V_{1}\left(y_{1}\right)+V_{2}\left(y_{2}\right)
$$

It was mentioned that $V_{0}\left(r_{3}\right)$, the distorting potential, is a spherically symmetric potential located at the $\mathrm{c} . \mathrm{m}$. of the diatom and $\mathrm{V}_{l}$, the impulse potential is the sum of two atom-atom interactions. These restrictions will not be used in the derivation of the formal equations. We will however, for convenience, contincie to refer to $V_{0}$ and $V_{l}$ as 
the distortion and the impulse potential, respectively. The "T operator associated with the total Hamiltonian is

$$
\mathbf{T}(\mathbf{z})=\mathrm{V}+\mathrm{V}\left(\mathbf{z}-\mathrm{H}_{3}-\mathrm{V}\right)^{-1} \mathrm{~V}
$$

where

$$
\mathrm{H}_{3}=\mathrm{H}_{\text {Dimiom }}+\frac{\mathrm{p}_{3}^{2}}{2 \mu_{3}}
$$

is the Hamiltonian at large distances when $\mathrm{V} \rightarrow 0$ and,

$$
H_{\text {Diriom }}=\frac{q_{3}^{2}}{2 \mu_{12}}+V_{3}\left(y_{3}\right)
$$

is the Hamiltonian of the diatom. In eq. (3), $z=E+i \varepsilon$, E bcing the total energy of the atom-diatom system. The eigenfunctions of $\mathrm{H}_{3}$ are given by:

$|\alpha\rangle=\left|u_{\alpha}\right\rangle \otimes\left|\Psi_{\alpha}\right\rangle=\left|\operatorname{vjm} p_{3 \alpha}\right\rangle$

and

$|\beta\rangle=\left|u_{\beta}\right\rangle \otimes\left|\Psi_{\beta}\right\rangle=\left|v^{\prime} \mathrm{j}^{\prime} \mathrm{m}^{\prime} \mathbf{p}_{3 \beta}\right\rangle$,

where $u_{\alpha} \equiv u\left(\mathbf{p}_{3 \alpha}\right), u_{\beta} \equiv u\left(\mathbf{p}_{3 \beta}\right)$ are plane waves, eigenfunctions of $\frac{\mathrm{p}_{3}^{2}}{2 \mu_{3}}$, normalized to delta function, and $\left|\psi_{\alpha}\right\rangle \equiv|v j m\rangle,\left|\psi_{\beta}\right\rangle \equiv\left|v^{\prime} j^{\prime} m^{\prime}\right\rangle$ are vibrational-rotational wave functions, eigenfunctions of $\mathrm{H}_{\text {Dimom }}$, in the initial and final states, respectively. Define the Green's function, or the propagator:

(1) For the unperturbed motion

$$
G_{3}^{+}(z)=\left(z-H_{3}\right)^{-1} \text {, }
$$

(2) For the total Hamiltonian $\mathrm{H}$

$$
G^{+}(z)=\left(z-H_{3}-V_{0}-V_{I}\right)^{-1} \text {, }
$$

(3) For the unperturbed motion and distortion potential

$$
G_{0}^{+}(z)=\left(z-H_{3}-V_{0}\right)^{-1} \text {, }
$$


(4) For the unperturbed motion and impulse potential

$$
G_{i}^{\prime}(z)=\left(z-H_{3}-V_{1}\right)^{\prime}
$$

The T-operator corresponding to the distortion potential is given by

$$
\mathrm{T}_{0}=\mathrm{V}_{0} \Omega_{0} \text {, }
$$

and that corresponding to the impulse potential is

$$
\mathrm{T}_{1}=\mathrm{V}_{1} \Omega_{1} \text {, }
$$

where $\Omega$ is a Moller wave operator defined, for the distorting potential, by

$$
\Omega_{0}=1+G_{0}^{+} V_{0} \text {. }
$$

and for the impulse potential, by

$$
\Omega_{1}=1+G_{1}^{+} V_{1} \text {. }
$$

An exact expression for the scattering amplitude in the distorted wave formalism is given by [7]

$$
\langle\beta|T| \alpha\rangle=\left\langle\beta\left|T_{0}\right| \alpha\right\rangle+\left\langle\chi_{0 \beta}^{T-}\left|V_{1}\right| \chi_{\alpha}^{+}\right\rangle
$$

where $|\alpha\rangle$ and $|\beta\rangle$ are unperturbed wavefunctions for the initial state and the final state, respectively, defined by equations (6a) and (6b). $\chi_{\alpha}^{+}$is the solution of the Lippmann-Schwinger equation,

$\left|\chi_{\alpha}^{+}\right\rangle=\left(1+G^{+} V_{1}\right)\left|\chi_{0 \alpha}^{+}\right\rangle$,

where $\chi_{o c}^{+}$is the outgoing solution of the Lippmann-Schwinger equation with only the distorting potential

$$
\begin{aligned}
\left|\chi_{u \alpha}^{*}\right\rangle & =\Omega_{0}|\alpha\rangle=\left(1+G_{0}^{*} V_{0}\right)|\alpha\rangle \\
& =|\alpha\rangle+G_{3}^{+} V_{0}\left|\chi_{0 \alpha}^{+}\right\rangle \\
& =\left(1+G_{3}^{+} T_{0}\right)|\alpha\rangle,
\end{aligned}
$$


and $\chi_{o \beta}^{r-}$ is the ingoing solution of the Lippmann-Schwinger equation, again with only the distorting potential,

$$
\begin{aligned}
\left\langle\chi_{0 \beta}^{T-}\right| & =\langle\beta| \Omega_{0}=\langle\beta|\left(1+V_{0} G_{0}^{+}\right) \\
& =\langle\beta|+\left\langle\chi_{0 \beta}^{T-}\right| V_{0} G_{3}^{+} \\
& =\langle\beta|\left(1+T_{0} G_{3}^{\prime}\right) .
\end{aligned}
$$

It is seen that $\chi_{\alpha}^{+}$is the solution of the Schröedinger equation with the full Hamiltonian H. An approximate solution to equation (8) is provided by trating $\mathrm{V}_{0}$, the spherically symmetric potential located at the c.m. of the diatom, as a small perturbation. Writing the expression for the Green's function corresponding to the full Hamilionian II as

$$
G^{+}=G_{1}^{+}+G_{1}^{+} V_{0} G^{+}
$$

and keeping only the first term on the right hand side of the equation, i.e., $G^{\prime}=G_{1}^{*}$, we have from equations $(9 \mathrm{a})$ and $(7 \mathrm{~h})$,

$$
\left|\chi_{\alpha}^{+}\right\rangle \approx\left(1+G_{1}^{+} V_{1}\right)\left|\chi_{0 \alpha}^{+}\right\rangle=\Omega_{1}\left|\chi_{0 \alpha}^{+}\right\rangle
$$

Equation (11) has the effect of disentangling the effects of $V_{0}$ and $V_{1}$ and keeping the terms in the lowest order in $V_{0}$. This is the reason that primarily the weak attractive long range portion of the atom-diatom potential will be included in $V_{0}$. From equations (8), (11), and (7f), the scattering amplitude can be written as,

$$
\langle\beta|T| \alpha\rangle \approx\left\langle\beta\left|T_{0}\right| \alpha\right\rangle+\left\langle\chi_{0 \mid 1}^{T-}\left|T_{1}\right| \chi_{i \alpha}^{*}\right\rangle
$$

Substituting equations (9c) and (9b) for $\left\langle\chi_{\text {ipl }}^{\mathrm{r}}\right|$ and $\left|\chi_{0.4}^{+}\right\rangle$into equation (12), we get

$$
\begin{aligned}
\langle\beta|T| \alpha\rangle & =\left\langle\beta\left|T_{0}\right| \alpha\right\rangle+\left\langle\beta\left|\left(1+T_{0} G_{3}\right) T_{1}\left(1+G ; T_{0}\right)\right| \alpha\right\rangle \\
& =\left\langle\beta\left|T_{0}+T_{1}+T_{0} G ; T_{1}+T_{1} G_{3}^{\prime} T_{0}+T_{0} G_{;}^{\prime} T_{1} G_{;}^{\prime} T_{0}\right| \alpha\right\rangle
\end{aligned}
$$


We can see here that the operator on the right side of equation (13) is just the first few terms in the expansion of the three-body T-matrix in a multiple collision series $[8,9]$. The complete formal equivalence between the distorted wave formalism and the multiple collision series approach can be established by using the exact expression for $\mathrm{G}^{+}$(eq. 10). It is worth mentioning again that all of the above relations are completely general. They were obtained without any assumption about the forms of the interaction potentials. Equations (12) and (13) give the scattering amplitude in the Distorted Wave formalism, subject to the approximation $G^{*} \approx G_{1}^{+}$in eq. (10), i.e., in the lowest order in $V_{0}$.

The equations derived thus far do not make use of the fact that $V_{0}$ is spherically symmetric. For rotationally inelastic transitions, we can ignore the effect of $T_{0}$ alone, since $V_{0}$ contributes only to rotationally elastic processes. We shall therefore concentrate only on the second term in eq. (12).

\section{MATHEMATICAL FORMULAE}

We first write the potential $V_{I}$ as a sum of two atom-atom interactions,

$$
V_{1}\left(r_{3}, y_{3}, \alpha\right)=V_{1}\left(y_{1}\right)+V_{2}\left(y_{2}\right)
$$

where $r_{3}$ is the distance between the incident atom 3 and the c.m. of the diatom $1-2, \alpha$ is the angle between $r_{3}$ and $y_{3}$, and

$y_{1}\left(y_{2}\right)=\left\{r_{3}^{2}+\left[c_{1}\left(c_{2}\right) y_{3}\right]^{2} \mp 2 r_{3} y_{3} c_{1}\left(c_{2}\right) \cos \alpha\right\}^{\frac{1}{2}}$,

where

$$
c_{1}\left(c_{2}\right) \equiv m_{2}\left(m_{1}\right) /\left(m_{1}+m_{2}\right)
$$

is the appropriate mass ratio. The total Hamiltonian can then be written as,

$$
H=H_{3}+V_{1}+V_{0}\left(r_{3}\right)=H_{3}+V_{1}\left(y_{1}\right)+V_{2}\left(y_{2}\right)+V_{0}\left(r_{3}\right)
$$

and the three-body $T_{I}$ matrix expanded in a multiple-collision series, 


$$
\begin{aligned}
T_{1}= & T^{(1)}+T^{(2)}+T^{(1)} G_{3}^{\prime} T^{(2)}+T^{(2)} G_{3}^{\prime} T^{(1)} \\
& +T^{(1)} G_{3}^{+} T^{(2)} G_{3}^{+} T^{(1)}+T^{(2)} G_{3}^{\prime} T^{(1)} G_{3}^{\prime} T^{(2)}+\cdots
\end{aligned}
$$

where the three-body operators $T^{(0)}$ denote the collision of the incident atom with atom $s^{\prime}$ of the diatom, $s$ being the spectator atom. $T^{(0)}$ is defined by,

$$
\begin{aligned}
& T^{(0)}=V_{.} \Omega_{\bullet}, \\
& \Omega_{.}=1+\left(z-H_{3}-V_{.}\right)^{-1} V_{.},
\end{aligned}
$$

$s=1,2$. Substituting equation (18) into the second term on the right hand side of equation (12) and keeping only the first two terms, i.e., keeping only the single collision terms in the multiple collision expansion of the three-body T-matrix, we obtain the matrix elements

$$
\sum_{\eta, \xi}\left\langle\beta\left|1+\mathrm{T}_{0} \mathrm{G}_{3}^{+}\right| \xi\right\rangle\left\langle\xi\left|\mathrm{T}^{(0)}\right| \eta\right\rangle\left\langle\eta\left|1+\mathrm{G}_{3}^{+} \mathrm{T}_{0}\right| \alpha\right\rangle
$$

where $|\alpha\rangle \equiv\left|v j m p_{3 \alpha}\right\rangle$ and $|\beta\rangle \equiv\left|v^{\prime} j^{\prime} m^{\prime} p_{3 \mu}\right\rangle, s=1,2$. We have used the completenesi relations $\sum_{\eta}|\eta\rangle\langle\eta|=1$ and $\sum_{\xi}|\xi\rangle\langle\xi|=1$, the summation denoting sum over the discrete indices and integration over the continuous ones. In eq. (17), we assumed that $V_{0}$ is a spherically symmetric potential. Now we further restrict $V_{0}$ by requiring it to be independent of the vibrational coordinate. Since the dependence of the atom-diatom potential on the vibrational coordinate is usually not known, this is not the limiting factor in determining the accuracy of the calculation. $T_{0}$ can thus give rise to only rotationally and vibrationally elastic transitions. The sums in equation (21) are then non vanishing only for $|\eta\rangle=\left|v j m p_{3}\right\rangle$ and $|\xi\rangle=\left|v^{\prime} j^{\prime} m^{\prime} p_{3}^{\prime}\right\rangle$. The integration is therefore only over $p_{3}$ and $p_{3}^{\prime}$. 
The first and third matrix elements in the integrand can then be written as,

$$
\begin{aligned}
& \left\langle u\left(\mathbf{p}_{3 \beta}\right)\left|1+\mathrm{T}_{0} \mathrm{G}_{3}^{+}\right| u\left(\mathbf{p}_{3}^{\prime}\right)\right\rangle \\
& =\delta\left(\mathbf{p}_{3}^{\prime}-\mathbf{p}_{3 \beta}\right)+\frac{2 \mu_{3}}{\hbar^{2}} \frac{\left\langle u^{T-}\left(\mathbf{p}_{3 \beta}\right)\left|V_{0}\right| u\left(\mathbf{p}_{3}^{\prime}\right)\right\rangle}{\left(p_{3 \beta}^{2}-p_{3}^{\prime 2}\right)+i \varepsilon}
\end{aligned}
$$

and

$$
\begin{aligned}
& \left\langle u\left(\mathbf{p}_{3}\right)\left|1+\mathrm{G}_{3}^{+} \mathrm{T}_{\mathbf{0}}\right| u\left(\mathbf{p}_{3 \alpha}\right)\right\rangle \\
& =\delta\left(\mathbf{p}_{3}-\mathbf{p}_{3 \alpha}\right)+\frac{2 \mu_{3}}{h^{2}} \frac{\left\langle u\left(\mathbf{p}_{3}\right)\left|\mathrm{V}_{0}\right| u^{+}\left(\mathbf{p}_{3 \alpha}\right)\right\rangle}{\left(\mathbf{p}_{3 \alpha}^{2}-\mathrm{p}_{3}^{2}\right)+\mathrm{i \varepsilon}},
\end{aligned}
$$

where we have used eqs. $(7 \mathrm{~g}),(9 \mathrm{~b})$ and $(9 \mathrm{c})$, and the limit $\varepsilon \rightarrow 0^{+}$is implied. The wave functions for the relative translational motion are defined as follows; $u(\mathbf{k})$ is the plane wave for free translational motion

$$
\begin{aligned}
\left\langle\mathbf{r}_{3} \mid u(\mathbf{k})\right\rangle & =(2 \pi)^{-3 / 2} \exp \left(\mathrm{ik} \cdot \mathbf{r}_{3}\right) \\
& =\left(\frac{2}{\pi}\right)^{1 / 2} \sum_{\mathbf{k}} \mathrm{i}^{\mathrm{k}} \mathrm{j}_{\mathbf{k}}\left(\mathrm{kr} \mathrm{r}_{3}\right) \sum_{\gamma} \mathrm{Y}_{\mathrm{kr \gamma}}(\hat{\mathbf{k}}) \mathrm{Y}_{\mathrm{r \gamma}}^{*}\left(\hat{\mathbf{r}}_{3}\right),
\end{aligned}
$$

$u^{T-}\left(k^{\prime}\right)$ is the solution for the translational motion which asymptotically goes into a plane wave and an ingoing spherical wave

$$
\left\langle u^{T-}\left(k^{\prime}\right) \mid \mathbf{r}_{3}\right\rangle=\left(\frac{2}{\pi}\right)^{1 / 2} \sum_{k^{\prime}}(-i)^{\kappa^{\prime}} \omega_{\kappa^{\prime}}^{*}\left(k^{\prime} r_{3} ; \varepsilon^{-}\right) \sum_{\gamma^{\prime}=-k^{\prime}}^{k^{\prime}} Y_{k^{\prime} \gamma^{\prime}}^{*}\left(\hat{k}^{\prime}\right) Y_{k^{\prime} \gamma^{\prime}}\left(\hat{\mathbf{r}}_{3}\right)
$$

and $u^{+}\left(k^{\prime}\right)$ is the solution for the translational motion which asymptotically goes into a plane wave and an outgoing spherical wave

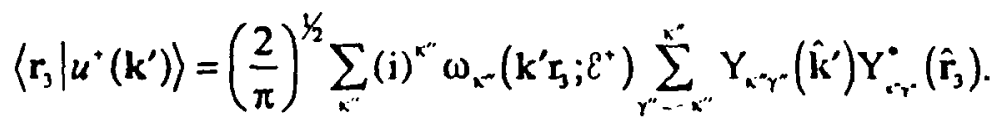

The radial wavefunctions $\omega_{\ell}\left(k^{\prime} r_{3} ; \mathcal{E}^{ \pm}\right)$are solutions of the Schröedinger equation 


$$
\left[\varepsilon+\frac{\hbar^{2}}{2 \mu_{3}}\left\{\frac{\partial^{2}}{\partial r_{3}^{2}}+\frac{2}{r_{3}} \frac{\partial}{\partial r_{3}}-\frac{\ell(\ell+1)}{r_{3}^{2}}\right\}-v_{0}\left(r_{3}\right)\right] \omega_{\ell}\left(k^{\prime} r_{3} ; \ell^{\prime}\right)=0,
$$

where

$$
\varepsilon=\frac{\hbar^{2} k^{\prime 2}}{2 \mu_{3}}
$$

and $\omega_{1}\left(\mathrm{k}^{\prime} \mathrm{r}_{3} ; \varepsilon^{*}\right)$ satisfy the outgoing and incoming asymptotic boundary conditions

$$
\omega_{\ell}\left(k^{\prime} r_{3} ; \varepsilon^{ \pm}\right)=\frac{\exp \left( \pm i \delta_{0 \ell}\right)}{k^{\prime} r_{3}} \sin \left(k^{\prime} r_{3}-\ell \frac{\pi}{2}+\delta_{0 \ell}\right)
$$

$\delta_{0 \text { e }}$ being the real phase shift. Substituting equations (24) and (25a) into equation (22), the right hand side of equation (22) becomes,

$$
\begin{aligned}
& \sum_{\tau_{1}}\left[\frac{\delta\left(p_{3}^{\prime}-p_{3 \beta}\right)}{p_{3 \beta}^{2}}+\frac{2 \mu_{3}}{\hbar^{2}} \frac{t_{0 \ell_{i}}\left(p_{3}^{\prime}, p_{3 \beta}\right)}{p_{3 \beta}^{2}-p_{3}^{\prime 2}+i \varepsilon}\right] \sum_{m i} Y_{l_{i}^{\prime} m_{i}^{\prime}}^{*}\left(\hat{p}_{3 \beta}\right) Y_{\ell_{i}^{\prime} m_{i}^{\prime}}\left(\hat{p}_{3}^{\prime}\right) \equiv \\
& \sum_{\tau_{i}} S_{r_{1}}\left(p_{3}^{\prime}, p_{3 \beta}\right) \sum_{m_{i}^{\prime}} Y_{l_{i}^{\prime} m_{i}^{\prime}}^{*}\left(\hat{p}_{3 \beta}\right) Y_{l_{1} m_{i}^{\prime}}\left(\hat{p}_{3}^{\prime}\right),
\end{aligned}
$$

where,

$$
\begin{aligned}
t_{0 \ell}\left(k, k^{\prime}\right) & \equiv \frac{2}{\pi} \int_{0}^{-} \omega_{\ell}^{\prime}\left(k^{\prime} r_{3} ; \varepsilon^{-}\right) V_{0}\left(r_{3}\right) j_{\ell}\left(k r_{3}\right) r_{3}^{2} d r_{3} \\
& =\frac{2}{\pi} \int_{0}^{-} j_{\ell}\left(k r_{3}\right) V_{0}\left(r_{3}\right) \omega_{\ell}\left(k^{\prime} r_{3} ; \varepsilon^{+}\right) r_{3}^{2} d r_{3} .
\end{aligned}
$$

In a similar way, we can rewrite the right hand side of eq. (23) as,

$$
\begin{aligned}
& \sum_{i_{1}}\left[\frac{\delta\left(p_{3}-p_{3 \alpha}\right)}{p_{3 a}^{2}}+\frac{2 \mu_{3}}{\hbar^{2}} \frac{t_{0 \ell_{1}}\left(p_{3}, p_{3 a}\right)}{p_{3 \alpha}^{2}-p_{3}^{2}+i \varepsilon}\right] \sum_{m_{1}} Y_{\ell_{1} m_{1}}\left(\hat{p}_{3 \alpha}\right) Y_{i_{1} m_{1}}^{*}\left(\hat{p}_{3}\right) \equiv \\
& \sum_{i_{1}} S_{l_{1}}\left(p_{3}, p_{3 \alpha}\right) \sum_{i i_{1}} Y_{l_{1} m_{1}}\left(\hat{p}_{3 \alpha}\right) Y_{l_{1} m_{1}}^{*}\left(\hat{p}_{3}\right) .
\end{aligned}
$$


The second matrix element in the integrand of eq. (21) has been evaluated in SBS using the IA. The expression given in that paper was in terms of the momentum transferred $\mathbf{q}=\mathbf{p}_{\mathbf{3}}^{\prime}-\mathbf{p}_{3}$, which is uniquely defined in the single collision case. The present situation defines the momentum transferred vector as $\mathbf{q}=\mathbf{p}_{3 !}-\mathbf{p}_{\mathbf{3}}$. This momentum transfer however occurs in three steps and the DWIA model calls for an integration over $\mathbf{p}_{3}$ and $\mathbf{p}_{3}^{\prime}$, as indicated in eq. (21), muking the momentum transferred in the middle step no longer unique. To carry out this integration the impulse transition amplitude in the equation must be expressed in terms of $\mathbf{p}_{3}$ and $\mathbf{p}_{3}^{\prime}$, rather than the momentum transferred. The needed results are derived in the Appendix, providing

$$
\begin{aligned}
& \left\langle v^{\prime} j^{\prime} m^{\prime} \mathbf{p}_{3}^{\prime}\left|T^{(s)}\right| v j m p_{3}\right\rangle= \\
& \sum_{J M_{1}}\left[\frac{[j][J]}{\left[j^{\prime}\right]}\right]^{1 / 2} C\left(j J j^{\prime} ; m M_{3} m^{\prime}\right) \sum_{\lambda \mu \lambda^{\prime} \mu^{\prime}} A_{j \mu^{\prime}}^{(0)} \cdot\left(v^{\prime} j^{\prime} p_{3}^{\prime} ; v j p_{3}\right) C\left(\lambda \lambda^{\prime} J ; \mu \mu^{\prime} M_{3}\right) Y_{\lambda \mu}^{*}\left(\hat{p}_{3}\right) Y_{\lambda \psi^{\prime}}^{*}\left(\hat{p}_{3}^{\prime}\right),
\end{aligned}
$$

where $[j] \equiv(2 j+1), \quad C^{\prime}$ are the Clebsch-Gordan coefficients, and the quantities $A_{j \lambda^{\prime}}^{(\bullet)}\left(v^{\prime} j^{\prime} p_{3}^{\prime} ; v_{j p}\right)$ are defined by either eq. (A22) or eq. (A27). Equation (29) is the equivalent of equation (49) in SBS, which gives the T matrix in terms of the momentum transferred $\mathbf{q}=\mathbf{p}_{3}^{\prime}-\mathbf{p}_{3}$. Of course, both formulations give the same numerical results.

We can now take the product of the right sides of eqs. (26), (28) and (29), and integrate over $\mathbf{p}_{3}$ and $\mathbf{p}_{3}^{\prime}$, as indicated in eq. (21). Defining the quantity

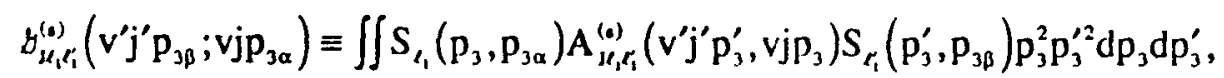

the rotationally inelastic DWIA scattering amplitude, from eq. (13), is then given by

$$
\sum_{\eta, \xi}\left\langle\beta\left|1+T_{0} G_{3}^{+}\right| \xi\right\rangle\left\langle\xi\left|T_{1}\right| \eta\right\rangle\left\langle\eta\left|1+G_{3}^{+} T_{0}\right| \alpha\right\rangle=
$$




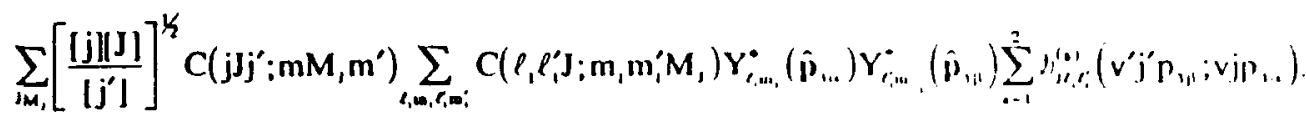

The differential cross section for scattering at an angle $\theta$, in the center of mass frame, for a vibro-rotational transition is given by [9]:

$\frac{d \sigma}{d \Omega}\left(v j p_{3 \alpha} \rightarrow v^{\prime} j^{\prime} p_{3 \beta} ; \theta\right)=\left(\frac{2 \pi}{h}\right)^{4} \mu_{3}^{2} \frac{p_{3 \beta}}{p_{3 \alpha}(2 j+1)} \sum_{m, n^{\prime}}|\langle\beta|T| \alpha\rangle|^{2}$

We can then sum the absolute square of the collision amplitude given by eq. (31) over $\mathrm{m}$ and $m^{\prime}$, or $m$ and $M_{3}=m^{\prime}-m$. Using the identity

$\sum_{j} C\left(j j^{\prime} ; m_{3}\right) C\left(j^{j} j^{\prime} ; m M_{1}\right)=\left[\frac{\left[j^{\prime}\right]}{[j]}\right] \delta_{j,}$

where the second factor on the left hand side comes from the complex conjugate of eq. (31), and taking the $z$ axis of the coordinate system along the direction of the incident momentum $\mathbf{p}_{\mathbf{3} \alpha}$, we finally obtain

$\frac{d \sigma}{d \Omega}\left(v j p_{3 \alpha} \rightarrow v^{\prime} j^{\prime} p_{3 \beta} ; \theta\right)=\left[\frac{2 \pi}{\hbar}\right]^{4} \mu_{3}^{2} \frac{p_{3 \beta}}{p_{3 \alpha}} \sum_{I=j_{1}}^{i+j^{\prime}} \sum_{M_{1}}\left|G_{1 M_{1}}\right|^{2}$,

where

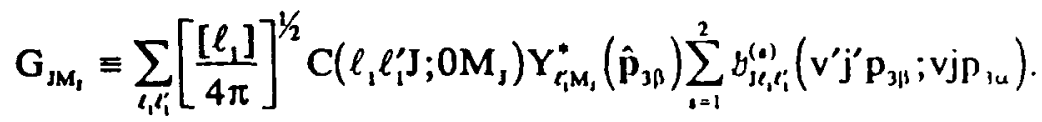

In the next section we shall apply the DWIA model to study the rotationally inelastic scattering of a $\mathrm{N}_{2}$ molecule by a $\mathrm{Li}^{+}$ion, a system that has been extensively studied both experimentally $[10,11]$ and theoretically $[1,6,9,12]$. 


\section{APPLICATION TO THE Li ${ }^{+}+\mathrm{N}_{2}$ SYSTLM}

\section{CHOICE OF POTENTIALS:}

SCF calculations of the $\mathrm{Li}^{+}-\mathrm{N}_{2}$ potential hypersurface have been performed by Staemmler [13]. The calculated energies as a function of the distance between the ion and the center of mass of the molecule are shown in fig. 1 for the collinear $\left(\alpha=0^{\circ}\right)$ and the $C_{2 v}\left(\alpha=90^{\circ}\right)$ configurations. The molecular internuclear separation was kept fixed at the equilibrium distance. Fig. 1 also shows that the repulsive part of the potential above $\mathrm{leV}$ can be approximated using simple exponentials [9]:

$V_{\text {repuline }}=V_{1}\left(y_{1}\right)+V_{2}\left(y_{2}\right)$

where,

$V_{i}\left(y_{i}\right)=B \exp \left(-\beta y_{i}\right)$,

$\mathrm{i}=1,2$

with $\mathrm{B}=1.55 \times 10^{3} \mathrm{eV}$ and $\beta=4.65 \AA^{-1}$.

For large relative kinetic energies of the ion and molecule, it is also possible to replace the above exponential forms by simpler hard core potentials,

$$
\begin{aligned}
V_{i}\left(y_{i}\right) & =\infty & & y_{i} \leq R_{c} \\
& =0 & & y_{i}>R_{c} .
\end{aligned}
$$

The hard core potential with radius $R_{c}$ equal to $1.4 \AA$ is also shown in figure 1 . Similar hard core radii have been used in the previous studies [1,9] of $\mathrm{Li}^{+}-\mathrm{N}_{2}$ collisions. $R_{c}=1.4 \AA$ corresponds to about $4 \mathrm{eV}$ potential energy using the exponential form of the potential function given in equation (37).

It was pointed out earlier that the impulse potential, sum of either the two hard core or the two exponential repulsive functions, cannot, of course, provide a representation of the long range attractive portion of the atom-diatom pctential. The 
DWIA model secks to remedy this situation by adding to the impulse potential at spherically symmetric potential located at the c.m. of the diatom $v_{v}\left(r_{3}\right)$, i.e.,

$V=V_{1}\left(y_{1}\right)+V_{2}\left(y_{2}\right)+V_{0}\left(r_{3}\right)$

and allow $\mathrm{V}_{0}\left(\mathrm{r}_{3}\right)$ to "distort" the incoming and outgoing waves. It should be emphasized that in this model, $\mathrm{V}_{0}\left(\mathrm{r}_{3}\right)$ merely denotes a long range potential chosen in conjunction with the impulse potential to improve the fit to the total atom-diatom potential $V$, which may be determined experimentally or by ab initio calculations. $V_{v}\left(r_{3}\right)$ is not related to $U_{0}\left(r_{3}\right)$ from the standard expansion $V\left(r_{3}, \alpha\right)=\sum_{10}^{\infty} U_{1}\left(r_{3}\right) P_{1}(\cos \alpha)$. We also realize that in choosing a spherically symmetric function to represent the attractive potential, we have assumed that long range anisotropic potentials, e.g., the ion-dipole and ionquadrupole interactions, can be neglected at the higher relative kinetic energies that we are dealing with in this paper. It is however useful to point out that the long range anisotropic potentials can be represented by adding a long range spherically symmetric portion to the impulse potentials. For example, the long range dipole potential is obtained by letting the impulse potentials approach the coulomb interaction at large distances.

The spherically symmetric potential $\mathrm{U}_{0}\left(\mathrm{r}_{3}\right)$ for $\mathrm{Li}^{+}-\mathrm{N}_{2}$ has been determined from the measured total cross section by Gislason, Polak-Dingels and Rajan (GPR) [14]. $V_{0}\left(r_{3}\right)$ is determined from GPR potential as follows: With the hard core radius for the atom-atom potential equal to $1.4 \AA$ and the internuclear distance of the $\mathrm{N}_{2}$ molecule equal to its equilibrium value of $1.1 \AA$, the $\mathrm{Li}^{+}$ion encounters a wall of infinite potential at a distance $r_{3} \approx 1.95 \AA$ in the collinear geometry. We therefore chose as $V_{0}\left(r_{3}\right)$ the GPR potential for the domain $1.95 A \leq r_{3} \leq 5.38 \AA$, the strongly repulsive part of the GPR potential in the inside region $r_{3} \leq 1.95 \AA$ being already accounted for by the hard 
core atom -atom potentials. The value of the GPR potential at $1.95 \AA$, approximately 0.4 $e V$, is therefore extended to $0<r_{3} \leq 1.95 \AA$, resulting in the central potential $V_{0}\left(r_{3}\right)$ shown in fig. (2a).

Figs. (2b) and (2c) show the total potential, given by eq.(39), for the collinear and $\mathrm{C}_{2 \mathrm{v}}$ geometries, respectively. It can be seen by comparing to fig. 1 that the addition of the central potential $V_{0}\left(r_{3}\right)$ has improved the fit to the points obtained by the ab initio calculation in the lower energy region. The small (absolute) energy values of $V_{0}\left(r_{3}\right)$, compared to the values of other potential energies justifies treating $V_{0}\left(r_{3}\right)$ as a perturbation.

\section{2. calculations}

We have calculated c.m. differential cross sections for the rotationally inelastic process $\mathrm{Li}^{+}+\mathrm{N}_{2}(v=0, j=2) \rightarrow \mathrm{Li}^{+}+\mathrm{N}_{2}\left(v=0, \mathrm{j}^{\prime}\right)$, using:

(i) the IA model and hard core atom-atom impulse potentials of equations (36) and (38).

(ii) the IA model and exponential repulsive atom-atom impulse potentials of equations (36) and (37).

(iii) the DWIA model and hard core atom-atom impulse potentials of equations (36) and (38) plus the distorting potential $V_{0}\left(r_{3}\right)$ of figure $(2 a)$.

The calculations were performed at a relative kinetic energy of $4.23 \mathrm{eV}$ and c.m. scattering angles of $49.2^{\circ}$ and $37.1^{\circ}$. The two-body t-matrix elements for the exponential repulsive potential were computed using a propagating algorithm due to Brumer and Shapiro [15].

The following approximation was introduced in order to simplify the DWIA computations. We note that the quantities $S_{l_{1}}\left(p_{3}, p_{3 u}\right)$ appearing in the integral on the right side of eq. (30) are defined by, 
$S_{\ell_{1}}\left(p_{3}, p_{3 \alpha}\right)=\frac{\delta\left(p_{3}-p_{3 \alpha}\right)}{p_{3 \alpha}^{2}}+\frac{2 \mu_{3}}{h^{2}} \lim _{i \rightarrow 0^{*}} \frac{t_{0 \ell_{1}}\left(p_{3}, p_{3 a}\right)}{p_{3 a}^{2}-p_{3}^{2}+i \varepsilon}$

and

$\lim _{u^{(}}\left(p_{3 \mu}^{2}-p_{3}^{2}+i \varepsilon\right)^{-1}=\frac{\rho}{p_{3 \alpha}^{2}-p_{3}^{2}}-\pi i \delta\left(p_{3 \mu}^{2}-p_{3}^{2}\right)$

where $\delta$ is the Dirac delta function and $P$ denotes the principal value integral. We now approximate the limit by taking only the delta function term, and ignoring the principal value term, on the right side of the above equation. Recilling the equivalence of the distorted wave formalism and the multiple collision serics expansion (eqs. (12) and (13)), this approximation corresponds to keeping only the on-shell, energy-conserving, terms in the transition matrix $T_{0}$ due to scattering from the spherically symmetric potential $V_{0}\left(r_{3}\right)$. This approximation conserves energy at the first and the third of the three step collision process. For this reason it can also be regarded as "classical" approximation. Of course, a completely classical calculation would conserve energy at every step of the collision process. The quantities $S_{f_{1}}\left(p_{3}, p_{3 \alpha}\right)$ can then be approximated by,

$S_{\ell_{1}}\left(p_{3}, p_{3 \alpha}\right) \approx \frac{\delta\left(p_{3}-p_{3 u}\right)}{p_{3 u}^{2}}-\pi i \frac{\mu_{3}}{h^{2} p_{3 u}} t_{0 \ell_{1}}\left(p_{3}, p_{3 a}\right)\left[\delta\left(p_{3}+p_{3 u}\right)+\delta\left(p_{3}-p_{3 u}\right)\right]$.

A similar expression is obtained for $S_{\ell_{1}}\left(p_{3}^{\prime}, p_{3 \beta}\right)$. The DWIA indastic c.m. differential cross section is then given by eqs. (34) and (35) with the quantities $g_{\mu_{1} \ell_{i}^{\prime}}^{(0)}\left(v^{\prime} j^{\prime} p_{3 \beta} ; v j p_{3 u}\right)$ on the right side of eq. (35) approximated by,

$$
\begin{aligned}
& b_{3 \ell_{1} \ell_{i}}^{(3)}\left(v^{\prime} j^{\prime} p_{3 \beta} ; v j p_{3 \alpha}\right) \approx \\
& \left(1-\pi p_{3 \alpha} \frac{\mu_{3}}{\hbar^{2}} t_{0 \ell_{1}}\left(p_{3 \alpha}, p_{3 \alpha}\right)\right) A_{3 \ell_{1} \ell_{i}}^{(0)}\left(v^{\prime} j^{\prime} p_{3 \beta} ; v j p_{3 \alpha}\right)\left(1-\pi i p_{3 \beta} \frac{\mu_{3}}{\hbar^{2}} t_{0 \ell_{i}}\left(p_{3 \beta}, p_{3 \beta}\right)\right),
\end{aligned}
$$


where the on-shell two-body t-matrices $t_{0 f}(k, k)=\sin \delta_{0 \ell} \exp \left(i \delta_{0 \ell}\right)$ are calculated from eq. (27) using the method of Brumer and Shapiro [15]. Eq. (A27) in the appendix was used to generate $A_{j l_{1} f_{1}}^{(0)}\left(v^{\prime} j^{\prime} p_{3 \beta} ; v j p_{3 u}\right)$. The quantities $F_{J M_{1}}^{(0)}\left(v^{\prime} j^{\prime} p_{3 \beta} ; v j p_{3 u}\right)$ required on the right hand side of eq. (A27) are computed, from eq. (A24), using the exact IA calculation, or from eq. (A32), using the part-classical IA calculation [1].

\section{RESULTS}

The IA differential cross sections calculated at a relative kinetic energy of $4.23 \mathrm{eV}$ and a c.m. scattering angle of $49.2^{\circ}$ are plotted as a function of final rotational quantum number $j^{\prime}$ in figures (3a) and ( $\left.3 b\right)$ for the hard core and the exponential repulsive atomatom interactions, respectively; the DWIA differential cross sections are plotted in figure (3c). The experimental differential cross sections [10] were normalized so that the value for the most probable final rotational level $j^{\prime}$ in figure (3c) agreed with the largest calculated DWIA differential cross section. Thereafter the comparisons of the differential cross sections are absolute. The very same calculations are repeated for the c.m. scattering angle of $37.1^{\circ}$ and the results plotted in figures (4a), (4b), and (4c). For both angles, the calculated IA distributions are narrower and peak at a larger final rotational level $\mathrm{j}^{\prime}$ than the experimentally observed distribution [10], also shown in the figures. The calculated differential cross sections using either of the IA atom-atom potential functions, the exponential functions, eq. (37), or the hard core functions, eq. (38), are remarkably similar. From these results it would appear that the IA results are insensitive to the shape of the repulsive portion of the atom-atom potential. This realization, coupled with the computational easc, is the reason for choosing hard core functions, equation (38), together with the distorting potential, figure (2a), as the total 
atom-diatom potential $\mathrm{V}$ for our DWIA calculation. The DWIA results reprevent an improvement over the IA results. The DWIA distributions are broader and also peak at a lower final rotational level $j$, in much better agreement with the experimentally observed distributions.

These results may be interpreted as follows: The long range centrally symmetric potential $V_{0}\left(r_{3}\right)$ perturbs the incoming and outgoing waves by giving them a partial wave dependent phase shift. The transition amplitudes from different partial waves no lunger add coherently, leading to a broadening of the calculated distribution of differential cross sections. This broadening also leads to smaller momentum transfer shifting the maximum of the calculated distribution closer to the maximum of the mestsured distribution. The more realistic DWIA potential leads to a better agreement of the calculated differential cross sections with the measured ones.

The part-classical IA calculation has been used in our recent work $[4,5]$ to reduce the computation time. It was estimated that this approximation introduces an error of about $10 \%$ in the calculation. The present work, because it involves low rotational quantum numbers, may be considered a stringent test of the part-classical calculation. Table 1 gives the ratio of the part-classical and the exact DWIA differential cross sections. It is seen that for final rotational quantum numbers greater than 10 , the accuracy of the approximate part-classical method is indeed about $10 \%$.

Figure (5) compares the IA and DWIA calculations for larger scattering angles; the collision parameters are the same as in figures (3) and (4). Although there are differences in the magnitude of the cross sections, the IA and DWIA rotational distributions for large c.m. scattering angles are nearly the same. We have now a quantitative estimate of the influence of the long range attractive potential on the inelastic cross sections when only a small amount (less than $25 \%$ for the most probable rotational transition) of the initial relative translational energy is converted into rotational 
energy. Of course, this influence is expected to be a function of the initial and final relative translational energies as well as the initial state of the diatom.

\section{SUMMARY}

The improved agreement between the DWIA calculated distributions of rotational transition probabilities and the experimentally derived ones suggest that the long range attractive part of the potential must be included in a model for small angle, high energy, atom-diatom collisions involving small amounts of energy transfer. These results, which appear reasonable, warrant further investigation (additional calculations at different relative kinetic energies, scattering angles, and on different systems) to test the consistency of the model. The significance of neglecting the multiple collision terms and the principal part on the right side of eq. (41) has yet to be investigated. The present calculation, however raises the hope that an accurate calculation of the differential cross sections of inelastic processes at high energies may be possible using a suitable modification of the impulse approach. As expected, the IA and DWIA calculations give nearly the same rotational distributions for large scattering angles.

This investigation was in part funded by AFOSR under the Geophysics Scholar program. The authors gratefully acknowledge the help of Mr. J. Sindoni with the computations. This research was also in part supported by AFOSR under task 2303 EP, project 007. The authors are grateful to Dr. J. Peter Toennies for taking the time from his busy schedule to check that the results of his group were being correctly cited and make useful comments on the manuscript. 


\section{FIGURE CAPTIONS}

Figure 1. $\mathrm{Li}^{+}-\mathrm{N}_{2}$ interaction potential as a function of $\mathrm{r}_{3}$ for the collinear $\left(\alpha=0^{\circ}\right)$ and $\mathrm{C}_{2 \mathrm{v}}\left(\alpha=90^{\circ}\right)$ configurations. The points shown are ab initio SCF energies (Staemmler [13]). Also shown are lines resulting from approximations by a sum of exponential pair

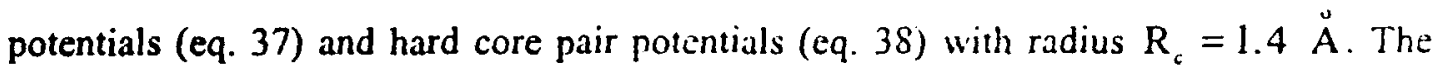
molecule equilibrium distance, $1.1 \AA$, is taken as the intemuclear separation .

Figure 2. (a) Central symmetric potential $V_{0}\left(r_{3}\right)$ used in DWIA calculations.

(b) Total interaction potential used in DWIA calculations, resulting from a sum of hard core pair potentials $V_{1}\left(y_{1}\right)+V_{2}\left(y_{2}\right)\left(R_{c}=1.4 \AA ् A\right)$ and $V_{v}\left(r_{3}\right)$ (eq. 39), shown for the collinear configuration $\left(\alpha=0^{\circ}\right)$. The points shown are ab initio SCF energies (Staemmler [13]).

(c) Same as in fig. $2 b$, for the $C_{2 v}$ configuration.

Figure 3. Distributions of rotational transition probabilities, shown as c.m. rotationally inelastic differential cross sections vs final rotational quantum number $j$, for $\mathrm{Li}^{+}+\mathrm{N}_{2}(0,2) \rightarrow \mathrm{Li}^{+}+\mathrm{N}_{2}\left(0 \mathrm{j}^{\prime}\right)$ at a relative kinetic energy of $4.23 \mathrm{eV}$ and a c.m. scattering angle of $\theta=49.2^{\circ}$. The calculated differential cross sections are represented by discrete sticks. The solid line is the experimentally derived distribution [10]. Top frame, figure (3a) is the exact IA calculation, using hard core pair interactions (eq. 38) with radius $R_{c}=1.4 \AA$. Middle frame, figure (3b), is the exact IA calculation, using exponential pair interactions (eq. 37). Bottom frame, figure (3c), is the DWIA calculation, using the potential shown in figures (2b) and (2c). The measured differential cross sections are relative and are normalized by selting the largest measured cross section equal to the largest DWIA differential cross section. The experimental cross 
sections are measured for a tixed laboratory scultering angle. The cilted c.nn. scattcring angle is only for elastic scattering. The c.m. scattering angle is different for each rotational transition; for $\mathrm{j}^{\prime}=30$ the c.m. scattering angle is about $49.5^{\circ}-0.3^{\circ}$ degree larger than the value cited for elastic scattering. These small differences are neglected in the present work.

Figure 4. Same as fig.3, for a c.m. scaltering angle of $\theta=37.1^{\circ}$.

Figure 5. The IA (solid line) and DWIA ( discrete sticks) calculations for the collision parameters in figure (3) are plotted for $60^{\circ}, 90^{\circ}, 120^{\circ}$, and $150^{\circ} \mathrm{c} . \mathrm{m}$. scattering angles. The two distributions are nearly the same for larger scattering angles. The shift of the rotational distribution with increasing value of the c.m. scattering angle to larger values of the final rotational quantum numbers accompnied by decreasing differential scattering cross section is to be noted. 
REFFRENCES

1. R.D. Sharma, P.M. Bakshi and J.M. Sindoni, "Impulse Formalism for Atom-Diatom Collisions," Phys. Rev. A 43: 189 (1991).

2. R.D. Sharma, P.M. Bakshi and J.M. Sindoni, "Impulse Formalism for Atom-Molecule Collisions: Inadequacy of the Peaking Approximation," Phys. Rev. A 40: 1692 (1989).

3. R.D. Sharma, P.M. Bakshi and J.M. Sindoni, "Criteria for Applicability of the Impulse Approach to Collisions," Phys. Rev. A 41: 6540 (1992).

4. R.D. Sharma and J.M. Sindoni, "Relaxation of Highly Vibrationally Excited KBr by Ar," Phys. Rev. A 45: 531 (1992).

5. J. M. Sindoni and R.D. Sharma, "Mechanism of Ballistic Collisions," Phys. Rev. A 45: R2659 (1992).

R.D. Sharma and J.M. Sindoni, "Inelastic and Ballistic Processes Resulting from CsF-Ar Collisions," J. Chem. Phys. 97: 737 (1992).

6. L.H. Beard and D.A.Micha, "Collision Dynamics of Three Interacting Atoms: Vibrational-Rotational Excitation in AtomDiatom Hyperthermal Collisions," J. Chem. Phys. 74: 6700 (1981).

7. L.I. Schiff, Quantum Mechanics, Mc Graw-Hill Book Company, New York, 1968 .

8. J.M.Eisenberg and D.S. Kolton, Theory of Meson Interactions with Nuclei, John Wiley and Sons, New York, 1980.

9. P. Eckelt, H.J. Korsch and V. Philipp, "Energy Loss Spectra for Vibro-Rotational Molecular Excitation in an Impulsive Spectator Model," J. Phys. B 7: 1649 (1974).

10. R. Bottner, U. Ross and J. Peter Toennies, "Measurements of Rotational and vibrational Excitation in the scattering of $\mathrm{Li}^{+}$from $\mathrm{N}_{2}$ and $\mathrm{CO}$ at Center of Mass Energies of 4.23 and $7.07 \mathrm{eV}, "$ J. Chem. Phys. 110: 115 (1984).

11. U. Gierz, J. Peter Toennies and M. Wilde, "A New Look at Rotational and Vibrational Excitation in the Scattering of $\mathrm{Li}^{+}$ from $\mathrm{N}_{2}$ and $\mathrm{CO}$ at Energies Between 4 and $17 \mathrm{eV}, "$ Chem. Phys. Lett. 110: 115 (1984).

12. V. Philipp, H.J. Korsch and P. Eckelt, "Inelastic Atom-Diatom Molecule Collisions: II. Influence of Initial Vibration- 
al and Rotational Excitation", J. Phys. B 10: 117 (1977).

13. V. Staemmler, "AB Initio Calculation of the Potential Energy Surface of the System $\mathrm{N}_{2} \mathrm{Li}^{+}$," Chem. Phys. 7: 17 (1975).

14. E.A. Gislason, P. Polak-Dingels and M.S. Rajan, "Determination of the spherically symmetric Potential components for $\mathrm{Li}^{+}-\mathrm{N}_{2}$ and $\mathrm{Li}^{+}-\mathrm{CO}$ from Total Cross Section Measurements," J. Chem. Phys. 93: 2476 (1990).

15. P. Brumer and M. Shapiro, "Multiple Scattering Theory: I. off-Shell $T$ Matrix Elements and Momentum Space Wavefunctions for Local Two Body Potentials," J. Chem. Phys. 63: 427 (1975).

16. R.N. Zare, Anqular Momentum, Wiley-Interscience, New York, 1988 . 


\section{APPENDIX}

In this appendix, we shall derive the expression for the IA transition amplitude $\left\langle v^{\prime} j^{\prime} m^{\prime} p_{3}^{\prime}\left|T^{(s)}\right| v j m p_{3}\right\rangle$ in terms of the momenta $\rho_{3}^{\prime}$ and $\boldsymbol{\rho}_{3}$. The scattering process involves the collision of atom 3 with a molecule consisting of atoms 1 and 2. We again make use of the set of Jacobi momenta, where in a system of three particles, $c$ incident on diatom ab, $\mathbf{p}_{\mathbf{c}}$ denotes the momentum of $\mathrm{c}$ with respect to the center of mass (c.m.) of $a b$; and $\mathbf{q}_{c}$ is the relative momentum of $a$ and $b$. We also use unprimed and primed notations to denote momentum vectors before and after the collision, respectively. Following SBS, the IA transition amplitude with momentum transfer $q$ can be written as,

$$
\left\langle\phi_{3}^{\prime}\left|T^{(\boldsymbol{s})}(\mathbf{q})\right| \phi_{3}\right\rangle=\int \mathrm{d} \mathbf{q}_{3} \phi^{\prime *}\left(\mathbf{q}_{3}^{\prime}\right)\left\langle\mathbf{q}_{.}^{\prime}\left|t^{(s)}(\mathbf{q})\right| \mathbf{q}_{3}\right) \phi\left(\mathbf{q}_{3}\right), \quad s=1,2
$$

where $q \equiv p_{3}^{\prime}-p_{3},\left|\phi_{3}\right\rangle \equiv\left|\Psi_{v j n} p_{3}\right\rangle,\left|\phi_{3}^{\prime}\right\rangle \equiv\left|\psi_{v{ }^{\prime} j m^{\prime}}, p_{3}^{\prime}\right\rangle, s$ is the spectator atom, and $\phi\left(q_{3}\right)$ is the initial-state wave function of the molecule in the momentum representation:

$\phi\left(\mathbf{q}_{3}\right)=(2 / \pi)^{1 / 2} i^{j} I_{v j}\left(q_{3}\right) Y_{j m}\left(\hat{\mathbf{q}}_{3}\right)$

where,

$I_{v j}\left(q_{3}\right)=\int_{0}^{\infty} y_{3} d y_{3} \chi_{v j}\left(y_{3}\right) j_{j}\left(q_{3} y_{3}\right)$

Eq. (Ala) was obtained by applying the IA, i.e., reducing the three-body Moller operator to the two-body Møller operator by approximating the intramolecular potential energy operator by a number, and by assuming that one of the two atoms of the molecule acts as a spectator during the collision. Additional details of the derivation of eq. (Ala) are given in SBS. 
We first note that the final-state wave function in the momentum and coordinate representations are related by the Fourier transform

$\phi^{\prime *}\left(q_{3}^{\prime}\right)=(2 \pi)^{-3 / 2} \int d y_{3} \exp \left(-i q_{3} \cdot y_{3}\right) \exp \left(-i \alpha_{0}\left(p_{3}^{\prime}-p_{3}\right) \cdot y_{3}\right) \psi^{\prime *}\left(y_{3}\right)$

where we have used the relation, $\mathbf{q}_{3}^{\prime}=\mathbf{q}_{3}+\alpha_{3} \mathbf{q}=\mathbf{q}_{3}+\alpha_{3}\left(\mathbf{p}_{3}^{\prime}-\mathbf{p}_{3}\right)$ and $\alpha_{4}$ is a mass ratio defined by $\alpha_{1}=(-1)^{\circ} \frac{m_{1}}{m_{1}+m_{2}}$. Eq. (Ala) may then be rewritten as:

$\left\langle v^{\prime} j^{\prime} m^{\prime} \mathbf{p}_{3}^{\prime}\left|T^{(0)}\right| v j m p_{3}\right\rangle=\int d y_{3} \psi^{\prime *}\left(y_{3}\right) \exp \left(-i \alpha_{1}\left(p_{3}^{\prime}-p_{3}\right) \cdot y_{3}\right) \Psi^{(s)}\left(y_{3}\right)$

In equation (Alb), $\Psi^{\prime}\left(\mathrm{y}_{3}\right) \equiv \Psi_{v^{\prime} j^{\prime \prime} n^{\prime}}\left(\mathrm{y}_{3}\right)$ is the vibrational-rotational wave function for the final-state of the diatom,

$\psi^{\prime}\left(\mathbf{y}_{3}\right) \equiv \chi_{v^{\prime j}}\left(y_{3}\right) Y_{j^{\prime} m^{\prime}}\left(\hat{y}_{3}\right)$

where $\chi_{v j^{\prime}}\left(y_{3}\right)$ is the wavefunction for the vibrational motion and the $Y_{j^{\prime} m^{\prime}}\left(\hat{y}_{3}\right)$ is the spherical harmonic representing the wavefunction for the rotational motion. The function $\Psi^{(0)}\left(y_{3}\right)$ is defined as the Fourier transform of the initial-state wavefunction, in the momentum representation, modified by the effect of the collision,

$\Psi^{(0)}\left(\mathbf{y}_{3}\right) \equiv(2 \pi)^{-3 / 2} \int \mathrm{dq} q_{3} \operatorname{ex}\left(-\mathrm{iq}_{3} \cdot \mathbf{y}_{3}\right)\left\langle\mathbf{q}^{\prime}\left|\mathrm{t}^{(0)}\right| \mathbf{q}_{\mathbf{3}}\right\rangle \phi\left(\mathbf{q}_{3}\right)$

The dependence of the two-body $t^{(\bullet)}$ matrix element on the orientation of $\hat{\mathbf{q}}_{3}, \hat{\mathbf{p}}_{3}$ and $\hat{\mathbf{p}}_{\text {, }}^{\prime}$ is factored out by writing, 


$$
\begin{aligned}
& \left\langle\mathbf{q}_{.}^{\prime}\left|\mathbf{t}^{(0)}\right| \mathbf{q}_{\mathbf{0}}\right\rangle=\sum_{L, M} \mathrm{~T}_{\mathrm{L}_{M}}^{(0)}\left(\mathbf{q}_{3}, \mathbf{p}_{3}, \mathbf{p}_{3}^{\prime}\right) \mathrm{Y}_{\mathrm{IM}}\left(\hat{\mathbf{q}}_{3}\right)
\end{aligned}
$$

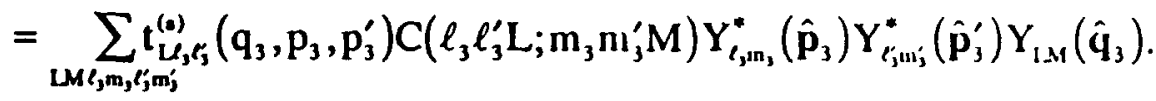

It is possible to write equation (A6) because the t-matrix element, a scalar, depends upon three vectors $\mathbf{p}_{3}, \mathbf{p}_{3}^{\prime}$, and $\mathbf{q}_{3}$ which must couple only in ways that the resultant expression is also a scalar. Substituting the expansion on the second line of ey. (At) into eq. (A5) and using the plane wave expansion,

$\exp \left(-\mathbf{i q}_{3} \cdot \mathbf{y}_{3}\right)=4 \pi \sum_{\hat{i}}(-i)^{\lambda} j_{\lambda}\left(q_{3} y_{3}\right) \sum_{\mu} Y_{\lambda \mu}^{*}\left(\hat{\mathbf{q}}_{3}\right) Y_{\lambda \mu}\left(\hat{\mathbf{y}}_{3}\right)$

we obtain

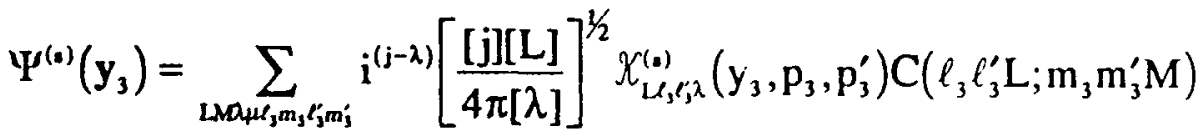

$$
\begin{aligned}
& \times \mathrm{C}(\mathrm{jL} \lambda ; 000) \mathrm{C}(\mathrm{jL} \lambda ; \mathrm{mM} \mu) \mathrm{Y}_{\lambda \mu}\left(\hat{\mathbf{y}}_{3}\right) \mathrm{Y}_{\ell, \mathrm{m}, \mathrm{j}}^{*}\left(\hat{\mathbf{p}}_{3}\right) \mathrm{Y}_{\ell_{\mathrm{j}, \mathrm{wj}}^{*}}^{*}\left(\hat{\mathbf{p}}_{3}^{\prime}\right) \text {, }
\end{aligned}
$$

where,

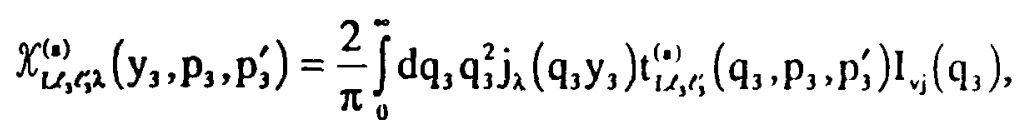

and $[\mathrm{j}] \equiv(2 \mathrm{j}+1)$. Now using eqs. (A4) , (A8) and the plane-wave expansions,

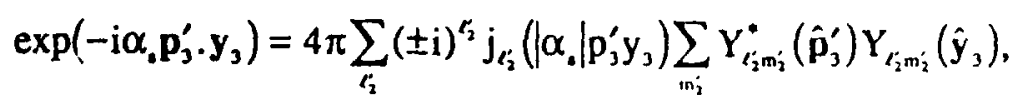

and 
$\exp \left(i \alpha, p_{3} \cdot y_{3}\right)=4 \pi \sum_{\iota_{2}}( \pm i)^{-\iota_{2}} j_{\ell_{2}}\left(|\alpha \cdot| p_{3} y_{3}\right) \sum_{m_{2}} Y_{\iota_{2} m_{2}}\left(\hat{\mathbf{p}}_{3}\right) Y_{i_{2} m_{2}}^{*}\left(\hat{y}_{3}\right)$

where \pm means that the plus and minus signs are taken for $s=1$ and $s=2$, respectively. The angular integral in equation (Alb) is

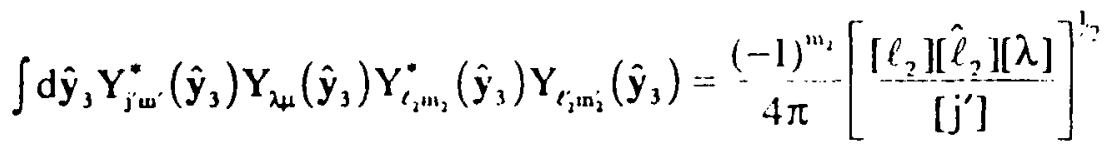

$$
\begin{aligned}
& \times \sum_{L^{\prime} M^{\prime}} C\left(\ell_{2} \ell_{2}^{\prime} L^{\prime} ; 00\right) C\left(\lambda L^{\prime} j^{\prime} ; 00\right) C\left(\ell_{2} \ell_{2}^{\prime} L^{\prime} ;-m_{2} m_{2}^{\prime} M^{\prime}\right) C\left(\lambda L^{\prime} j^{\prime} ; \mu M^{\prime} m^{\prime}\right) \text {. }
\end{aligned}
$$

The transition amplitude of eq. (Alb) can now be written as

$\left\langle v^{\prime} j^{\prime} m^{\prime} \mathbf{p}_{3}^{\prime}\left|T^{(s)}\right| v j m p_{3}\right\rangle=$

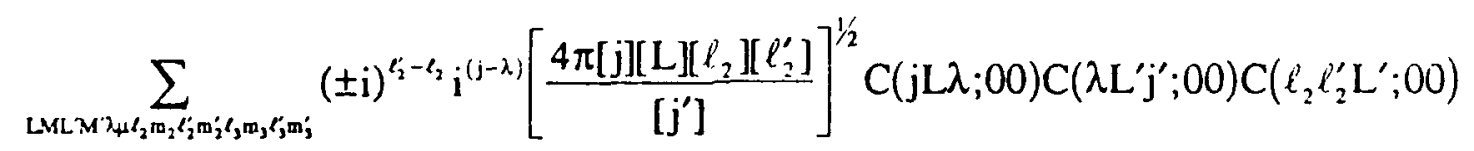

$\times C(j L \lambda ; m M \mu) C\left(\lambda L^{\prime} j^{\prime} ; \mu M^{\prime} m^{\prime}\right) C\left(\ell_{2} \ell_{2}^{\prime} L^{\prime} ; m_{2} m_{2}^{\prime} M^{\prime}\right) C\left(\ell_{3} \ell_{3}^{\prime} L ; m_{3} m_{3}^{\prime} M\right)$

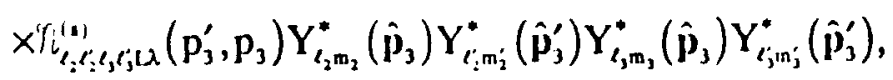

where,

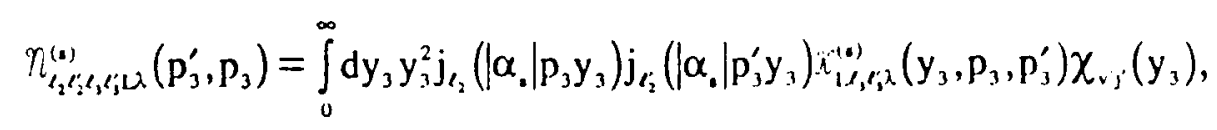

In obtaining eq (A13), we have used the relation

$$
Y_{i_{2}-m_{2}}^{\cdot}\left(\hat{\mathbf{p}}_{3}\right)=(-1)^{\mathbf{m}_{2}} \mathbf{Y}_{\ell_{2} m_{2}}\left(\hat{\mathbf{p}}_{3}\right)
$$

Eq. (A13) gives an expression for the transition amplitude in terms of the momenta $\mathbf{p}_{3}$ and $\mathbf{p}_{3}^{\prime}$. A simpler expression can be obtained by using the relations, 


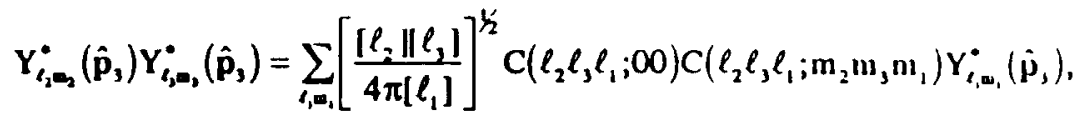

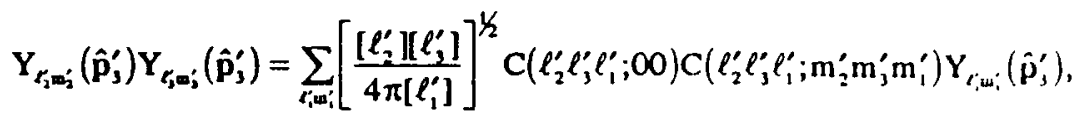

$$
\begin{aligned}
& \sum_{\mu} \mathrm{C}(\mathrm{jL} \lambda ; \mathrm{mM} \mu) \mathrm{C}\left(\lambda \mathrm{L}^{\prime} \mathrm{j}^{\prime} ; \mu \mathrm{M}^{\prime} \mathrm{m}^{\prime}\right)= \\
& \sum_{j M_{1}}[[\lambda][J]]^{1 / 2} W\left(j L j^{\prime} L^{\prime} ; \lambda J\right) C\left(j j^{\prime} ; m M_{1} m^{\prime}\right) C\left(L L^{\prime} J ; M M^{\prime} M_{1}\right) \text {, }
\end{aligned}
$$

and substituting into (A13). We then obtain

$$
\begin{aligned}
& \left\langle v^{\prime} j^{\prime} m^{\prime} \mathbf{p}_{3}^{\prime}\left|T^{(s)}\right| v_{j m p_{3}}\right\rangle=
\end{aligned}
$$

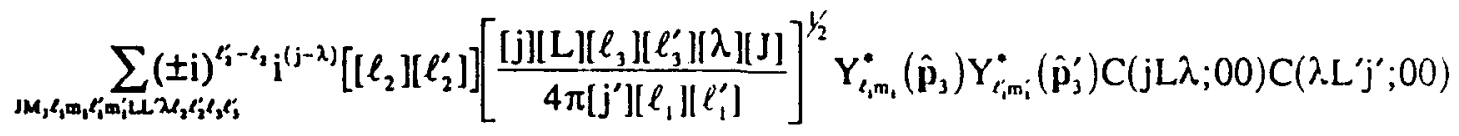

$$
\begin{aligned}
& \times \mathrm{C}\left(\ell_{2} \ell_{2}^{\prime} \mathrm{L}^{\prime} ; 00\right) \mathrm{C}\left(\ell_{2} \ell_{3} \ell_{1} ; 00\right) \mathrm{C}\left(\ell_{2}^{\prime} \ell_{3}^{\prime} \ell_{;}^{\prime} ; 00\right) \mathrm{C}\left(\mathrm{jJj} ; \mathrm{mM}, \mathrm{m}^{\prime}\right) \mathrm{W}\left(\mathrm{jLj} \mathrm{j}^{\prime} \mathrm{L}^{\prime} ; \lambda J\right) \\
& \left.\times \sum_{M M^{\prime} m_{2} m_{i}^{\prime}, m_{3}^{\prime}} \mathrm{LL} J ; M M^{\prime} M_{3}\right) C\left(\ell_{2} \ell_{2}^{\prime} L^{\prime} ; m_{2} m_{2}^{\prime} M^{\prime}\right) C\left(\ell_{3} \ell_{3}^{\prime} L ; m_{3} m_{3}^{\prime} M\right) \\
& \times C\left(\ell_{2} \ell_{3} \ell_{1} ; m_{2} m_{3} m_{1}\right) C\left(\ell_{2}^{\prime} \ell_{3}^{\prime} \ell_{1}^{\prime} ; m_{2}^{\prime} m_{3}^{\prime} m_{1}^{\prime}\right) \times \eta_{l_{1}, l_{2}^{\prime}, r_{1}, u}^{(2)}\left(p_{3}, p_{3}^{\prime}\right) \text {. }
\end{aligned}
$$

Noting that the sums $\ell_{2}+\ell_{3}+\ell_{1}$ and $\ell_{2}^{\prime}+\ell_{3}^{\prime}+\ell_{1}^{\prime}$ must be even for a nonvanishing contribution, the sum over the product of five Clebsch-Gordan coefficients on the right side of the above equation is equal to

$\left[\left[\ell_{1}\right]\left[\ell_{1}^{\prime}\right][L]\left[L^{\prime}\right]\right]^{1 / 2} \mathrm{C}\left(\ell_{1} \ell_{1}^{\prime} \mathrm{J} ; \mathrm{m}_{1} \mathrm{~m}_{1}^{\prime} \mathrm{M}_{1}\right) \mathrm{X}\left(\ell_{1} \ell_{1}^{\prime} \mathrm{J} ; \ell_{3} \ell_{3}^{\prime} L ; \ell_{2} \ell_{2}^{\prime} L^{\prime}\right)$,

where $\mathrm{X}$ is a 9-j symbol. We finally obtain the expression for the transition amplitude 
$\left\langle v^{\prime} j^{\prime} m^{\prime} \mathbf{p}_{3}^{\prime}\left|T^{(0)}\right| v j m p_{3}\right\rangle=$

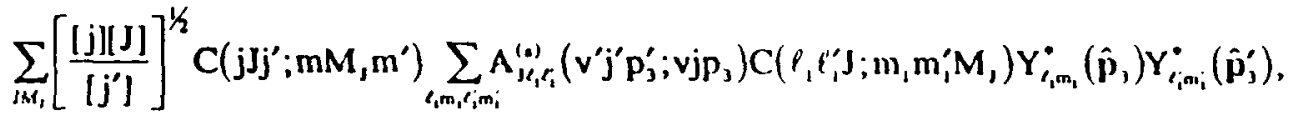

where,

$$
\begin{aligned}
& A_{3 t_{1} i_{i}^{\prime}}^{(3)}\left(v^{\prime} \mathrm{j}^{\prime} \mathrm{p}_{3}^{\prime} ; \mathrm{vjp}_{3}\right)=
\end{aligned}
$$

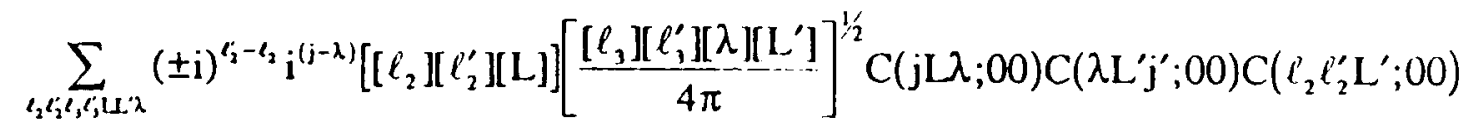

$$
\begin{aligned}
& \times \mathrm{C}\left(\ell_{2} \ell_{3} \ell_{1} ; 00\right) \mathrm{C}\left(\ell_{2}^{\prime} \ell_{3}^{\prime} \ell_{1}^{\prime} ; 00\right) \mathrm{W}\left(\mathrm{jLj} \mathrm{J}^{\prime} ; \lambda \mathrm{J}\right) \times\left(\ell_{1} \ell_{1}^{\prime} \mathrm{J} ; \ell_{3} \ell_{3}^{\prime} \mathrm{L} ; \ell_{2} \ell_{2}^{\prime} \mathrm{L}^{\prime}\right)
\end{aligned}
$$

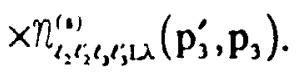

Equations (A21) and (A22) give the expression for the IA collision amplitude in terms of the momenta $\mathbf{p}_{3}$ and $\mathbf{p}_{3}^{\prime}$. The equivalent expression in terms of the momentum transferred $\mathbf{q}=\mathbf{p}_{3}^{\prime}-\mathbf{p}_{3}$ was given in SBS:

$$
\left\langle v^{\prime} j^{\prime} m^{\prime} p_{3}^{\prime}\left|T^{(0)}\right| v j m p_{3}\right\rangle=\sum_{M_{1}}\left[\frac{[j][J]}{\left[j^{\prime}\right]}\right]^{1 / 2} C\left(j J^{\prime} ; m M_{3} m^{\prime}\right) F_{M_{1}}^{(0)}\left(v^{\prime} j^{\prime} p_{3}^{\prime} ; v j p_{3}\right),
$$

where,

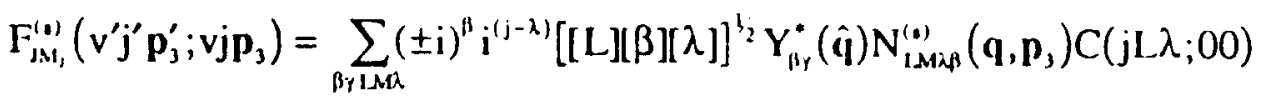

$$
\begin{aligned}
& \times C\left(\lambda \beta j^{\prime} ; 00\right) C\left(L \beta J ; M \gamma M_{1}\right) W\left(j L j^{\prime} \beta ; \lambda J\right),
\end{aligned}
$$

with,

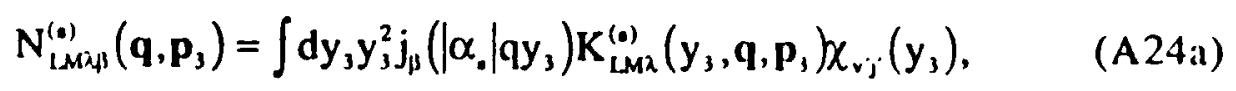


$K_{L M \lambda}^{(\cdot)}\left(y_{3}, q, p_{3}\right)=\frac{2}{\pi} \int_{0}^{\infty} d q_{3} q_{3}^{2} j_{\lambda}\left(q_{3} y_{3}\right) T_{i M}^{(\bullet)}\left(q_{3}, p_{3}, p_{3}^{\prime}\right) I_{v j}\left(q_{3}\right)$

and $T_{L M}^{(\bullet)}\left(q_{3}, p_{3}, p_{3}^{\prime}\right)$ defined by the expansion on the first line of eq. (AG). Both expressions give the exact same numerical results. $A$ comparison of eqs (A21) and (A23) leads to the relation:

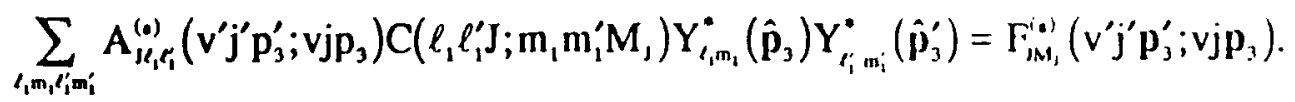

Eq. (A25) provides an alternative way to obtain the quantitics $A_{\mu_{1} \ell_{1}^{\prime}}^{\left({ }^{\prime}\right)}\left(v^{\prime} \mathrm{j}^{\prime} p_{3}^{\prime} ; \mathrm{vjp} p_{3}\right)$. Since these quantities are not dependent on the magnetic quantum numbers, they may be evaluated in any coordinate system. Taking the z-axis along the direction of $p_{3}$, we have

$Y_{\ell_{1} \mathrm{~m}_{1}}^{*}\left(\hat{\mathbf{p}}_{3}\right)=\left[\frac{\left[\ell_{1}\right]}{4 \pi}\right]^{1 / 2} \delta_{\mathrm{m}_{1}, 0}$,

and the quantities $A_{j l_{1} e_{1}}^{(o)}\left(v^{\prime} j^{\prime} p_{3}^{\prime} ; v_{j p}\right)$ can now be obtained by the relation,

$$
\begin{aligned}
A_{3 \ell_{1} \ell_{i}}^{(\bullet)}\left(v^{\prime} j^{\prime} p_{3}^{\prime} ; v_{j p}\right)= & (-1)^{\ell_{1}}\left[\frac{[J]}{4 \pi}\right]^{-1 / 2} \sum_{M_{1}} C\left(J \ell_{1}^{\prime} \ell_{1} ;-M_{3} M_{1} 0\right) \\
& \times \int F_{J M_{1}}^{(a)}\left(v^{\prime} j^{\prime} \mathbf{p}_{3}^{\prime} ; v j p_{3} ; \hat{\mathbf{z}}=\hat{\mathbf{p}}_{3}\right) Y_{\ell_{1}^{\prime}-M_{1}}^{*}\left(\hat{\mathbf{p}}_{3}^{\prime}\right) \mathrm{d} \hat{\mathbf{p}}_{3}^{\prime},
\end{aligned}
$$

where the more specific notation $\mathrm{F}_{\mathrm{M}}^{(\mathbf{s})}\left(\mathrm{v}^{\prime} \mathrm{j}^{\prime} \mathbf{p}_{3}^{\prime} ; \mathrm{vjp}_{3} ; \hat{\mathbf{z}}=\hat{\mathbf{p}}_{3}\right)$ indicates that these quantities have been calculated in a coordinate system with the z-axis of the coordinate system taken along the direction of $\mathbf{p}_{\mathbf{3}}$. That can be accomplished in a straightforward manner using the method outlined in SBS, i.e., eq. (A24). Eq. (A27) provides a much more efficient algorithm to obtain $A_{u_{1} e_{i}}^{(0)}\left(v^{\prime} j^{\prime} p_{3}^{\prime} ; v_{j p}\right)$ than eq. (A22), where summations over 
very large quantum numbers are called for. Once available, these quantities can be used to compute the collision amplitude as given by eq. $(\wedge 21)$.

A part-classical (PC) approximation was introduced in SBS for a computationally efficient evaluation of the IA collision amplitude when the $z$-axis of the coordinate system is taken along the direction of the momentum transfer vector $\mathbf{q}=\mathbf{p}_{3}^{\prime}-\mathbf{p}_{3}$. Without going into all the details already given in SBS, this approximation involves evaluating the two-body $\mathrm{t}^{(\bullet)}$ matrix in eqs. (Ala) and (Alb) using classical mechanics. The function $\Psi^{(\bullet)}\left(y_{3}\right)$ defined in eq. (AS) is then replaced by $t_{1 x}^{(\infty)} \Psi\left(y_{3}\right)$ where $\psi\left(y_{3}\right)$ is the initial-state molecular vibrational-rotational wave function in coordinate representation and $t_{P C}^{(0)}$ is the two-body $t^{(+)}$matrix evaluated at a value of internal momentum, denoted $q_{3}^{\text {icc }}$, chosen so that $q_{a}^{\prime}=q_{\text {. }}$, i.e., an on-the-encrgy-shell twobody $\mathbf{t}^{(0)}$ matrix is obtained:

$t_{P C}^{(0)} \equiv\left[\left\langle\mathbf{q}_{1}^{\prime}\left|t^{(0)}\right| \mathbf{q}_{1}\right\rangle\right]_{\left.\mathbf{q}_{3}=\mathbf{q}_{1}\right)^{\prime c}}$

As specified in SBS, the $x$ and $y$ components of $\mathbf{q}_{3}^{\mathrm{PC}}$ are set equal to zero and its $z$ component (along $\mathbf{q}$ ) is given by:

$\mathrm{q}_{32}^{\mathrm{PC}}=-\frac{\alpha, \mathrm{q}}{2}+\frac{\mu_{12} \Delta \varepsilon}{\alpha_{.9}}$

where $\mu_{12}$ is the reduced mass of the molecule and $\Delta \varepsilon$ is the difference in internal energy between its initial and final states. The PC approximation was shown in SBS to correspond to a situation in which the z-component of the incident momentum $q$. changes sign while the $x$ and $y$ components are unaffected. This is the situation which arises during a collision between two hard spheres in classical mechanics when the $z$ axis is taken along the direction normal to the surface of the two spheres at the point of contact. Since the hard sphere two-body $t^{(s)}$ matrix is still evaluated using quantum 
mechanics, this approximation was called "part classical". The PC approximation has been extensively used [1-5] and, for scattering angles largur than 150, has yielded IA differential cross sections in excellent agreement with those calculated in an exact manner, i.e, from the exact expression for the IA collision amplitude, eq. (A24), while consuming a significantly smaller amount of computing time. The PC approximation can thus be used for a more efficient calculation of the quantitics $F_{M_{1}}^{(0)}\left(v^{\prime} j^{\prime} p_{3}^{\prime} ; v_{j} p_{3}\right)$.

The expression for $F_{j 0}^{(\omega) P C}\left(v^{\prime} j^{\prime} p_{3}^{\prime} ; v_{j} p_{3} ; \hat{\mathbf{z}}=\hat{\mathbf{q}}\right)$ was given in SBS. We note that $\hat{\mathbf{z}}=\hat{\mathbf{q}}$ is specified here because this choice of axis is esscntial in the formulation of the PC approximation; this choice also leads to only the quantities with $M_{J}=0$ having nonzero values. From SBS, we have

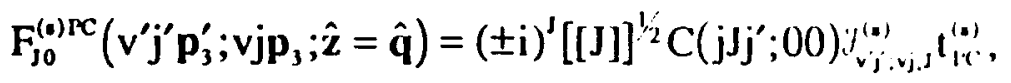

where,

$\underset{v^{\prime} ;: v j: J}{g^{(0)}} \equiv \int_{0}^{\bar{d}} d y_{3} y_{3}^{2} \chi_{v^{\prime j}}\left(y_{3}\right) j_{j}\left(\left|\alpha_{4}\right| q y_{3}\right) \chi_{v j}\left(y_{3}\right)$

In order to apply eq. (A27), it is necessary to obtain the quantities $F_{\mathrm{JM}}^{(0) P C}\left(v^{\prime} \mathrm{j}^{\prime} \mathbf{p}_{3}^{\prime} ; \mathrm{vjp}_{3} ; \hat{\mathbf{z}}=\hat{\mathbf{p}}_{3}\right)$, where the z-axis of the coordinate system is taken along the direction of $\mathbf{p}_{3}$. Taking the vectors $\mathbf{p}_{3}$ and $\mathbf{p}_{3}^{\prime}$ in the $x-z$ plane, these quantities can be obtained from the $F_{j 0}^{(0) P C}\left(v^{\prime} j^{\prime} \mathbf{p}_{3}^{\prime} ; v j p_{3} ; \hat{z}=\hat{\mathbf{q}}\right)$ by the simple transformation:

$F_{J M_{1}}^{(o) P C}\left(v^{\prime} j^{\prime} \mathbf{p}_{3}^{\prime} ; v j p_{3} ; \hat{\mathbf{z}}=\hat{\mathbf{p}}_{3}\right)=F_{30}^{(\lrcorner) P C}\left(v^{\prime} j^{\prime} \mathbf{p}_{3}^{\prime} ; v j p_{3} ; \hat{\mathbf{z}}=\hat{\mathbf{q}}\right) D_{u M_{1}}^{\prime}(0, \tau, 0)$,

where $\tau$ is the angle between $p_{3}$ and $q$, and $D_{u M_{1}}^{\prime}(0, \tau, 0)$ is defined by [16]: 
$D_{0 M}^{\prime}(0, \tau, 0)=\left(\frac{4 \pi}{[J]}\right)^{\frac{1}{2}} Y_{1-M_{1}}(\tau, 0)$

From eqs. (A30), (A32) and (A27), the quantities $A_{\mu_{1} f_{i}^{\prime}}^{(s)}\left(v^{\prime} j^{\prime} p_{3}^{\prime} ; v_{j p}\right)$ can be computed in the part-classical approximation. Inserting these in eq. (A21), the IA collision amplitude (part-classical) is obtained. 


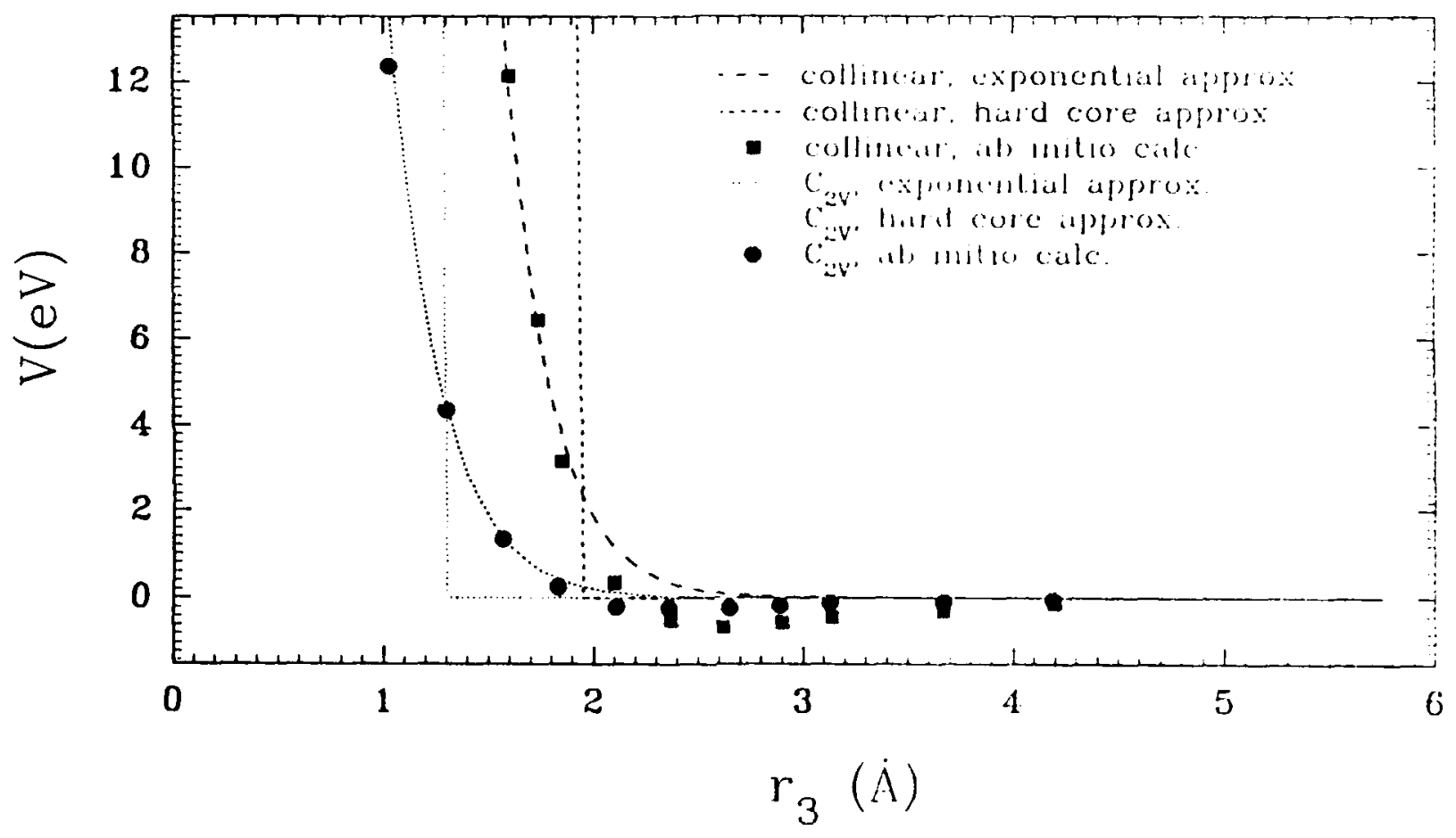

Figure 1 


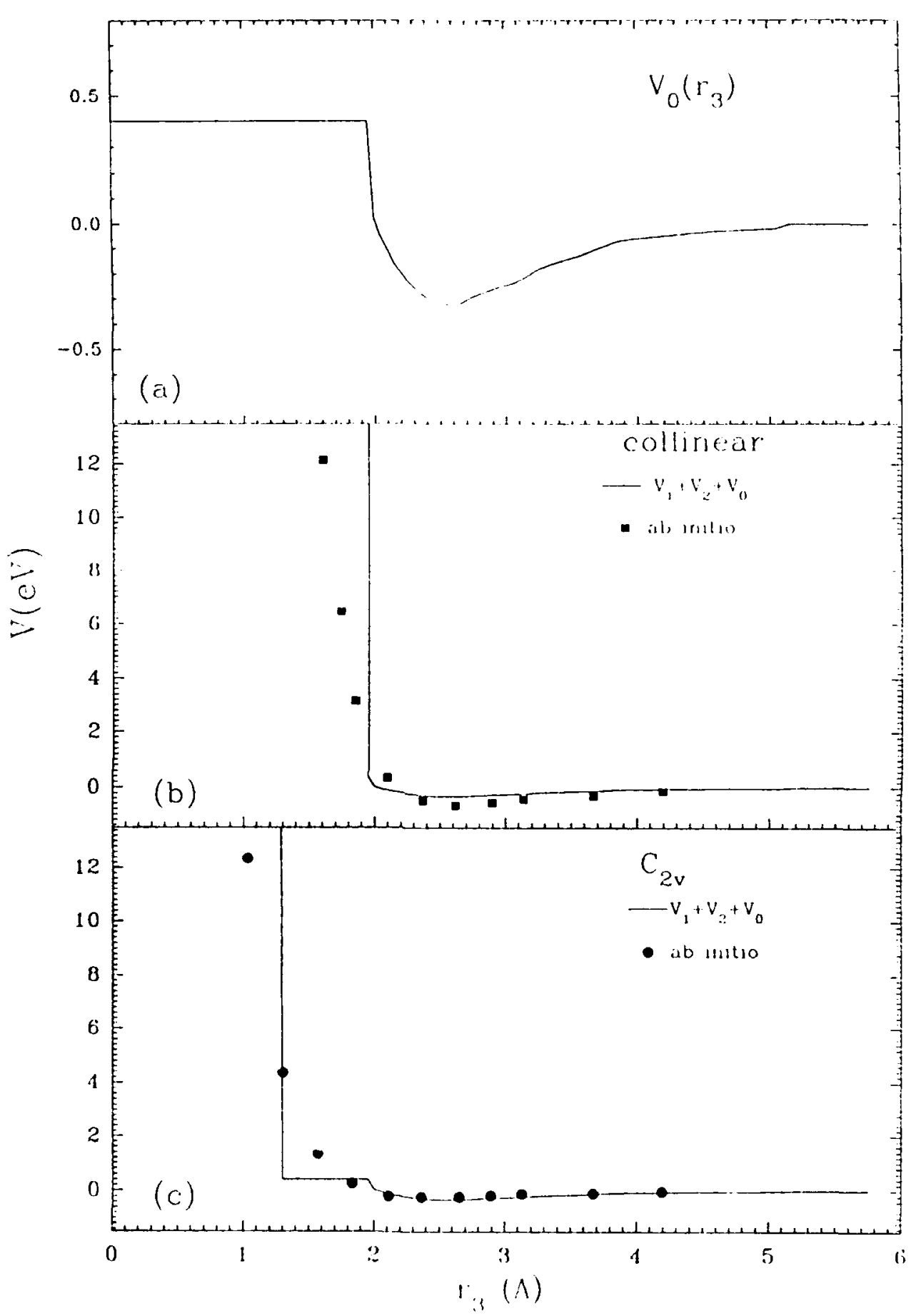

Figure 2 


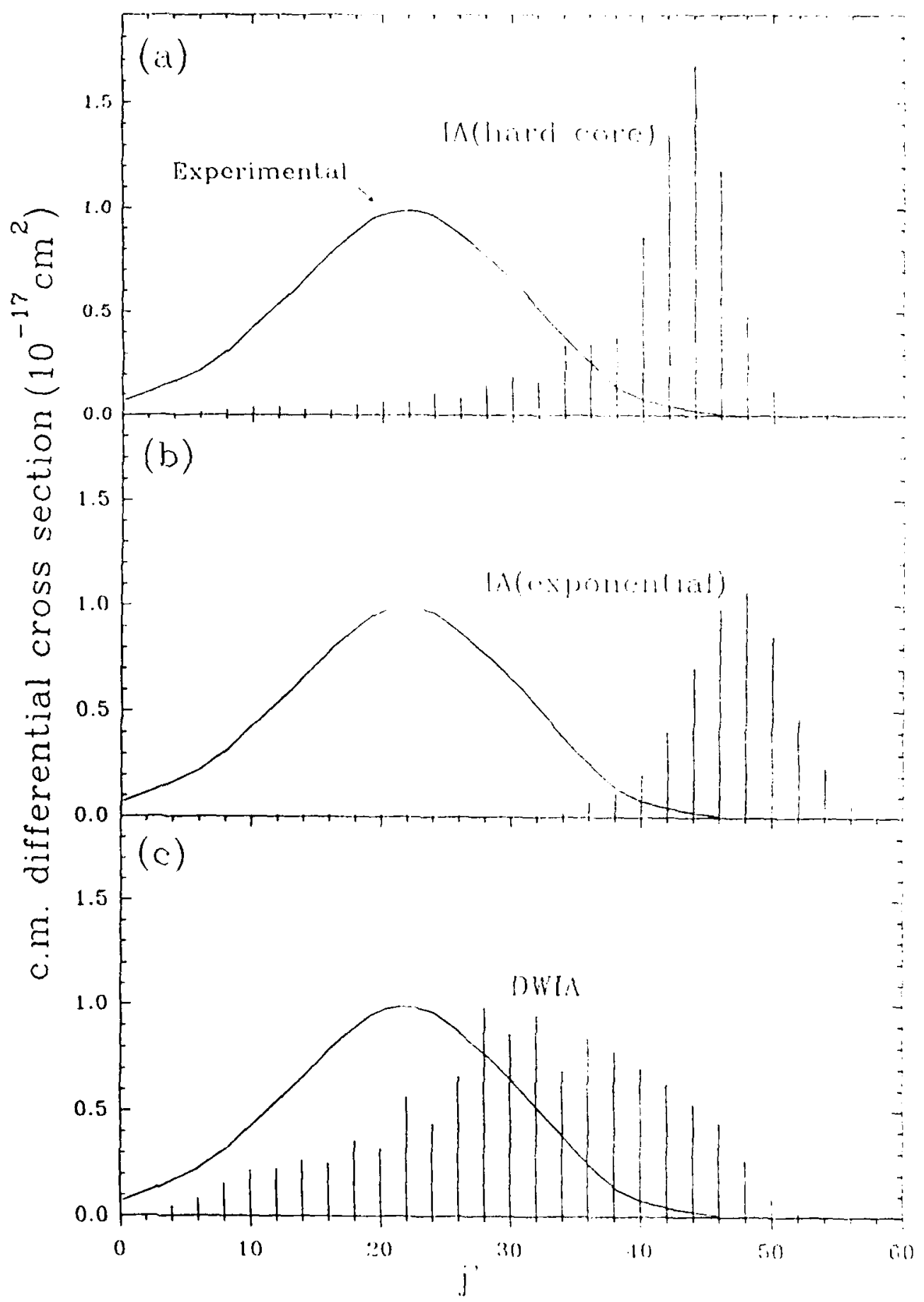

Figure 3 


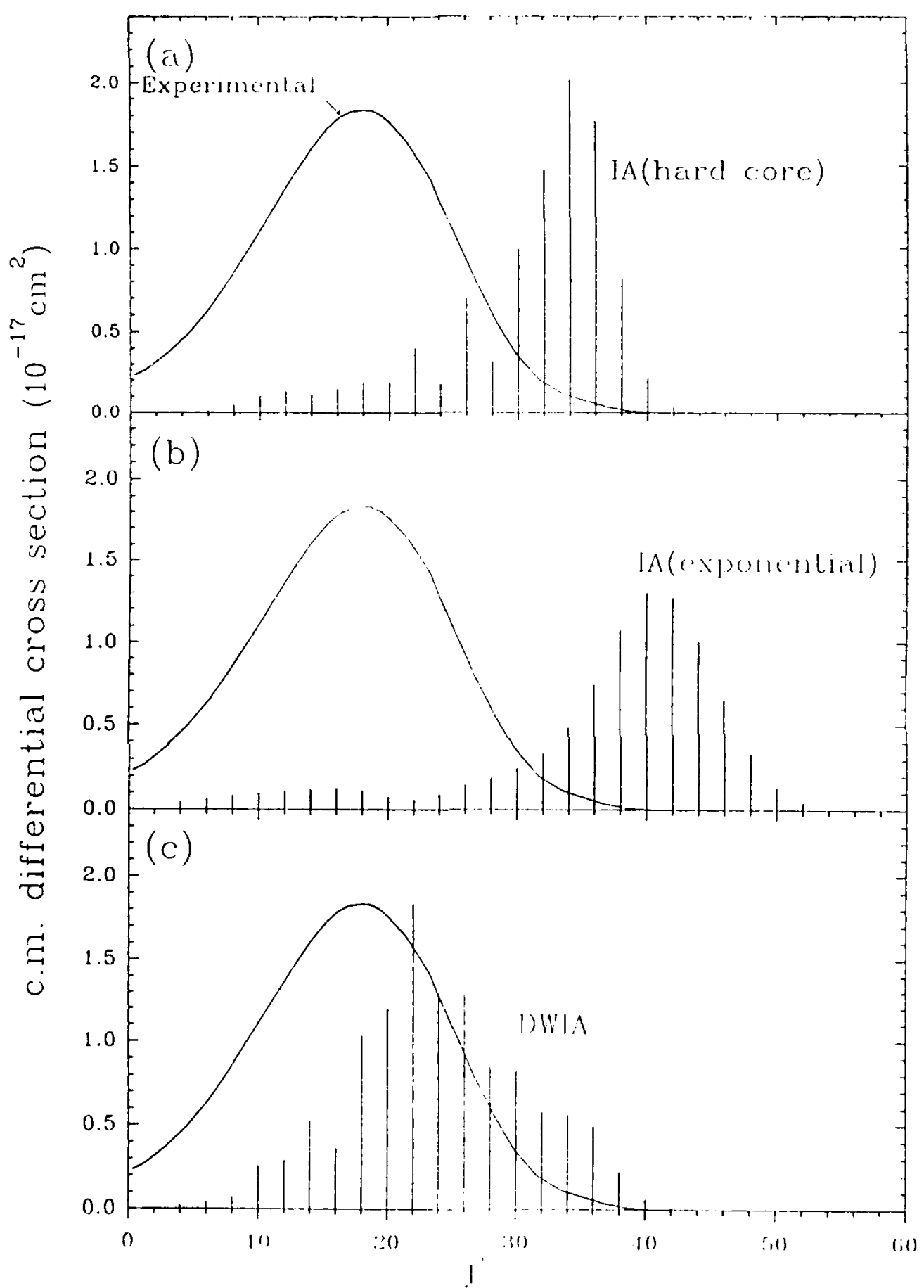

Figure 4 


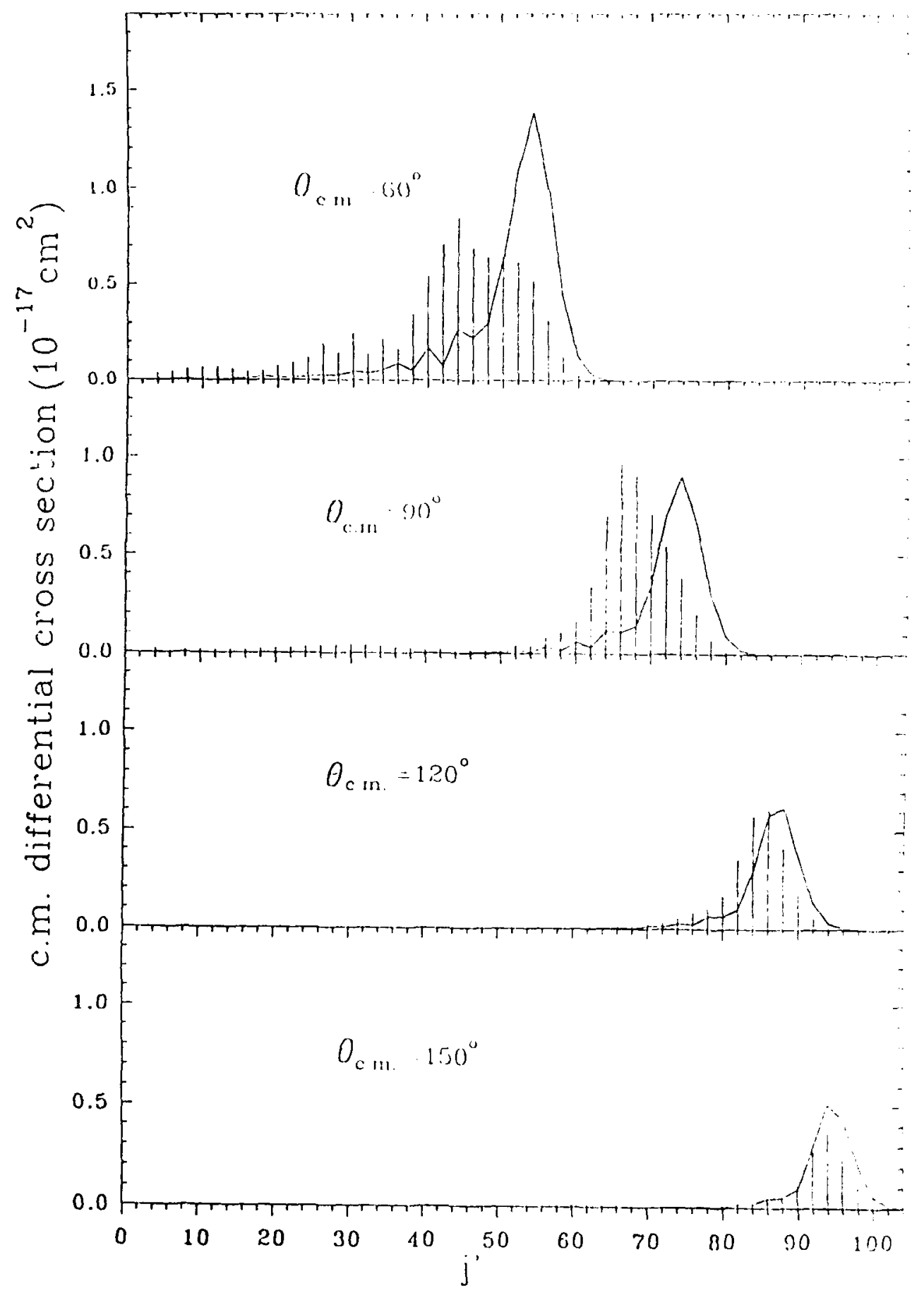

Figure 5 
Table 1.

Ratio of part-classical and exact DWIA differential cross sections for the inelastic process $\mathrm{Li}^{+}+\mathrm{N}_{2}(\mathrm{v}=0, \mathrm{j}=2) \rightarrow \mathrm{Li}^{+}+\mathrm{N}_{2}\left(\mathrm{v}^{\prime}=0, \mathrm{j}^{\prime}\right)$ at a relative kinetic energy of $4.23 \mathrm{eV}$

$\begin{array}{ccc}j^{\prime} & \theta_{\text {c.m. }}=37.1^{0} & \theta_{\mathrm{c} . \mathrm{m} .}=49.1^{\mathrm{O}} \\ 4 & 14.24 & 2.39 \\ 6 & 9.08 & 1.38 \\ 8 & 4.02 & 1.36 \\ 10 & 1.53 & 1.09 \\ 12 & 1.03 & 1.08 \\ 14 & 1.02 & 0.98 \\ 16 & 0.99 & 1.00 \\ 18 & 1.00 & 1.00 \\ 20 & 1.00 & 1.00 \\ 22 & 1.00 & 1.00 \\ 24 & 1.00 & 1.00 \\ 26 & 1.00 & 1.00 \\ 28 & 1.00 & 1.00 \\ 30 & 1.00 & 1.00 \\ 32 & 1.00 & 1.00 \\ 34 & 1.00 & 1.00 \\ 36 & 1.00 & 1.00 \\ 38 & 1.00 & 1.00 \\ 40 & 1.00 & 1.00 \\ 42 & 1.00 & 1.00 \\ 44 & & 1.12 \\ 46 & & 1.14 \\ 48 & & 1.00 \\ 50 & & 1.00\end{array}$

